\title{
A SYNTHETIC ROUTE TO PHENYL DIAZENES AND PYRIDAZINIUM SALTS FROM PHENYLAZOSULFONATES
}

Susanne Gradl, Johannes Köckenberger, Janina Oppl, Martin Schiller, Markus R. Heinrich* Department of Chemistry and Pharmacy, Pharmaceutical Chemistry, Friedrich-Alexander-Universität ErlangenNürnberg, Nikolaus-Fiebiger-Str. 10, 91058 Erlangen, Germany.

Supporting Information

Table of Contents

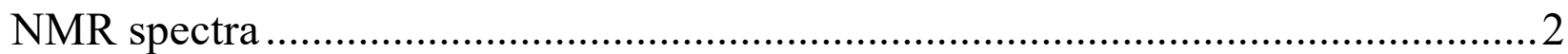

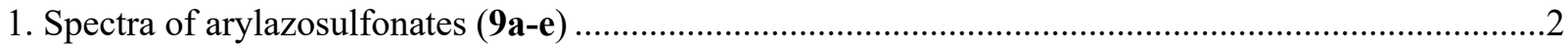

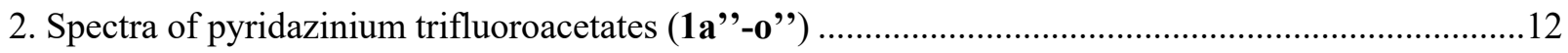

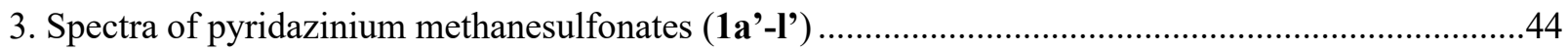

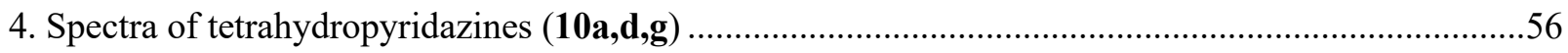

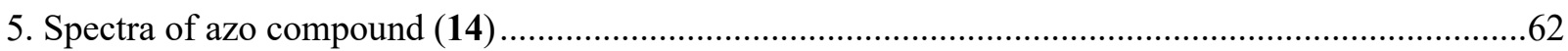




\section{NMR spectra}

\section{Spectra of arylazosulfonates (9a-e)}

Sodium 2-(4-chlorophenyl)diazene-1-sulfonate (9a) ${ }^{1}$ H-NMR (DMSO-d 6 , 400 MHz)
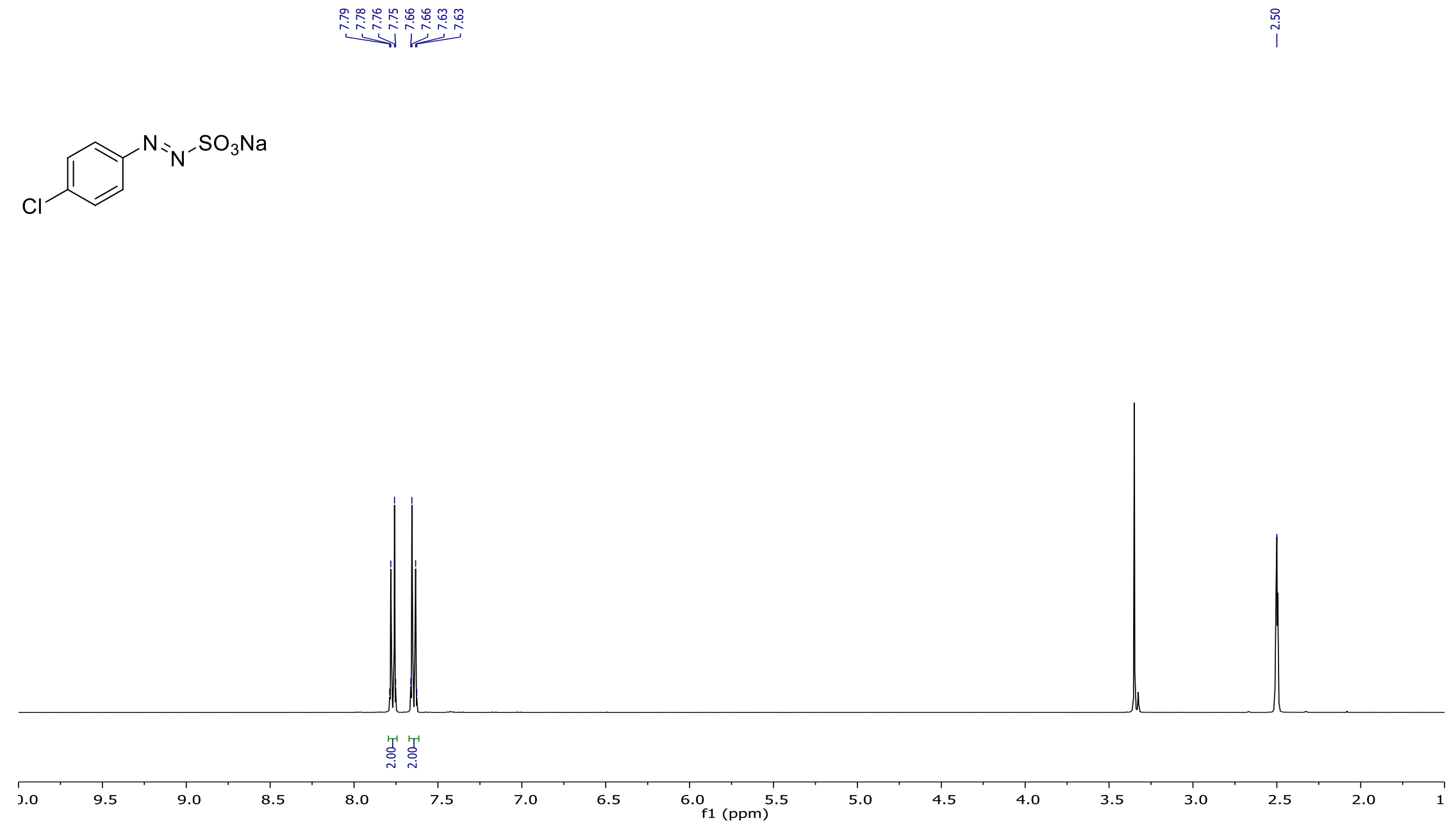
Sodium 2-(4-chlorophenyl)diazene-1-sulfonate (9a) DEPTQ-NMR (DMSO-d 6 , 100 MHz)
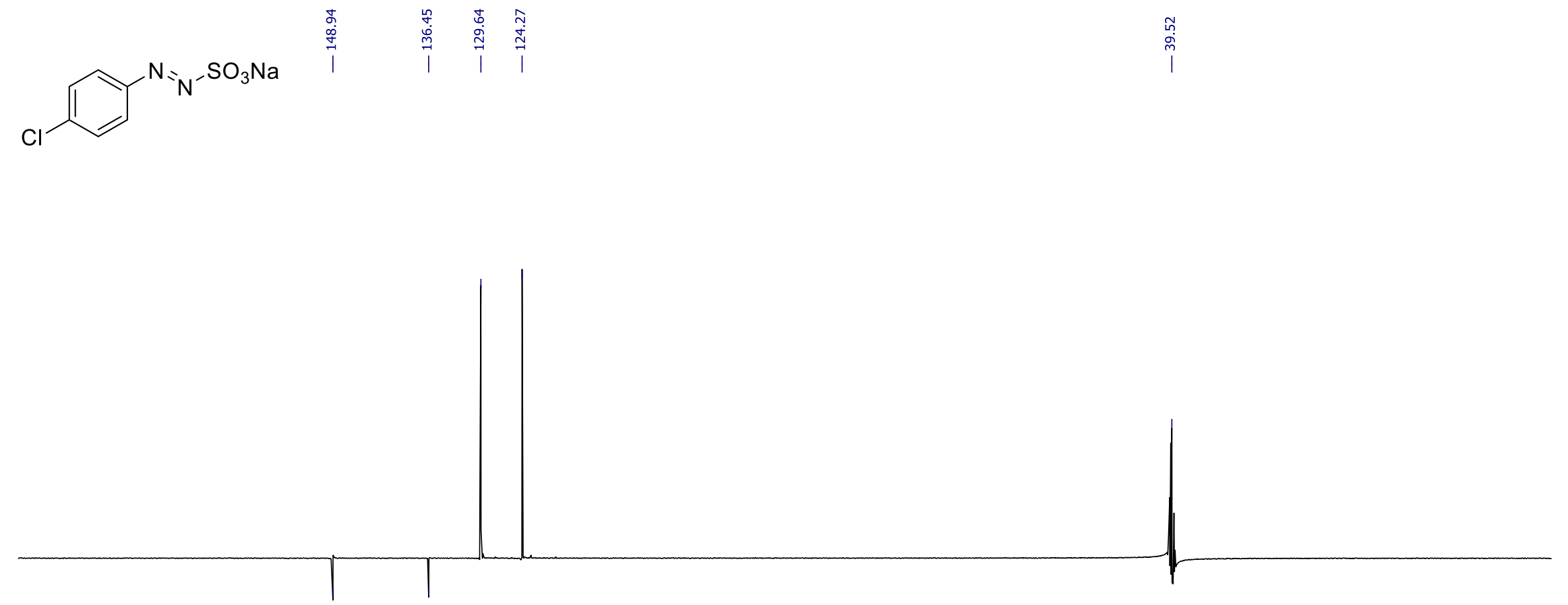

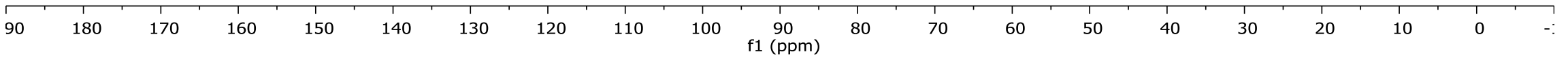


Sodium 2-(4-fluorophenyl)diazene-1-sulfonate (9b) ${ }^{1}$ H-NMR (DMSO-d ${ }_{6}, 400 \mathrm{MHz}$ )
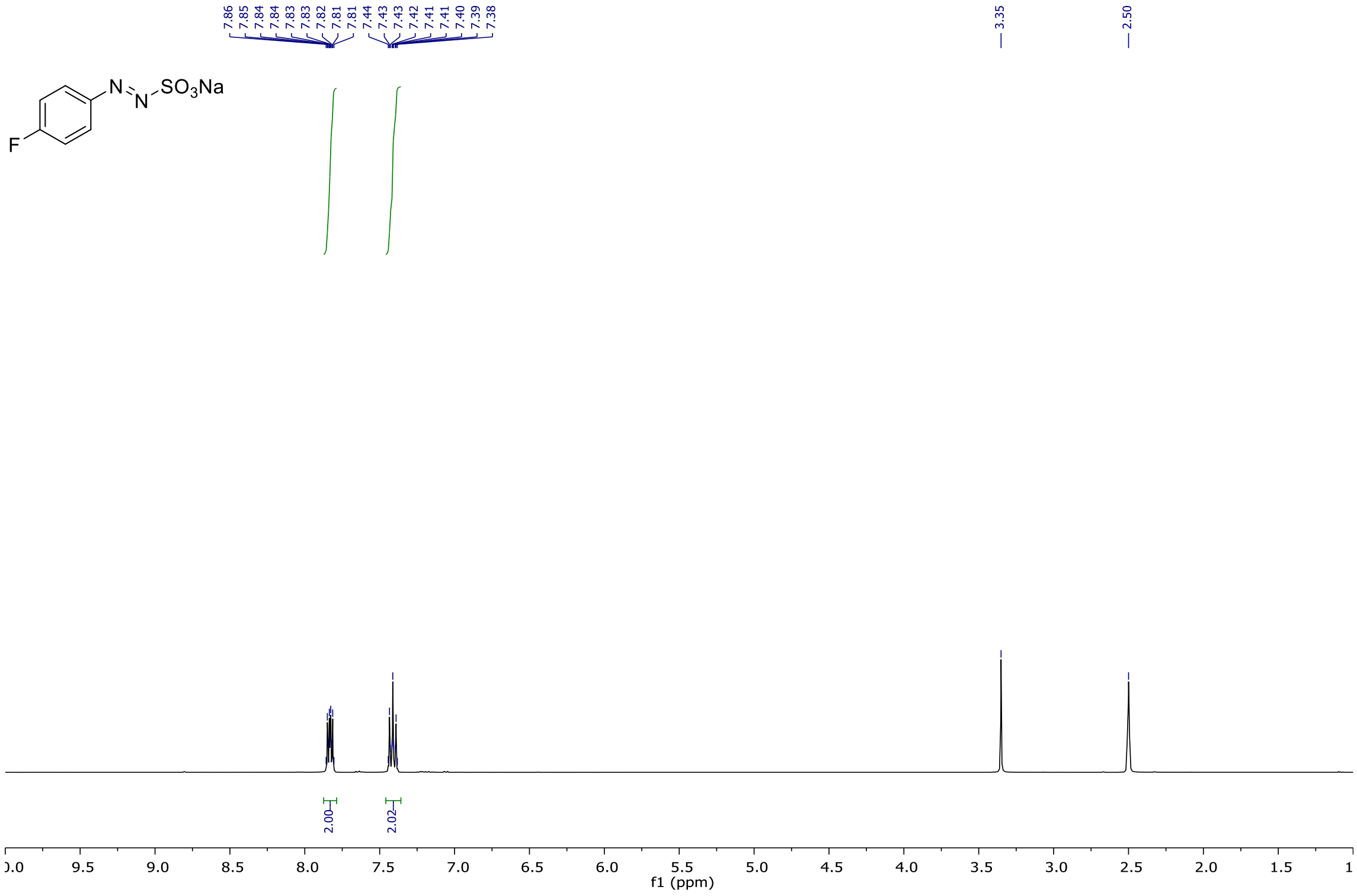
Sodium 2-(4-fluorophenyl)diazene-1-sulfonate (9b) DEPTQ-NMR (DMSO-d 6 , 151 MHz)

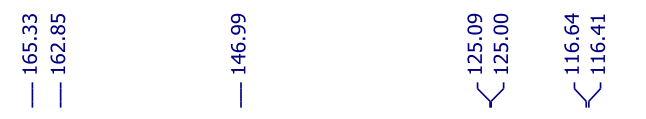
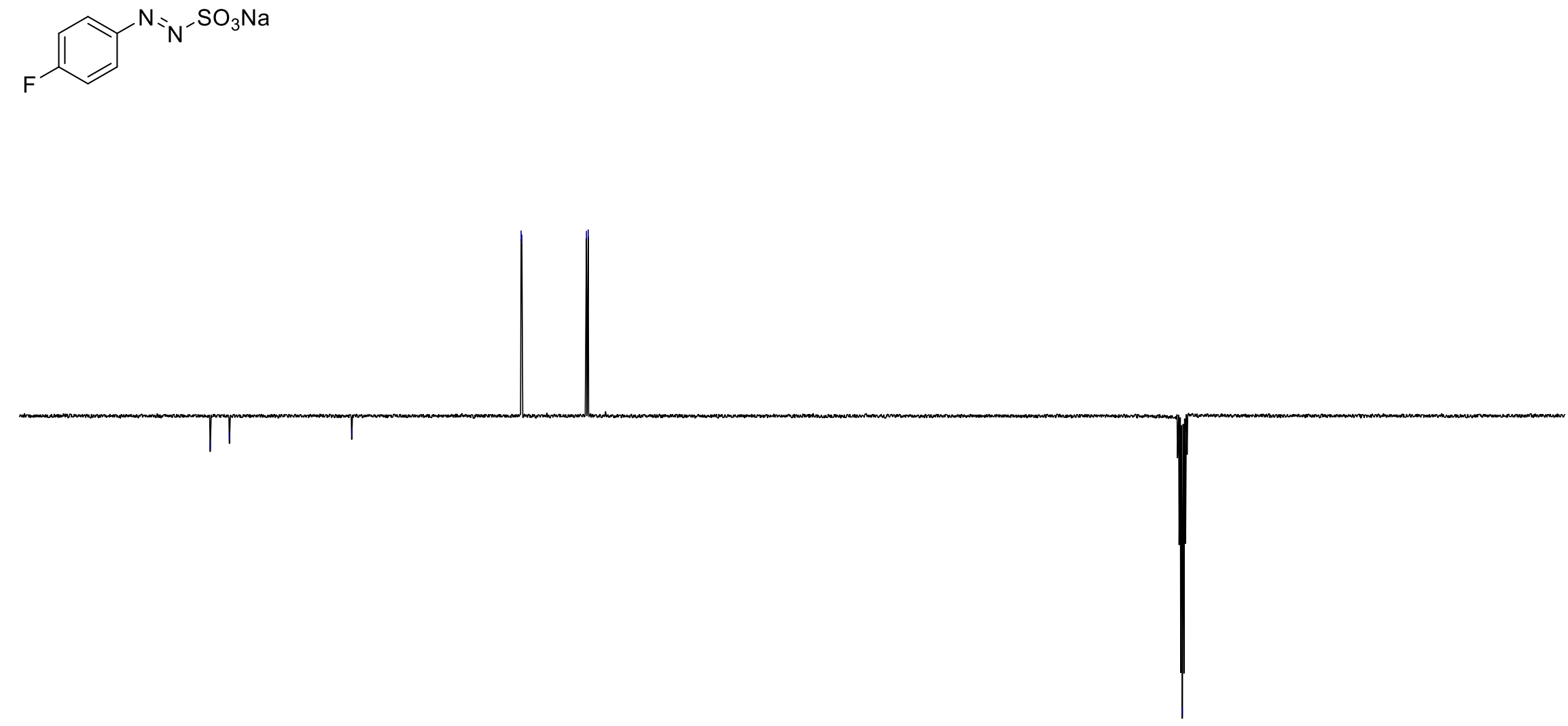
Sodium 2-(4-methoxyphenyl)diazene-1-sulfonate (9c) ${ }^{1} \mathrm{H}-\mathrm{NMR}$ (DMSO-d ${ }_{6}, 400 \mathrm{MHz}$ )
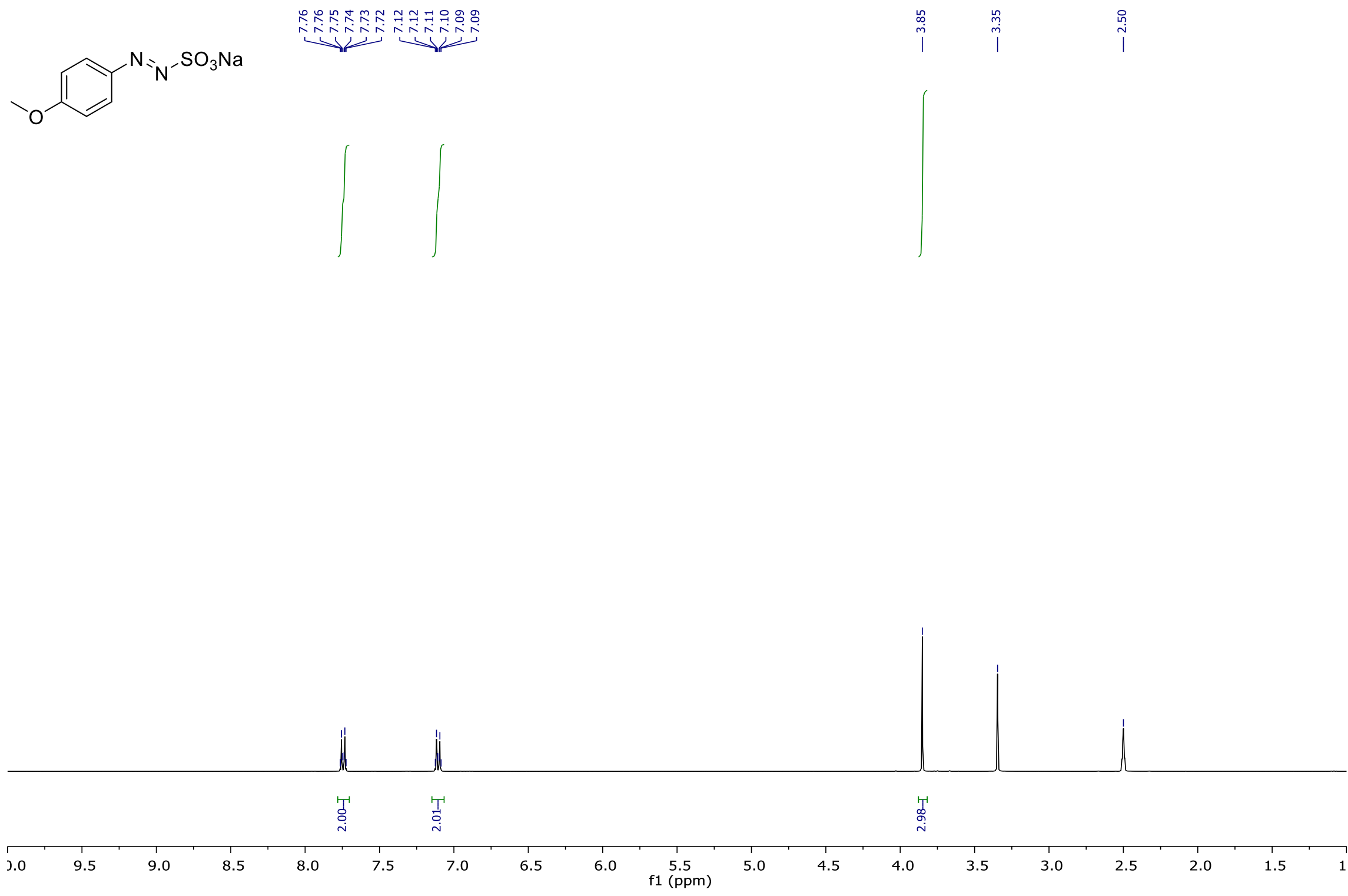
Sodium 2-(4-methoxyphenyl)diazene-1-sulfonate (9c) DEPTQ-NMR (DMSO-d 6 , $100 \mathrm{MHz})$

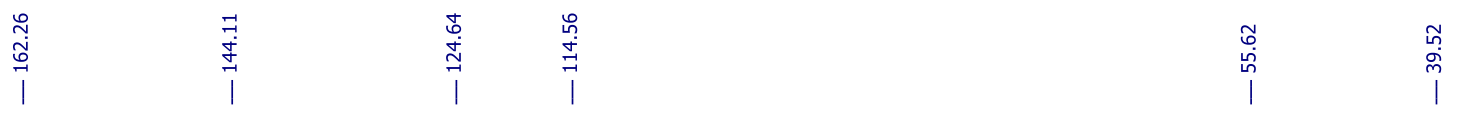

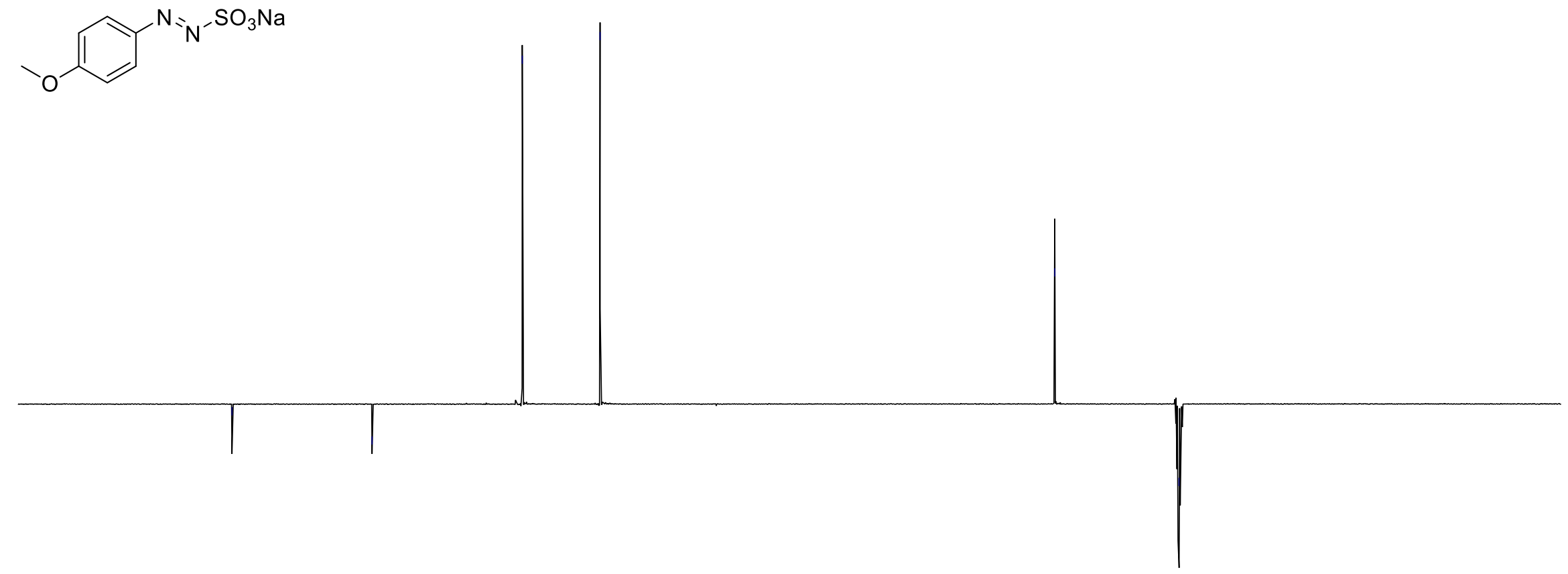


Sodium 2-(4-cyanophenyl)diazene-1-sulfonate (9d) ${ }^{1} \mathrm{H}-\mathrm{NMR}$ (DMSO-d 6 , $\left.400 \mathrm{MHz}\right)$
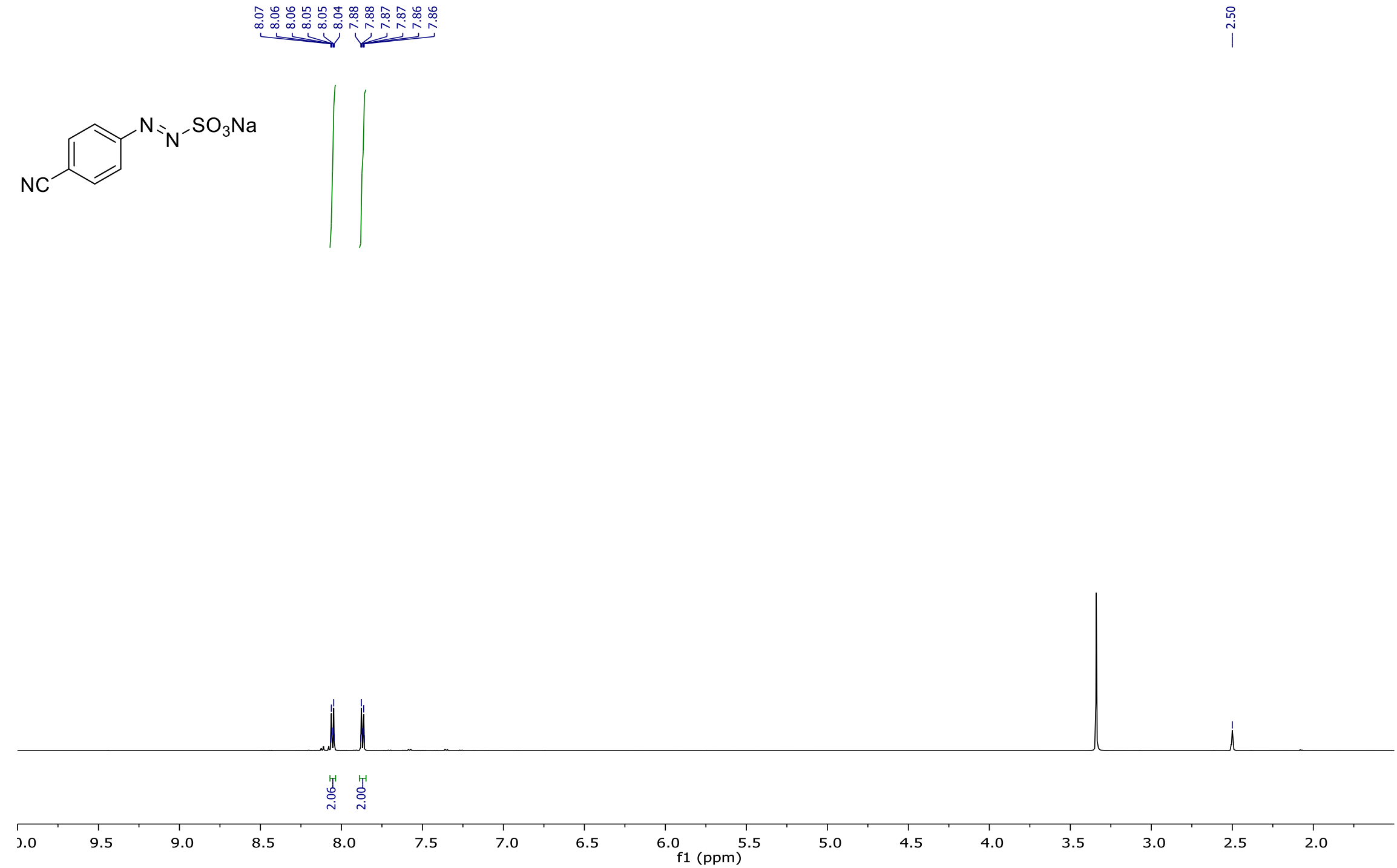
Sodium 2-(4-cyanophenyl)diazene-1-sulfonate (9d) DEPTQ-NMR (DMSO-d 6 , 100 MHz)

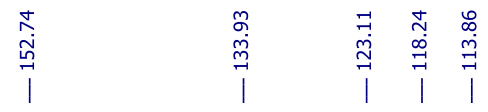

$\mathrm{N}=\mathrm{N}^{-} \mathrm{SO}_{3} \mathrm{Na}$

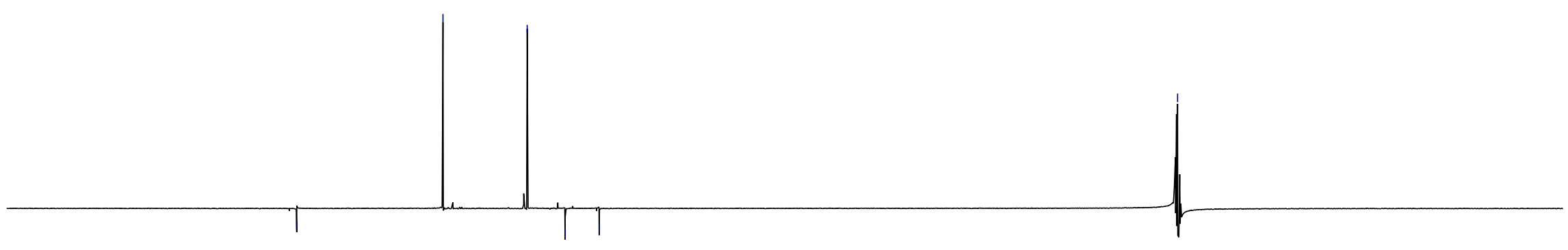

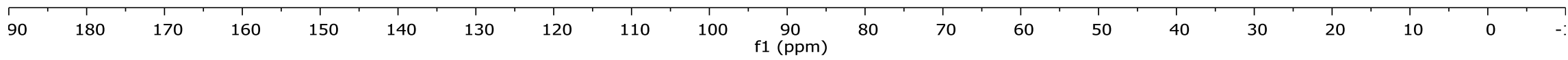


Sodium 2-phenyldiazene-1-sulfonate (9e) ${ }^{1} \mathrm{H}-\mathrm{NMR}$ (DMSO-d 6 , $400 \mathrm{MHz}$ )

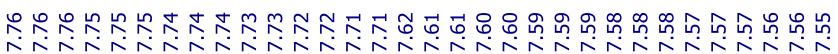
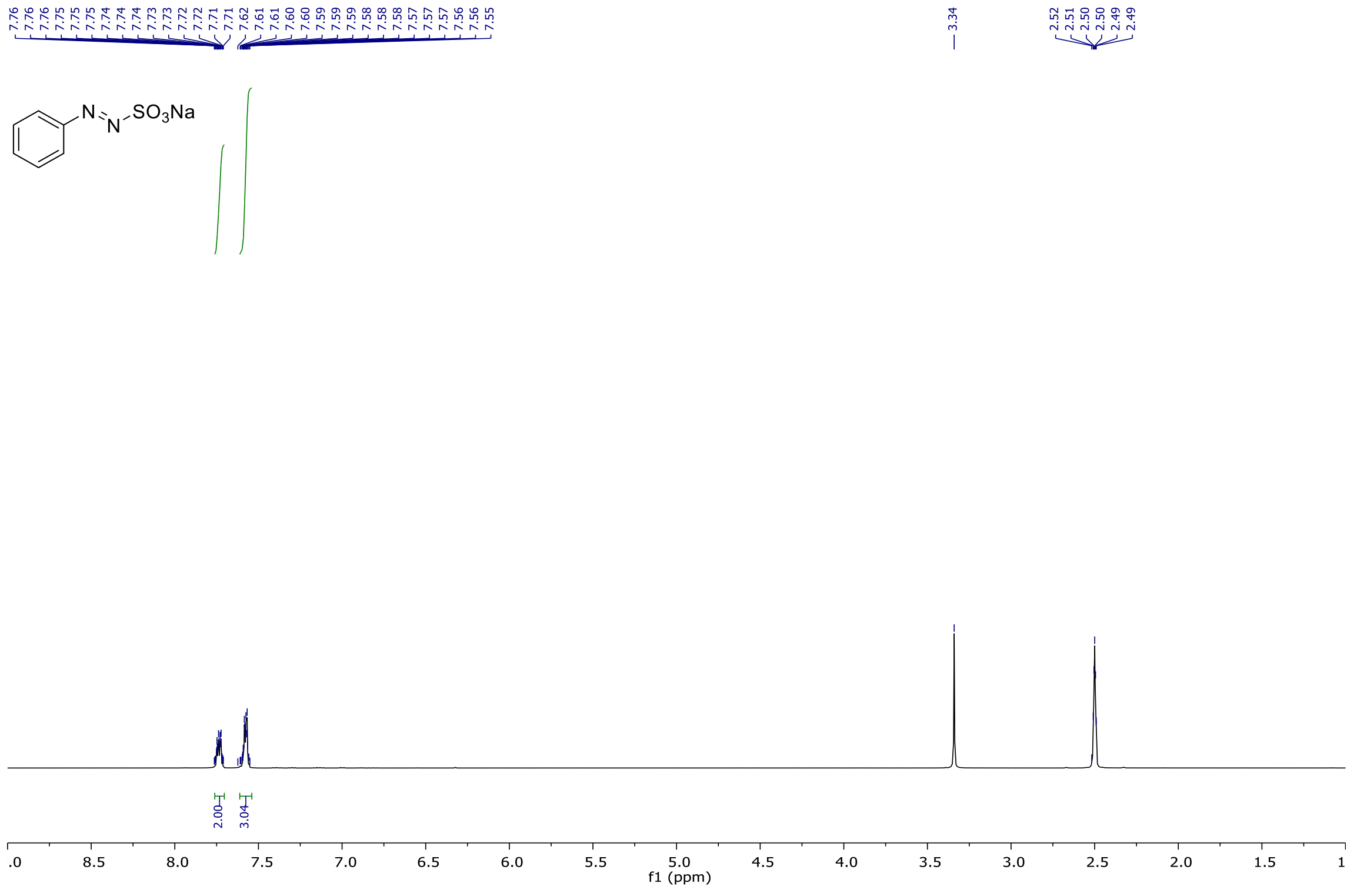
Sodium 2-phenyldiazene-1-sulfonate (9e) DEPTQ-NMR (DMSO-d 6 , 100 MHz)

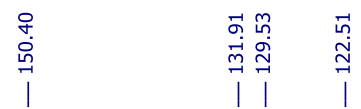
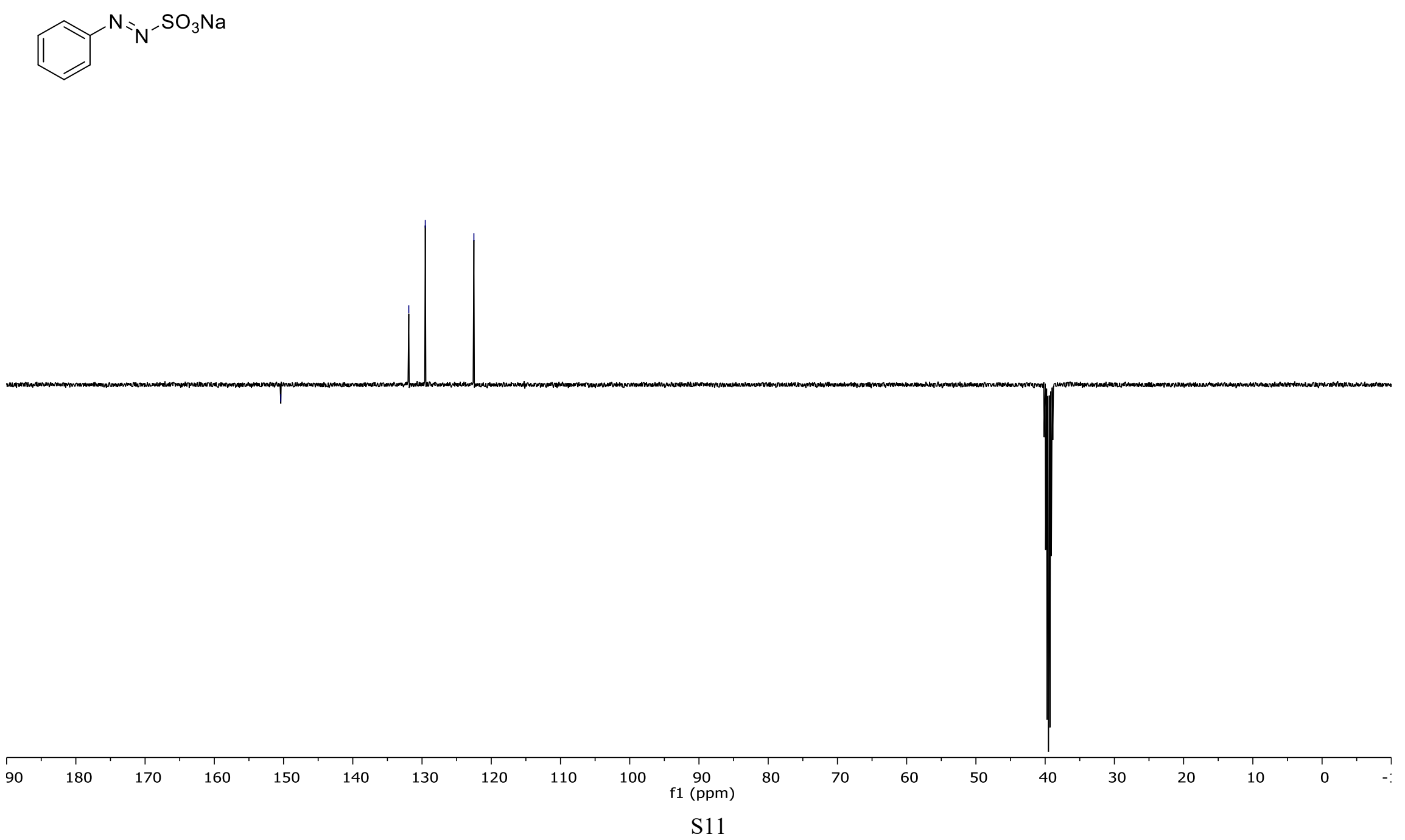
2. Spectra of pyridazinium trifluoroacetates (1a'-o')

1-(4-Chlorophenyl)-3,6-dimethylpyridazin-1-ium trifluoroacetate (1a'") ${ }^{1} \mathrm{H}-\mathrm{NMR}\left(\mathrm{CD}_{3} \mathrm{CN}, 400 \mathrm{MHz}\right)$
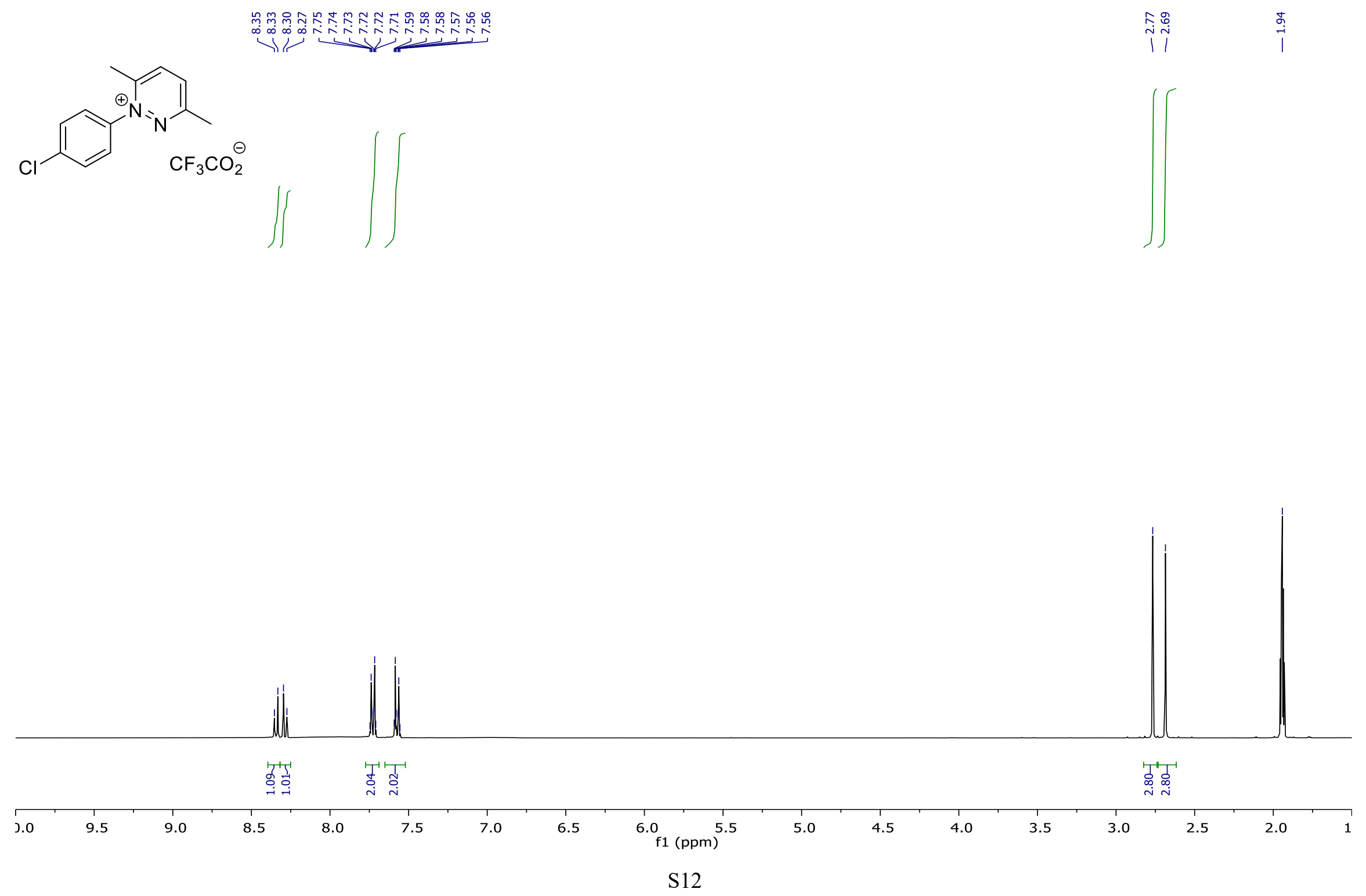
1-(4-Chlorophenyl)-3,6-dimethylpyridazin-1-ium trifluoroacetate (1a'”) DEPTQ-NMR $\left(\mathrm{CD}_{3} \mathrm{CN}, 151 \mathrm{MHz}\right)$

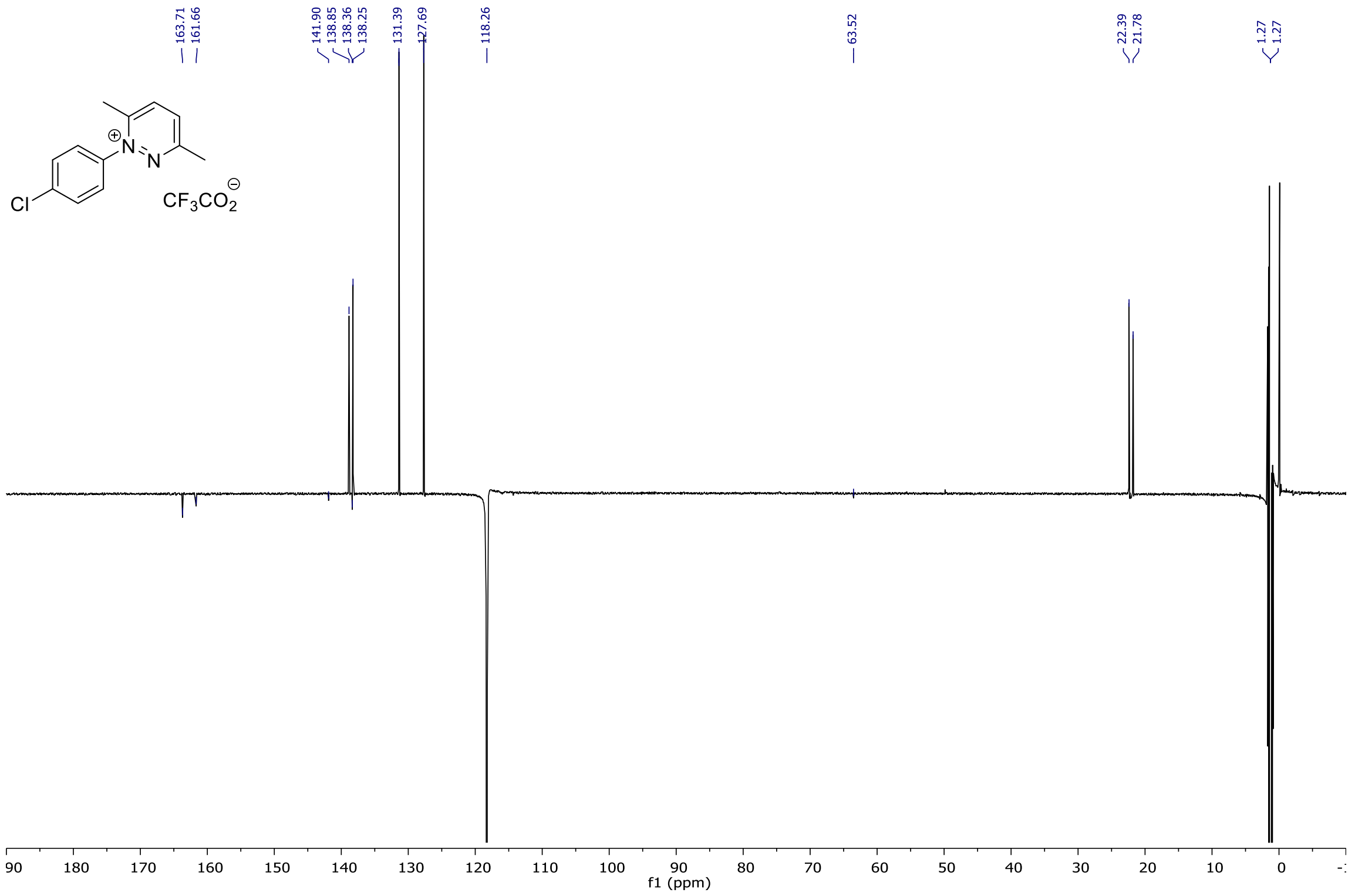


1-(4-Chlorophenyl)-6-methylpyridazin-1-ium trifluoroacetate (1b') ${ }^{1} \mathrm{H}-\mathrm{NMR}\left(\mathrm{CD}_{3} \mathrm{CN}, 400 \mathrm{MHz}\right)$

$\underbrace{\infty}$

$\int \mid$
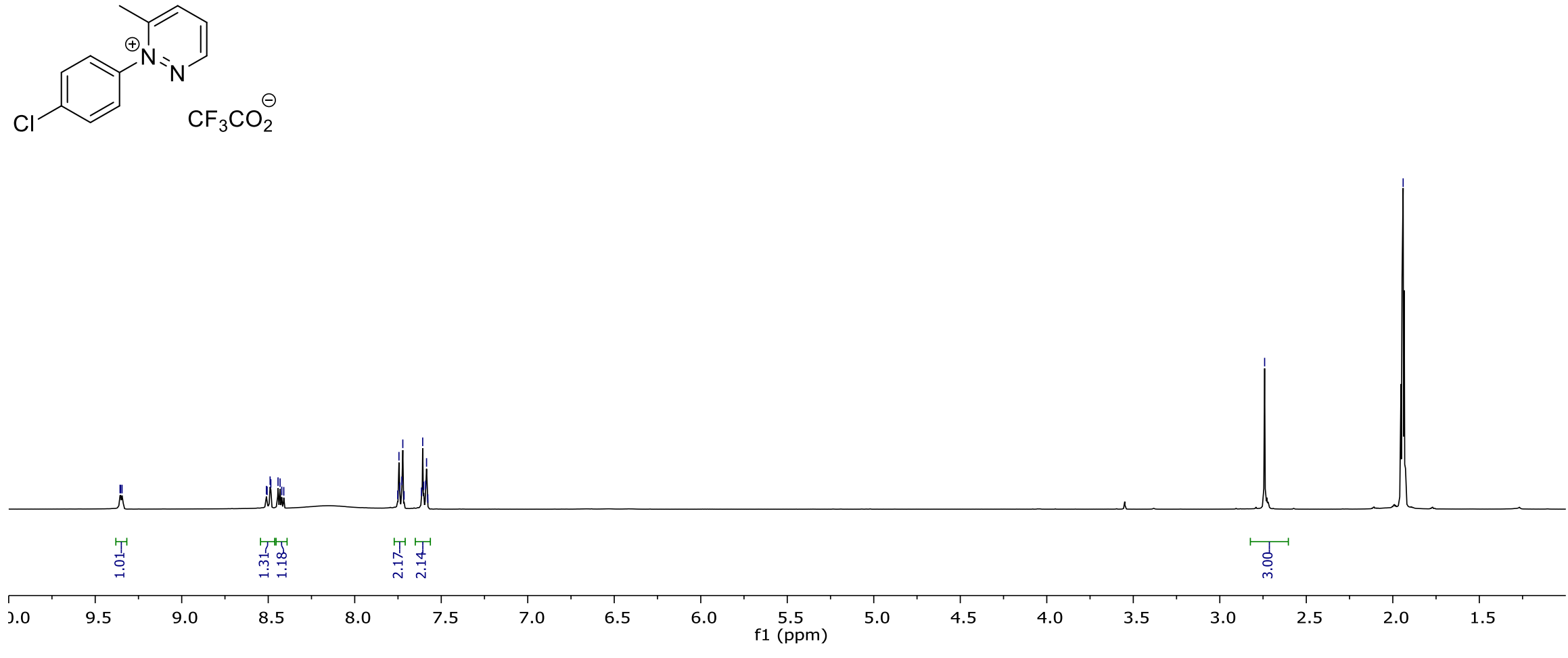
1-(4-Chlorophenyl)-6-methylpyridazin-1-ium trifluoroacetate (1b') DEPTQ-NMR (CD $\left.{ }_{3} \mathrm{CN}, 151 \mathrm{MHz}\right)$

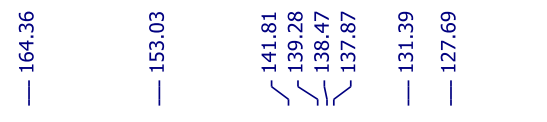
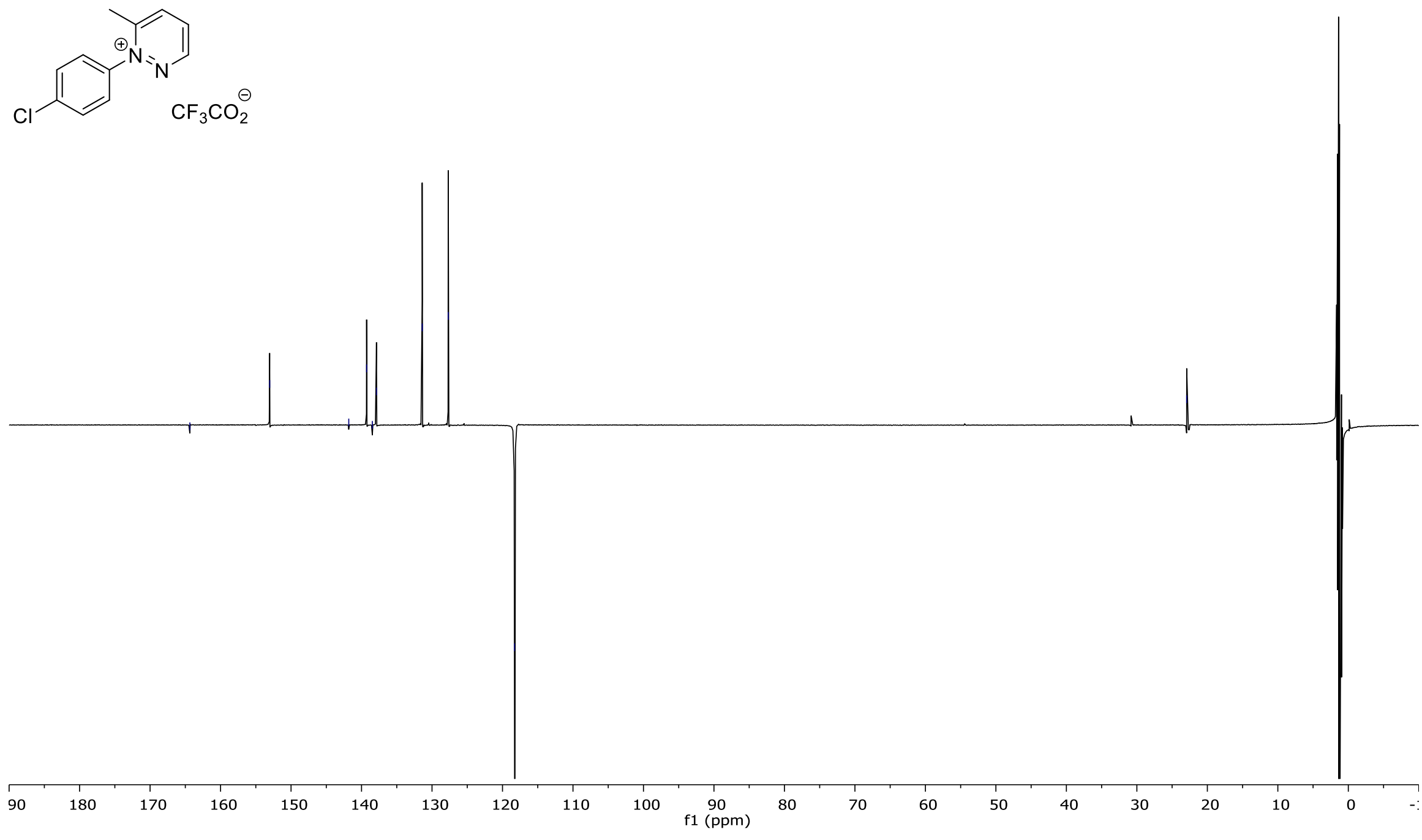
6-(Acetamidomethyl)-1-(4-chlorophenyl)pyridazin-1-ium trifluoroacetate (1c") ${ }^{1} \mathrm{H}-\mathrm{NMR}\left(\mathrm{CD}_{3} \mathrm{CN}, 400 \mathrm{MHz}\right)$

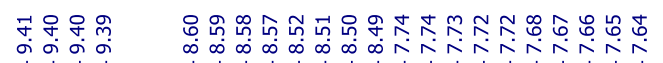

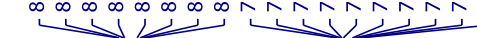

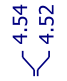

$\int \|$

(N)$$
\mathrm{CF}_{3} \mathrm{CO}_{2}
$$

$$
\text { (1) }
$$
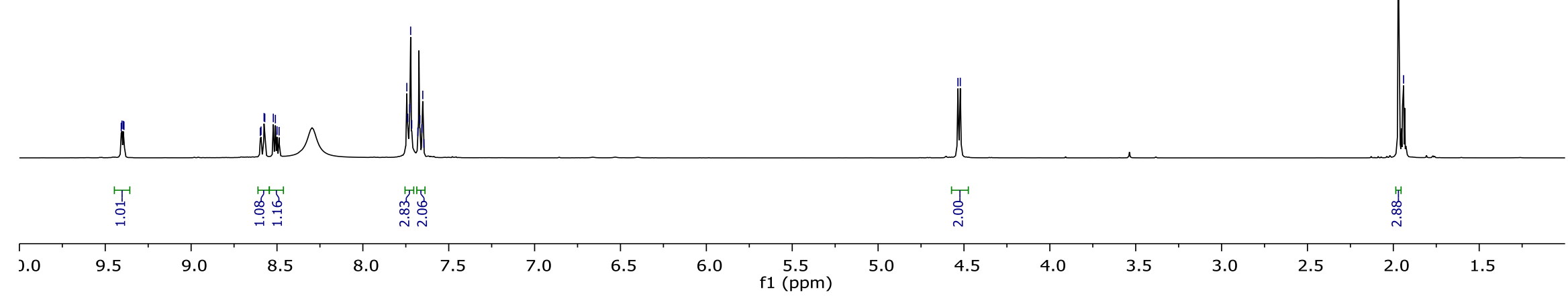
6-(Acetamidomethyl)-1-(4-chlorophenyl)pyridazin-1-ium trifluoroacetate (1c") DEPTQ-NMR (CD 3 CN, $151 \mathrm{MHz})$
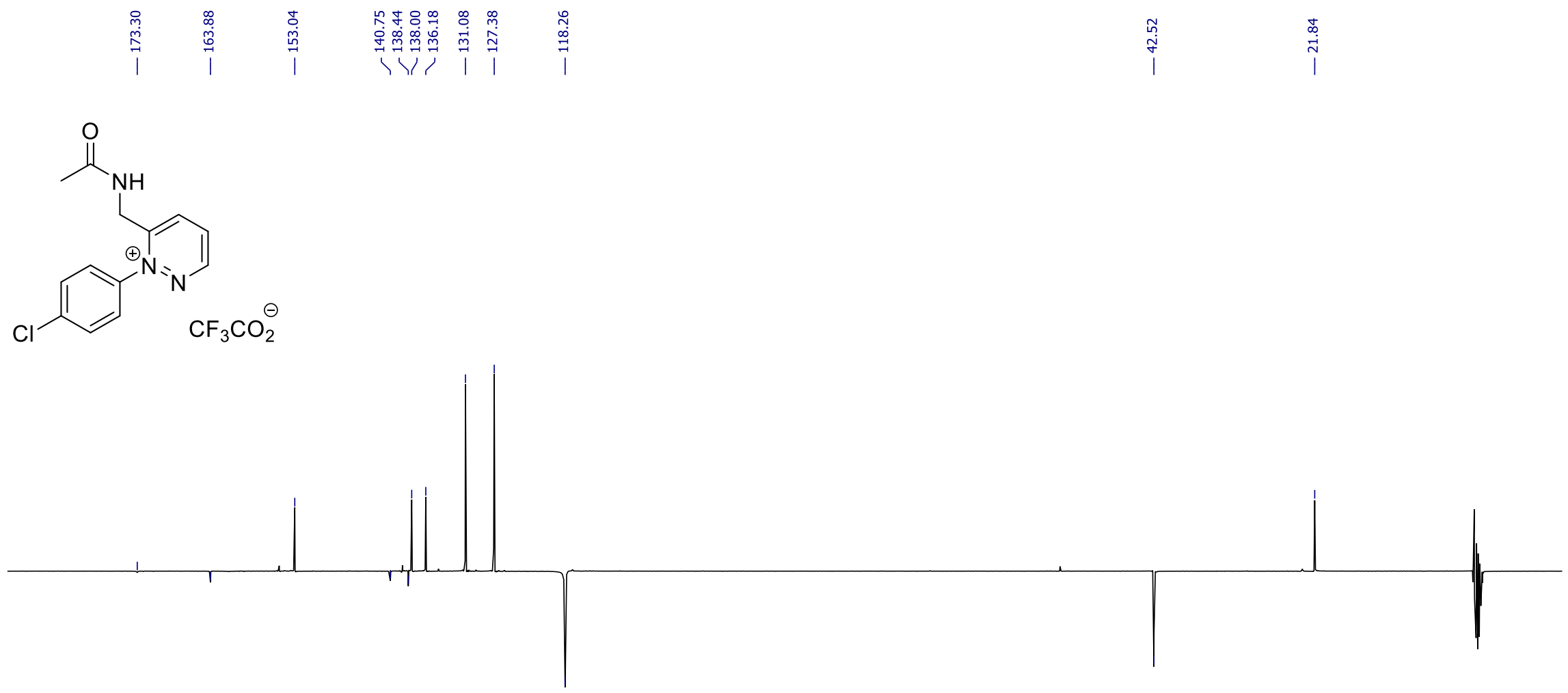

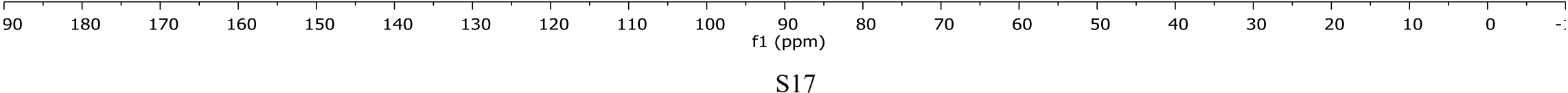


1-(4-Fluorophenyl)-3,6-dimethylpyridazin-1-ium trifluoroacetate (1d"') ${ }^{1} \mathrm{H}-\mathrm{NMR}\left(\mathrm{CD}_{3} \mathrm{CN}, 400 \mathrm{MHz}\right)$

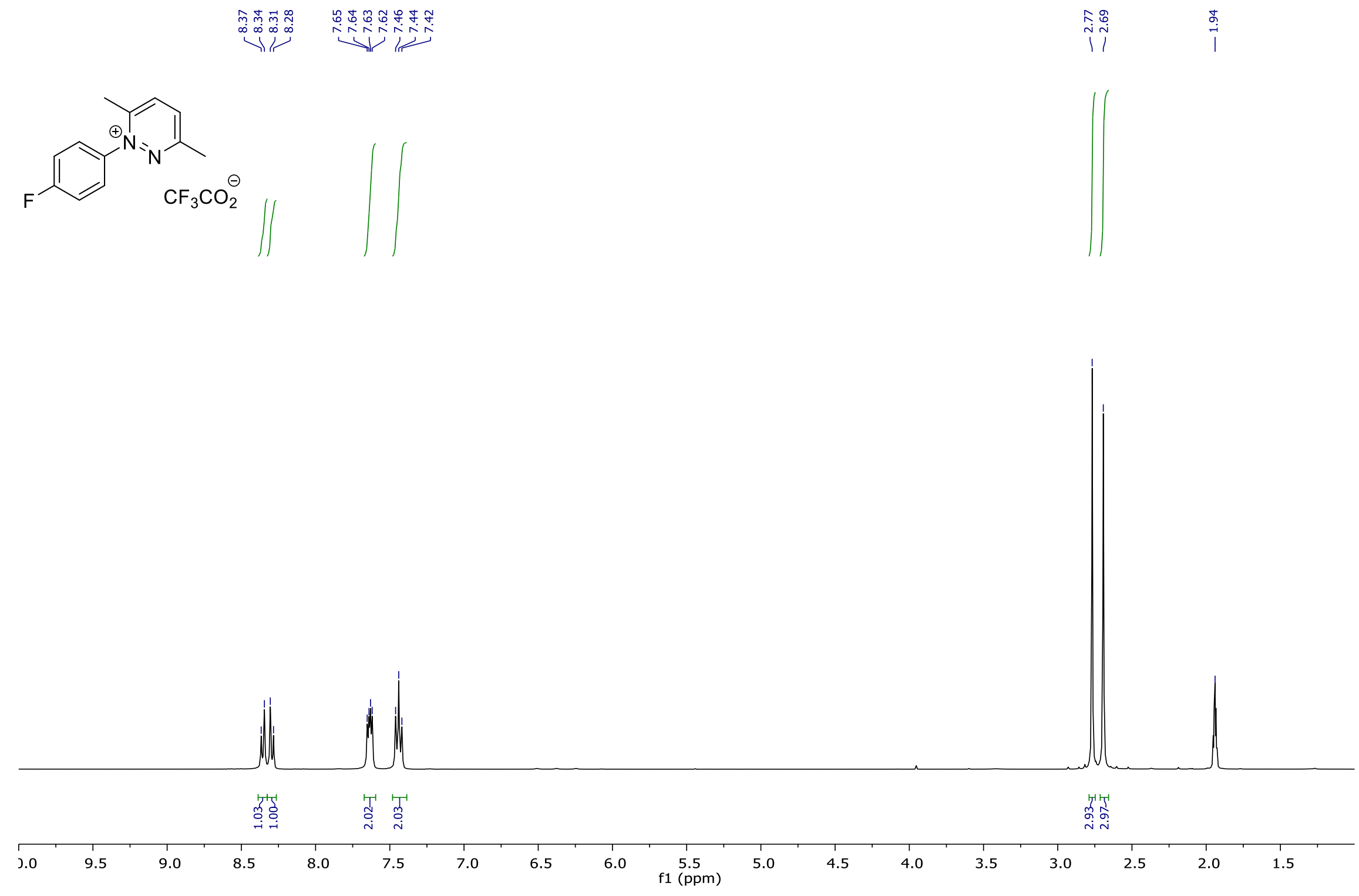


1-(4-Fluorophenyl)-3,6-dimethylpyridazin-1-ium trifluoroacetate (1d") DEPTQ-NMR (CD 3 CN, $151 \mathrm{MHz})$

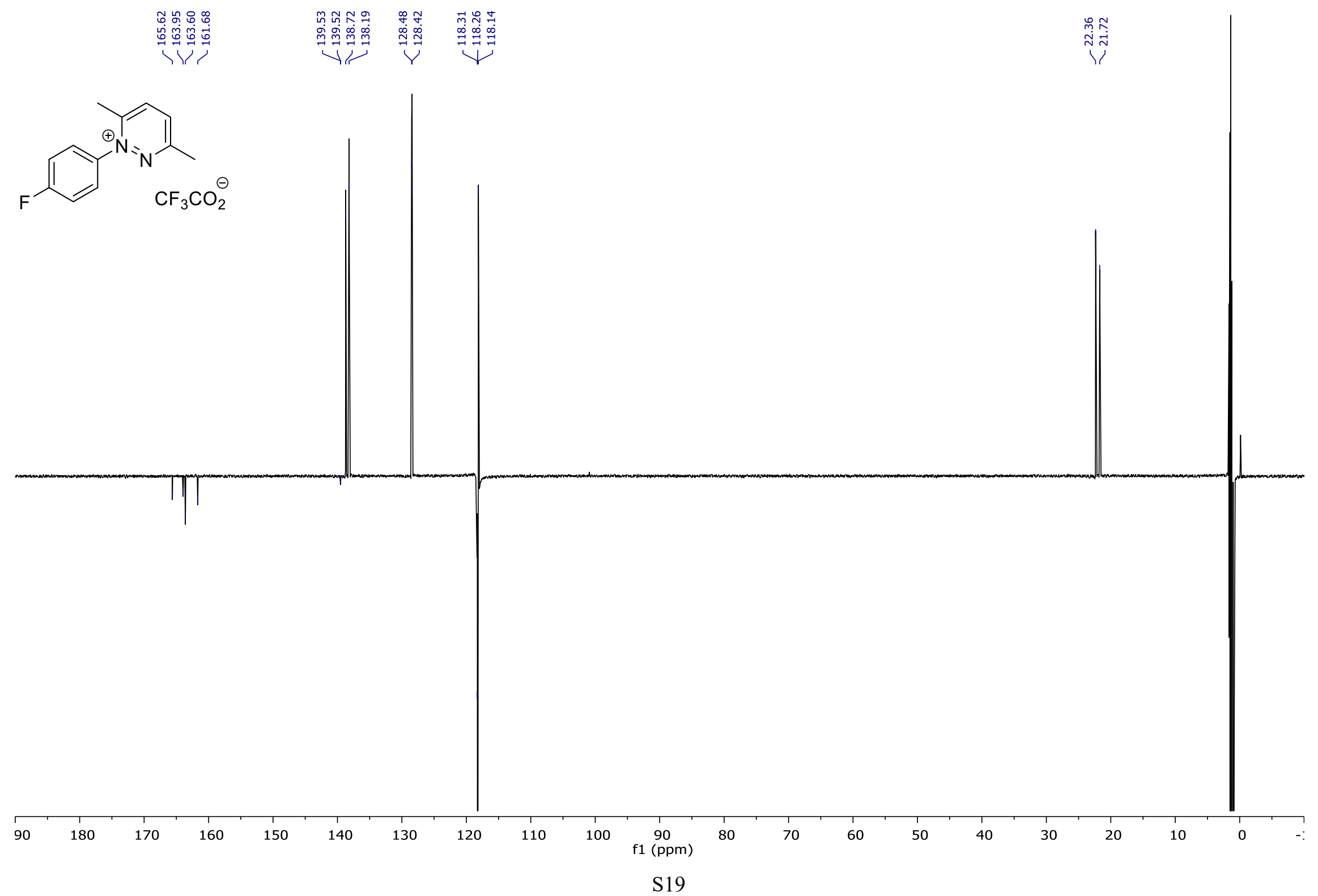


1-(4-Fluorophenyl)-6-methylpyridazin-1-ium trifluoroacetate (1e") ${ }^{1} \mathrm{H}-\mathrm{NMR}\left(\mathrm{CD}_{3} \mathrm{CN}, 400 \mathrm{MHz}\right)$
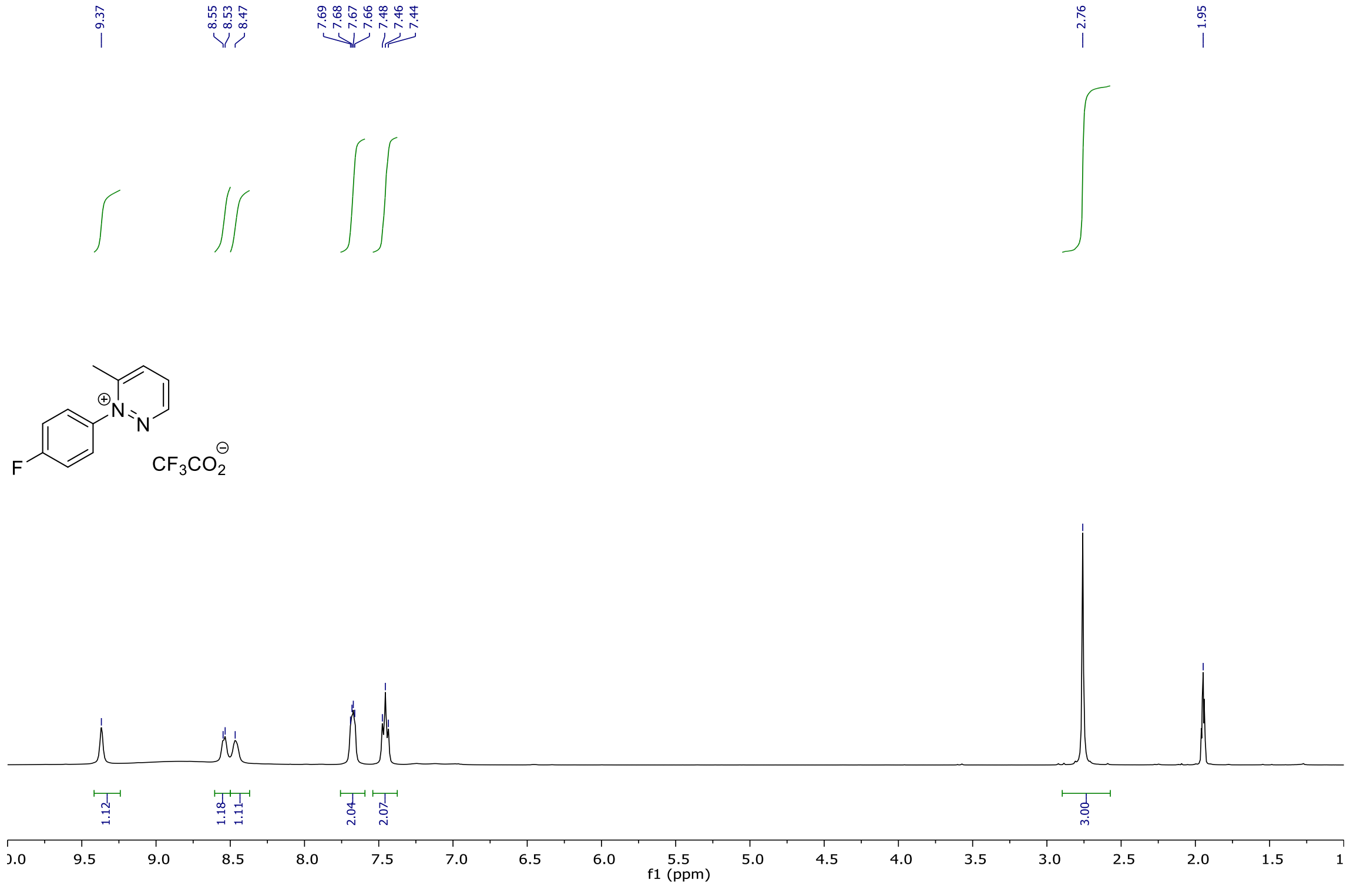

S20 
1-(4-Fluorophenyl)-6-methylpyridazin-1-ium trifluoroacetate (1e”) DEPTQ-NMR ( $\left.\mathrm{CD}_{3} \mathrm{CN}, 151 \mathrm{MHz}\right)$

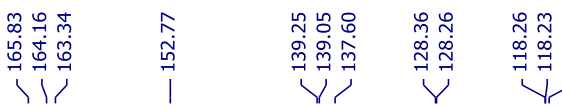
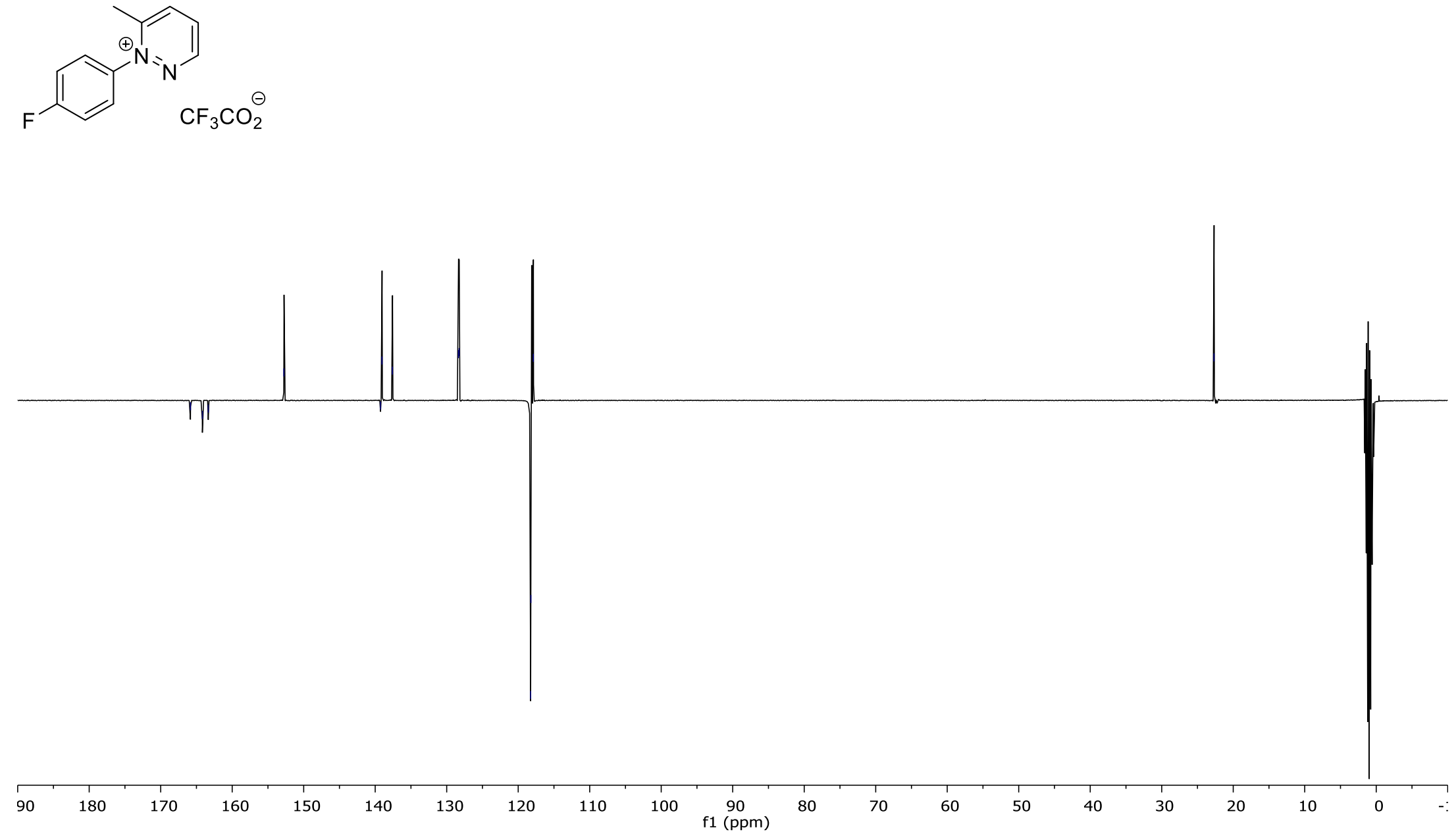
6-(Acetamidomethyl)-1-(4-fluorophenyl)pyridazin-1-ium trifluoroacetate (1f', ${ }^{1} \mathrm{H}-\mathrm{NMR}\left(\mathrm{CD}_{3} \mathrm{CN}, 400 \mathrm{MHz}\right)$

$\underbrace{\circ}$

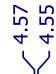
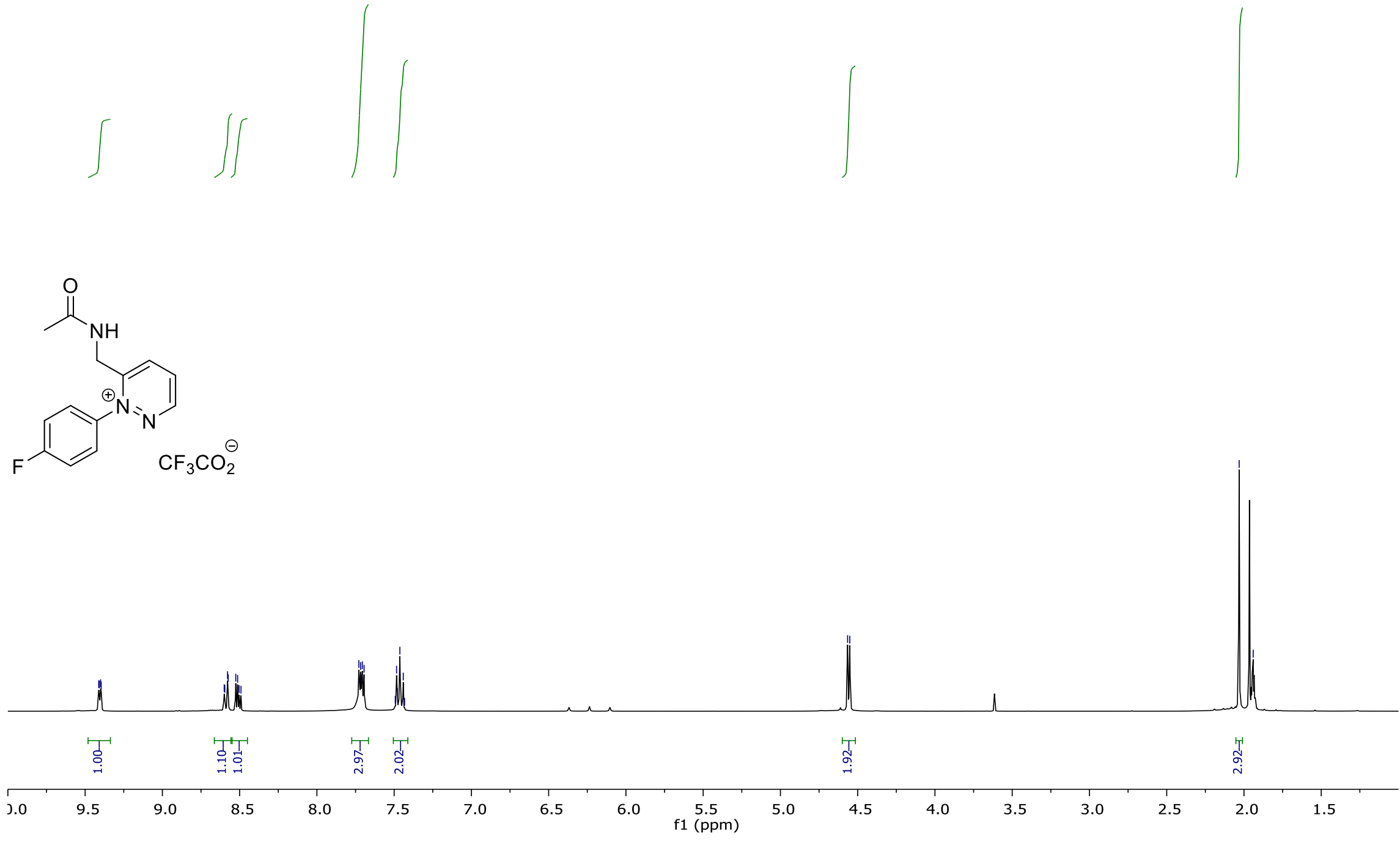

S22 
6-(Acetamidomethyl)-1-(4-fluorophenyl)pyridazin-1-ium trifluoroacetate (1f'’) DEPTQ-NMR (CD 3 CN, $101 \mathrm{MHz})$
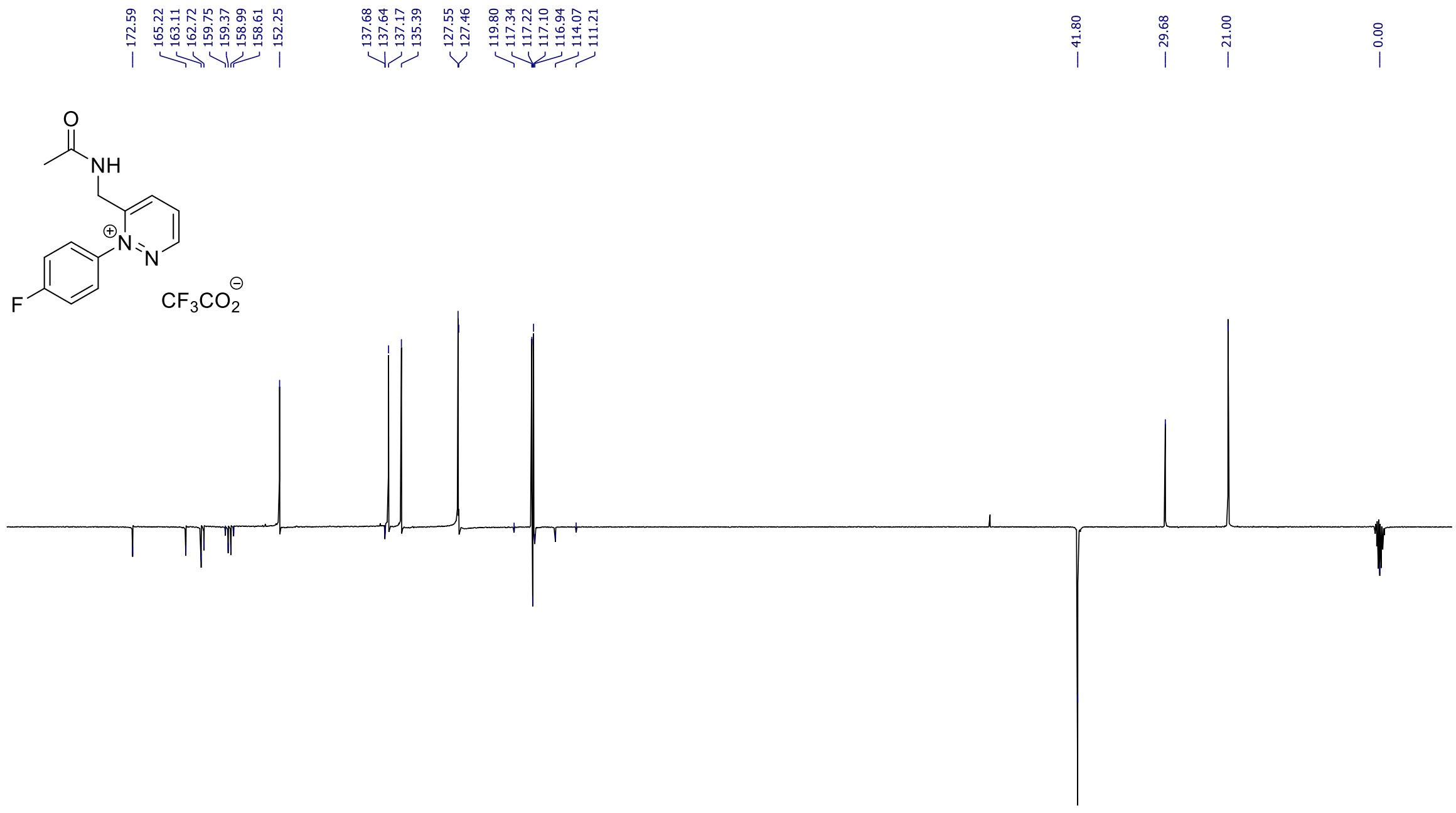

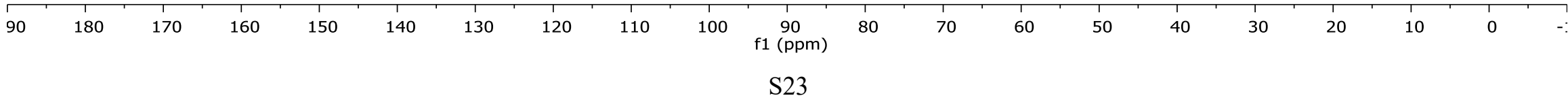


1-(4-Methoxyphenyl)-3,6-dimethylpyridazin-1-ium trifluoroacetate (1g’’) ${ }^{1} \mathrm{H}-\mathrm{NMR}\left(\mathrm{CD}_{3} \mathrm{CN}, 400 \mathrm{MHz}\right)$
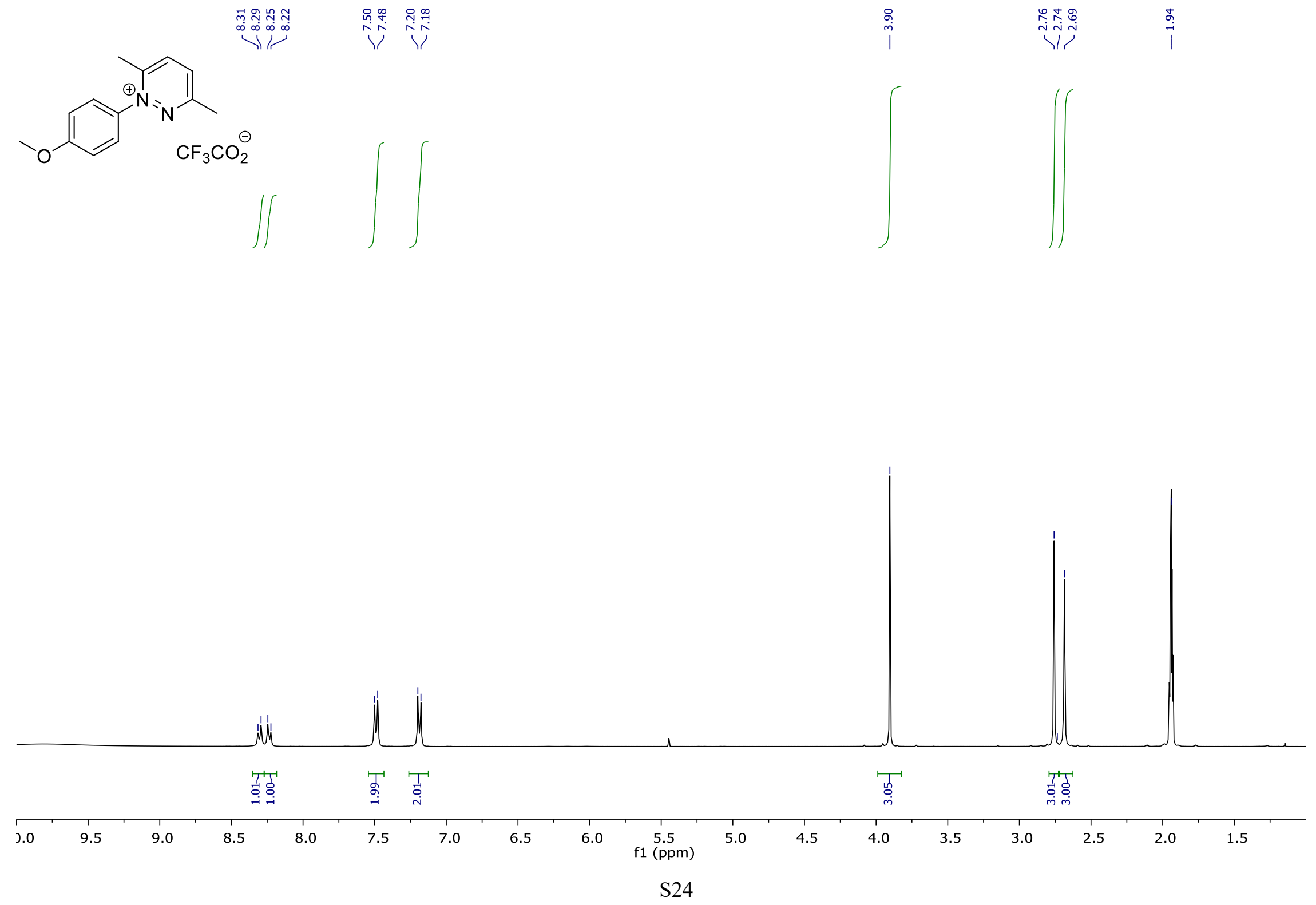
1-(4-Methoxyphenyl)-3,6-dimethylpyridazin-1-ium trifluoroacetate (1g’’) DEPTQ-NMR (CD 3 CN, $151 \mathrm{MHz})$

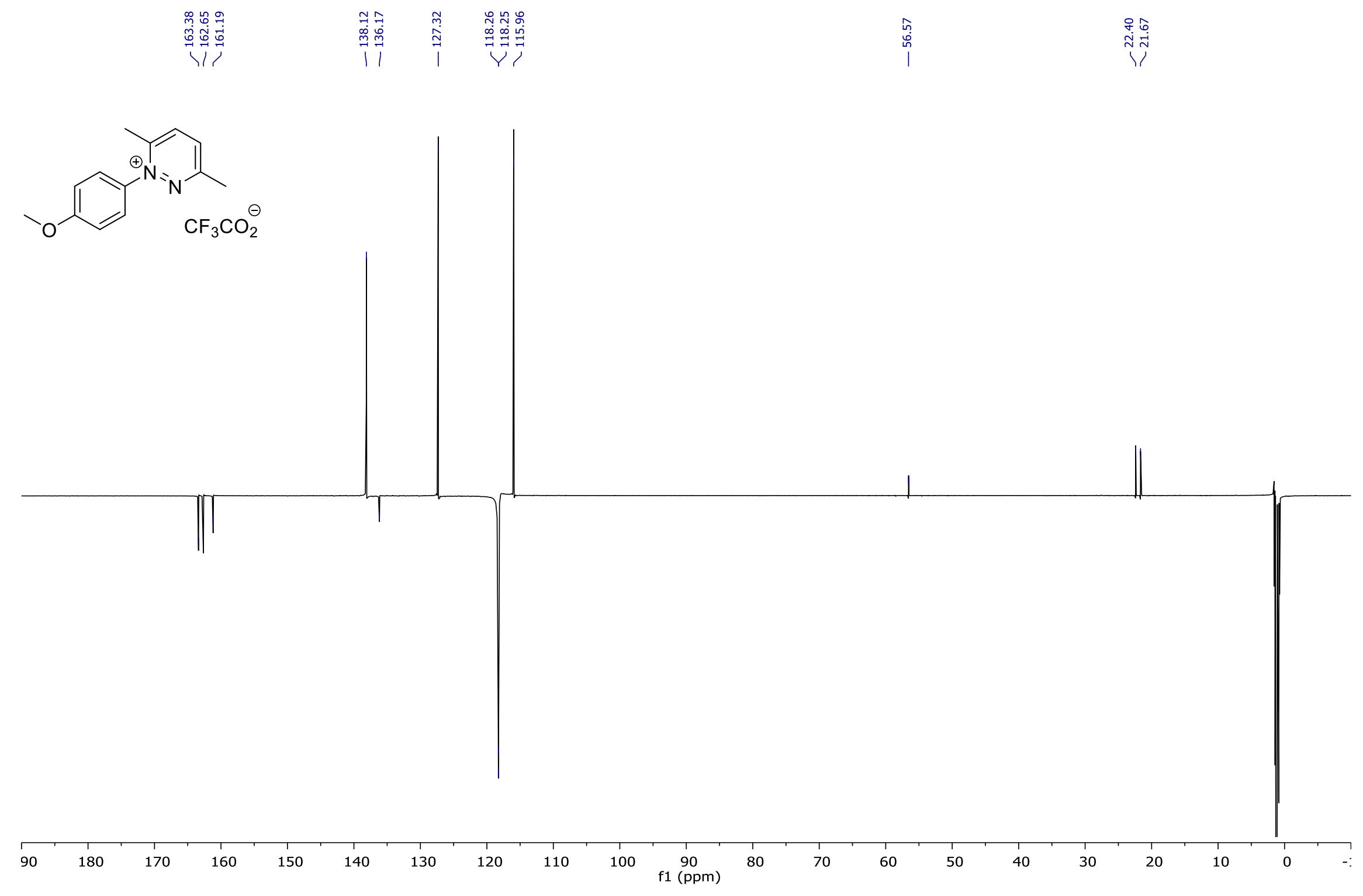


1-(4-Methoxyphenyl)-6-methylpyridazin-1-ium trifluoroacetate (1h") 1H-NMR (CD3CN, $400 \mathrm{MHz})$
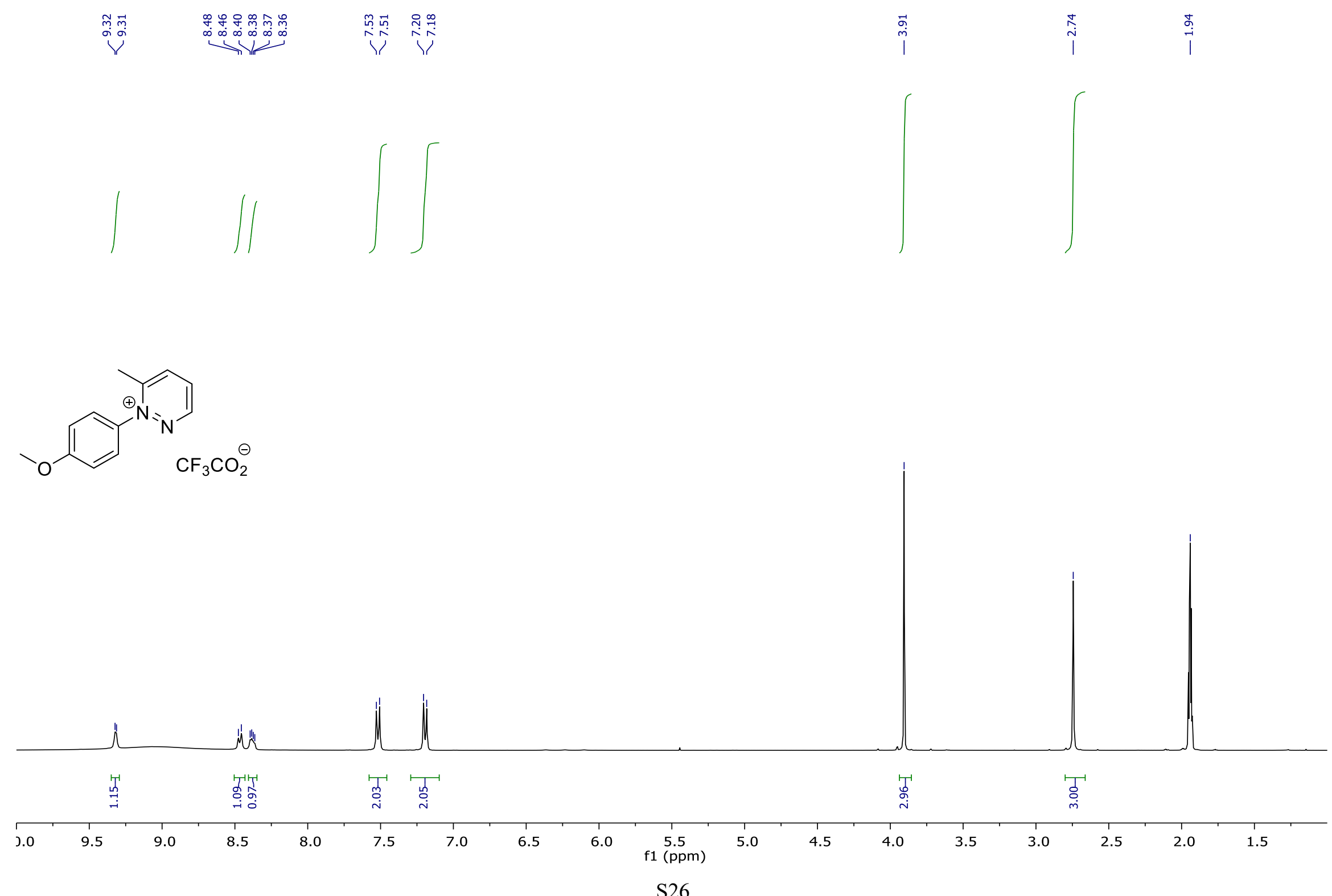
1-(4-Methoxyphenyl)-6-methylpyridazin-1-ium trifluoroacetate (1h”) DEPTQ-NMR (CD 3 CN, $151 \mathrm{MHz})$

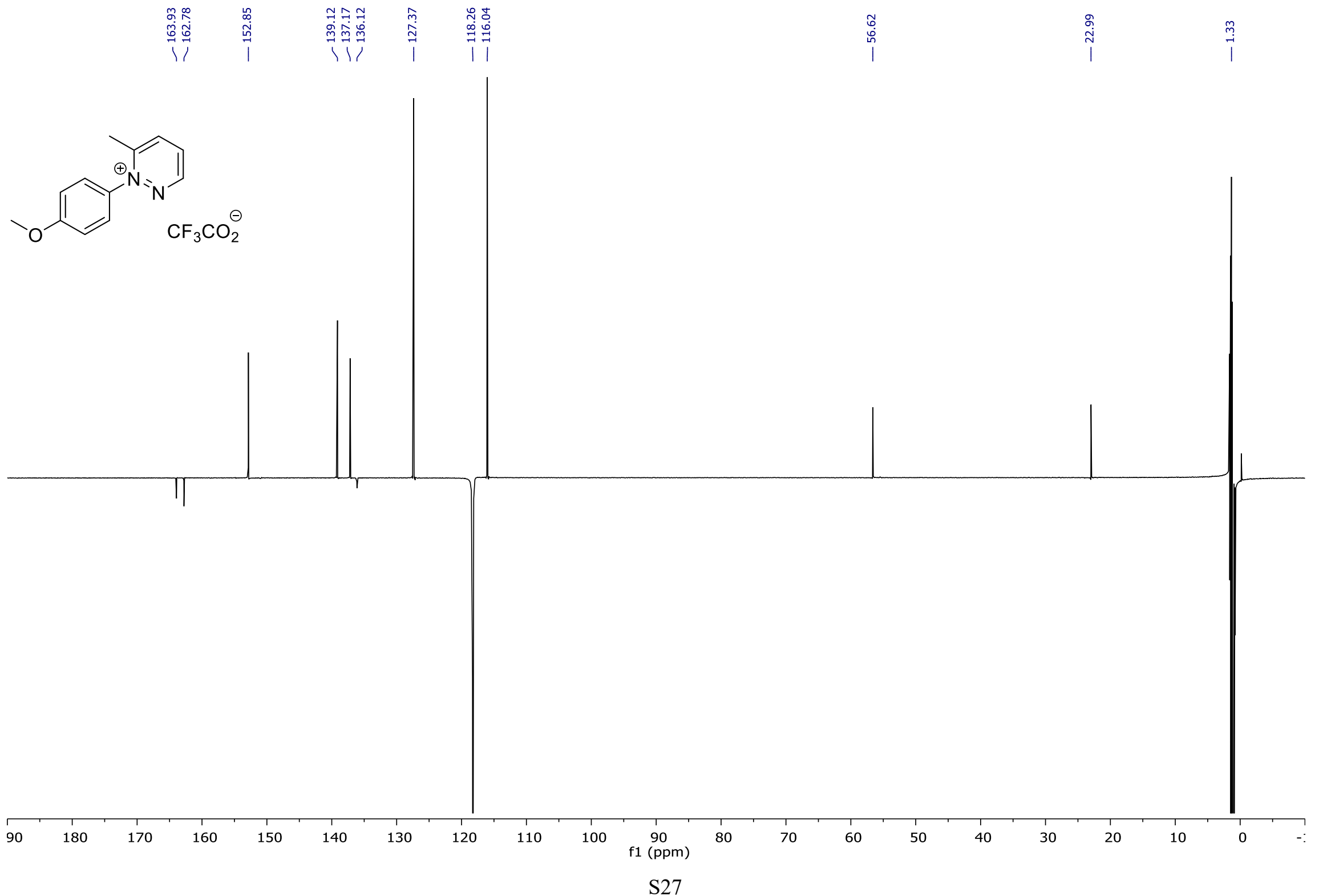


6-(Acetamidomethyl)-1-(4-methoxyphenyl)pyridazin-1-ium trifluoroacetate (1i'”) ${ }^{1} \mathrm{H}-\mathrm{NMR}\left(\mathrm{CD}_{3} \mathrm{CN}, 400 \mathrm{MHz}\right)$

$\underbrace{\infty}$

$\iiint$

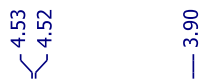

$\stackrel{\circ}{\stackrel{\circ}{g}+j}$

(1)

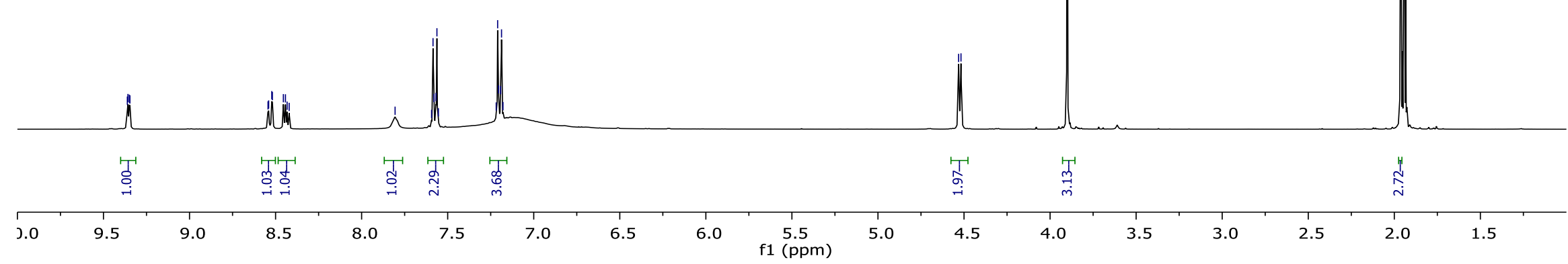


6-(Acetamidomethyl)-1-(4-methoxyphenyl)pyridazin-1-ium trifluoroacetate (1i’”) DEPTQ-NMR (CD $\left.{ }_{3} \mathrm{CN}, 101 \mathrm{MHz}\right)$

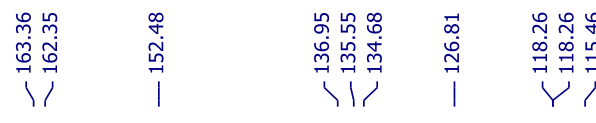
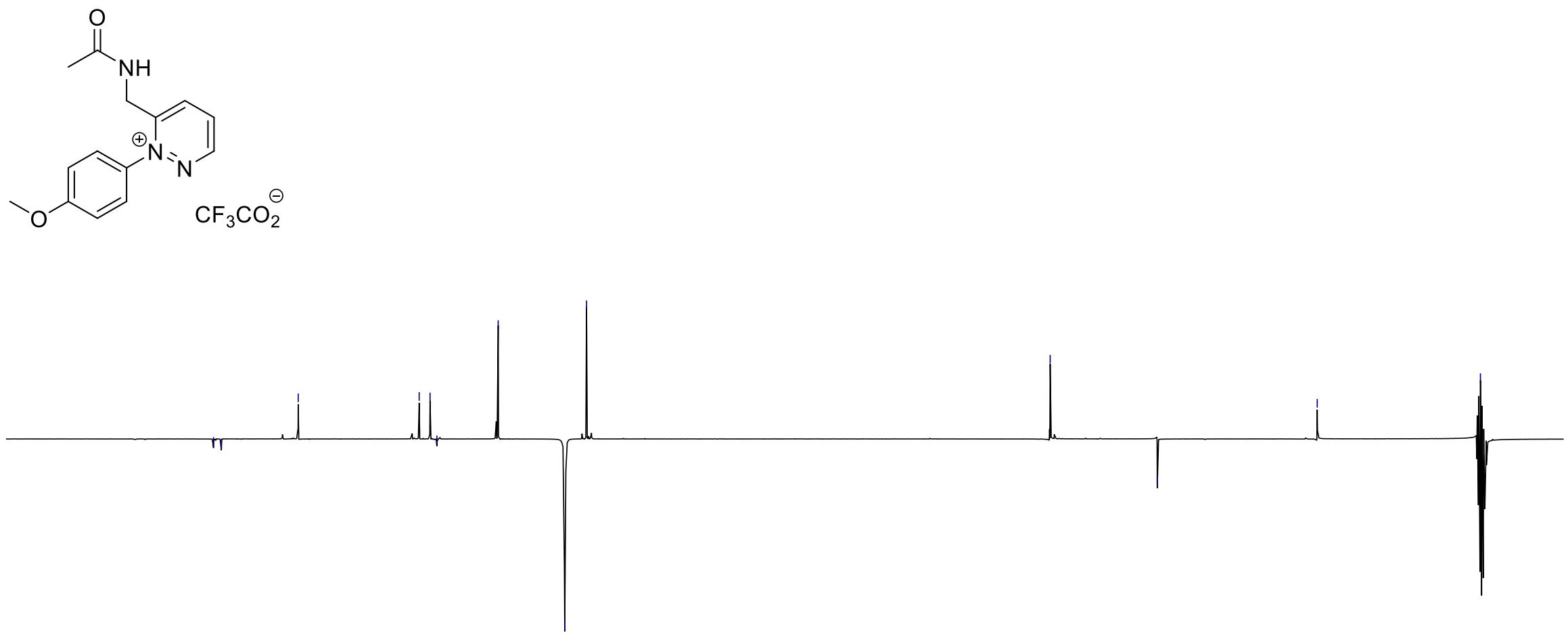
6-(Acetamidomethyl)-1-(4-methoxyphenyl)pyridazin-1-ium trifluoroacetate (1i'”) selective NOE (CD 3 CN, $600 \mathrm{MHz}, 4.55 \mathrm{ppm})$

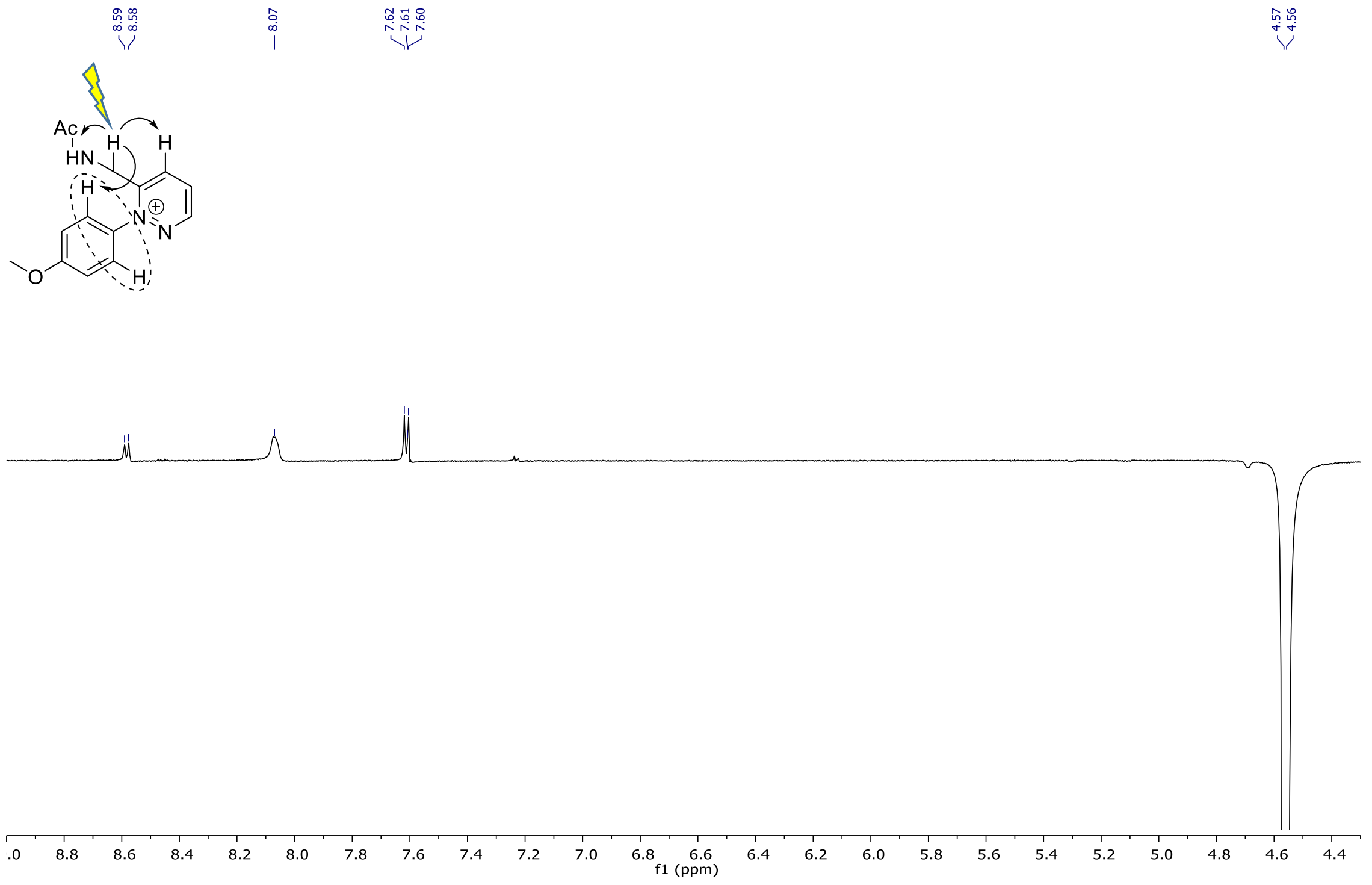


1-(4-Cyanophenyl)-3,6-dimethylpyridazin-1-ium trifluoroacetate (1j”) ${ }^{1} \mathrm{H}-\mathrm{NMR}\left(\mathrm{CD}_{3} \mathrm{CN}, 400 \mathrm{MHz}\right)$
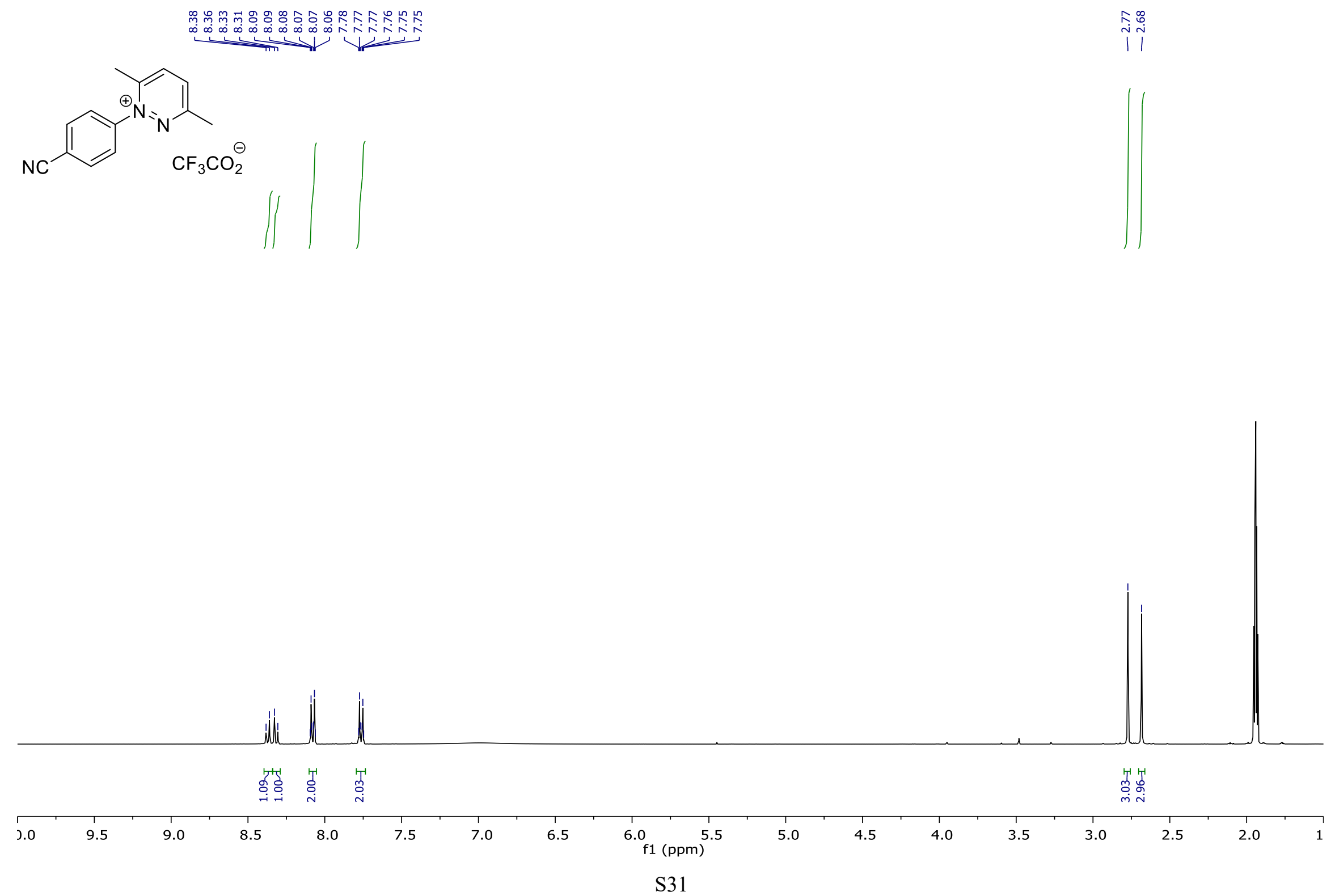
1-(4-Cyanophenyl)-3,6-dimethylpyridazin-1-ium trifluoroacetate (1j”) DEPTQ-NMR (CD 3 CN, $151 \mathrm{MHz})$

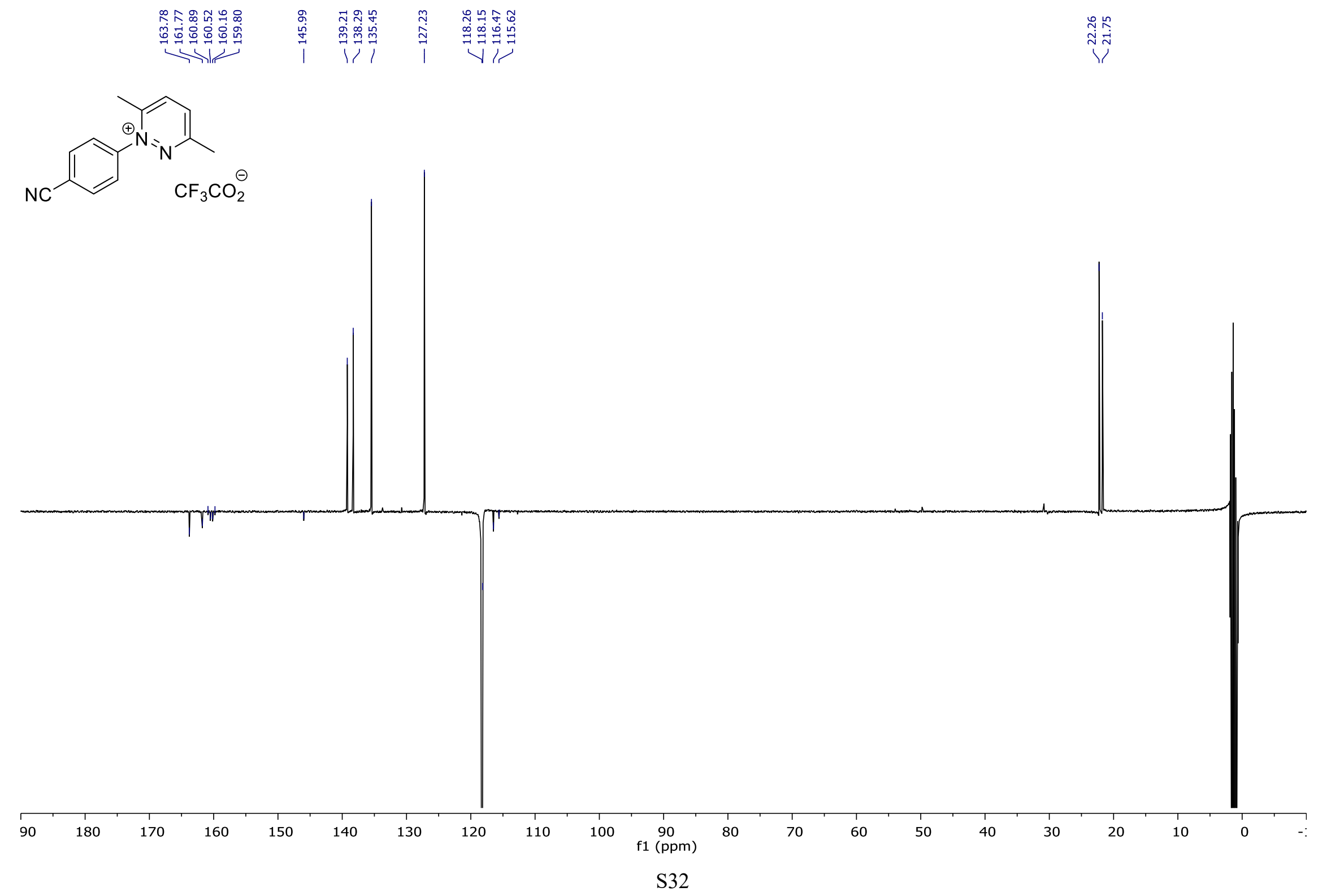


1-(4-Cyanophenyl)-6-methylpyridazin-1-ium trifluoroacetate (1k") ${ }^{1} \mathrm{H}-\mathrm{NMR}\left(\mathrm{CD}_{3} \mathrm{CN}, 400 \mathrm{MHz}\right)$

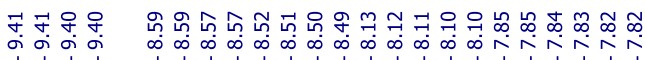

(1)

$\int \quad \iint 5$

$\overbrace{\mathrm{CF}_{3} \mathrm{CO}_{2}^{\ominus}}^{\Theta}$

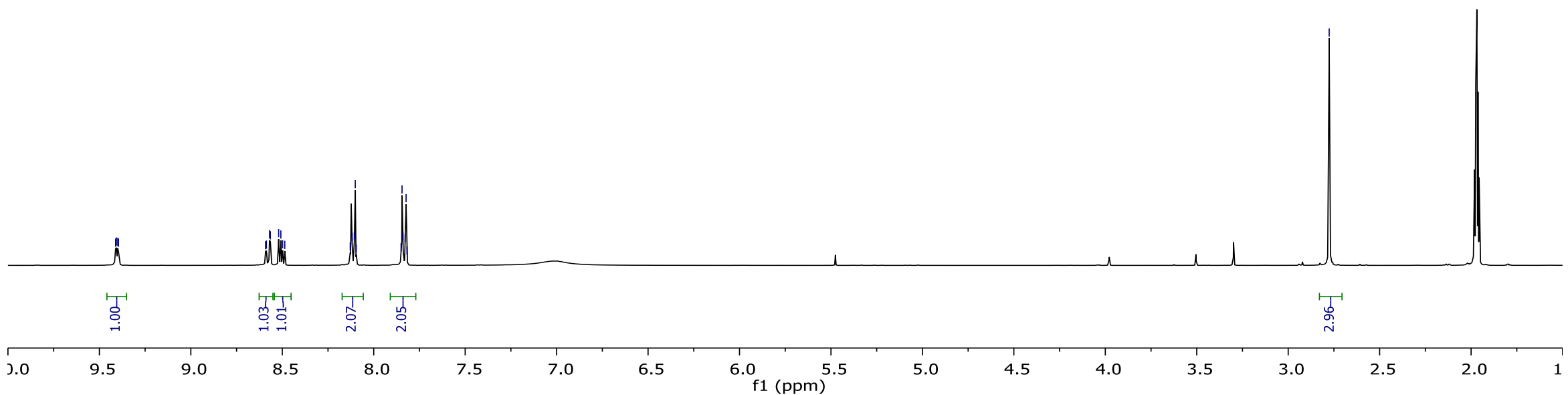


1-(4-Cyanophenyl)-6-methylpyridazin-1-ium trifluoroacetate (1k”) DEPTQ-NMR $\left(\mathrm{CD}_{3} \mathrm{CN}, 151 \mathrm{MHz}\right)$

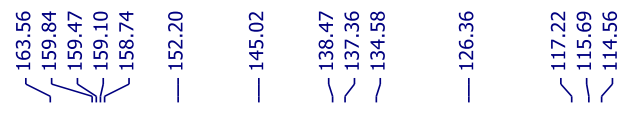
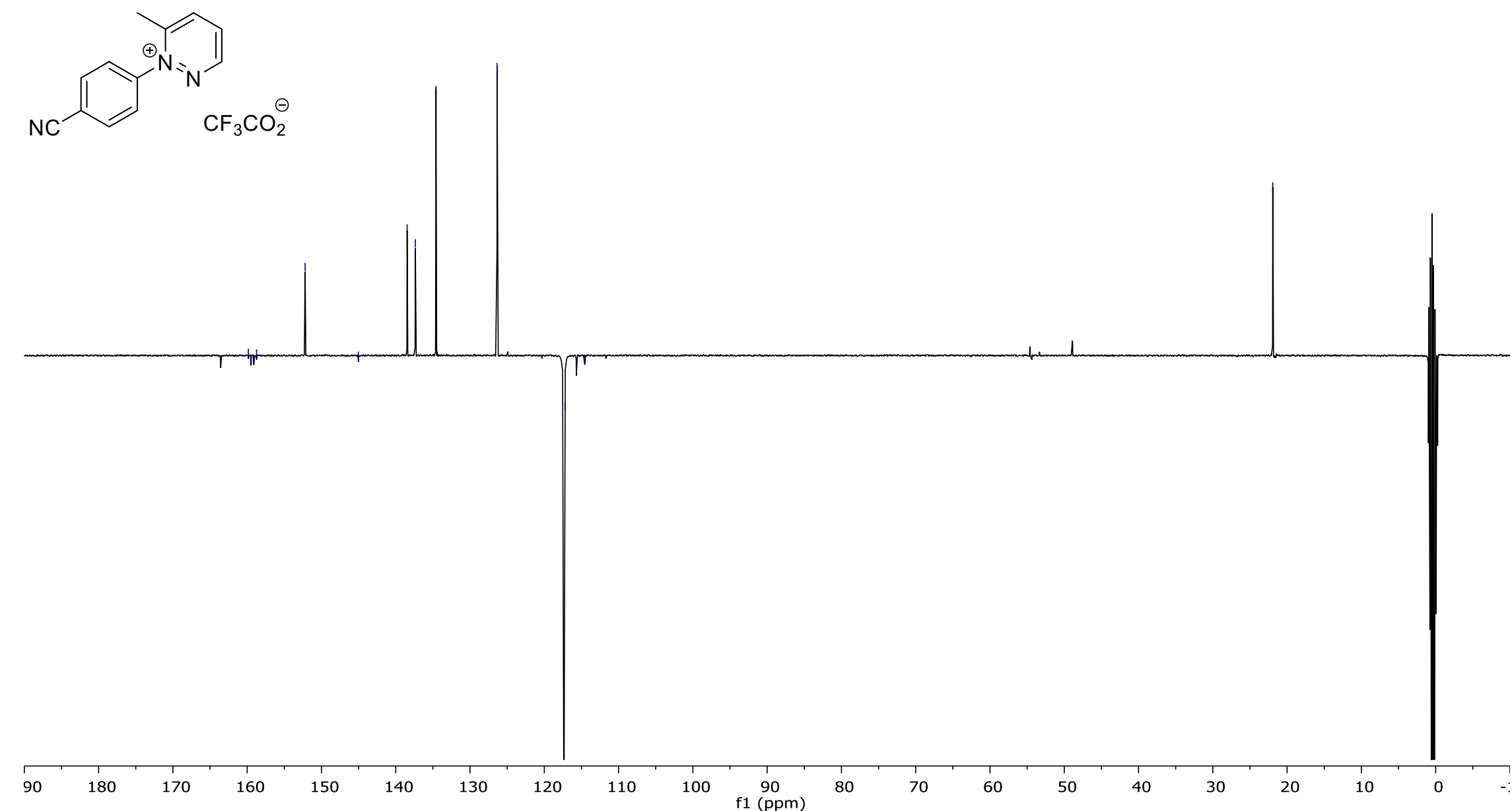
6-(Acetamidomethyl)-1-(4-cyanophenyl)pyridazin-1-ium trifluoroacetate (11'’) ${ }^{1} \mathrm{H}-\mathrm{NMR}\left(\mathrm{CD}_{3} \mathrm{CN}, 600 \mathrm{MHz}\right)$
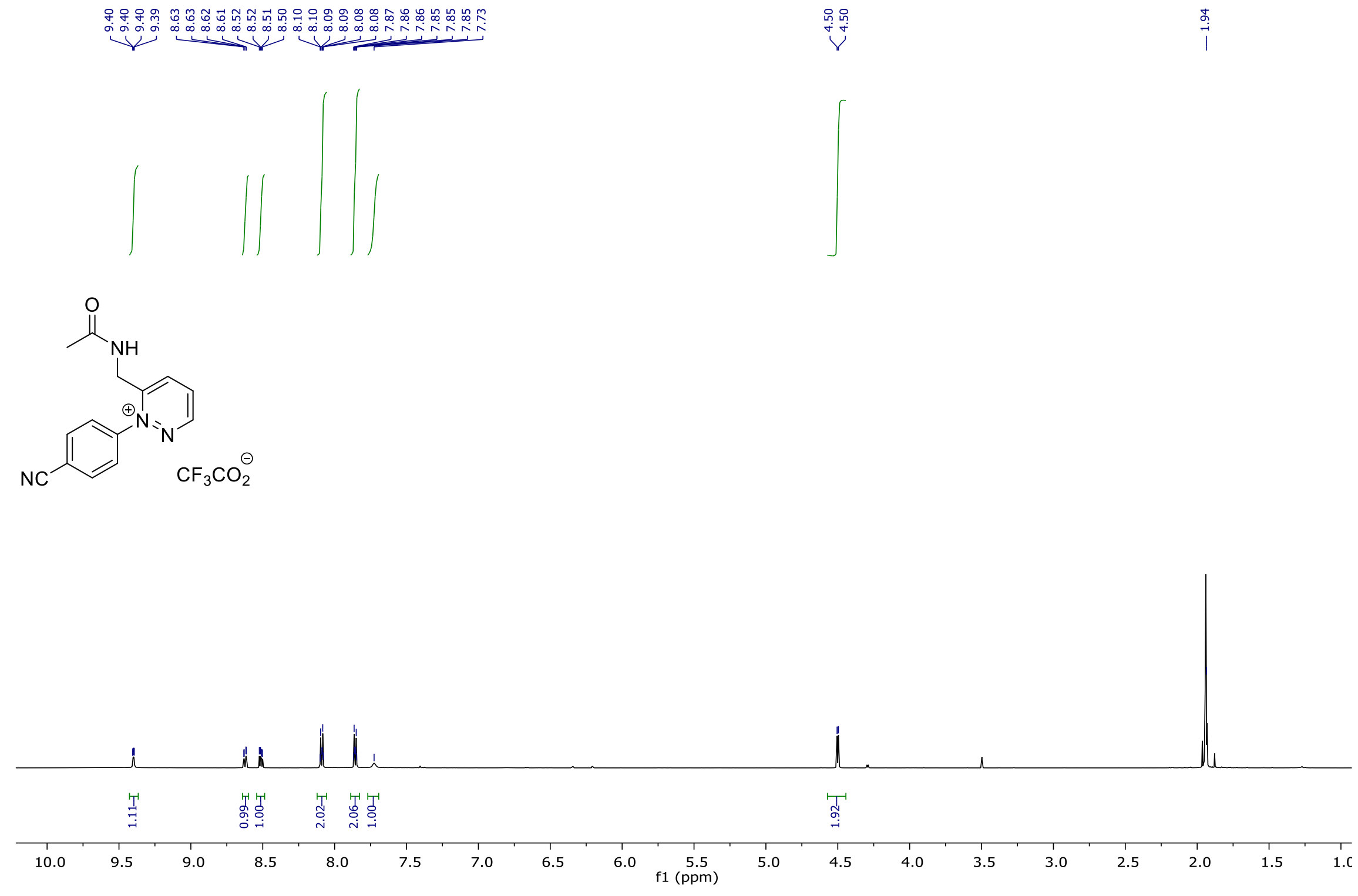
6-(Acetamidomethyl)-1-(4-cyanophenyl)pyridazin-1-ium trifluoroacetate (11’') DEPTQ-NMR (CD $\left.{ }_{3} \mathrm{CN}, 151 \mathrm{MHz}\right)$

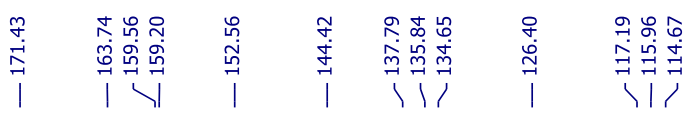

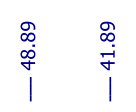

$\stackrel{\substack{n \\ i}}{1}$<smiles></smiles>

90

180

170

160

150

140

130

120

110

100

90

$80 \quad 70$

60

30

20

10

S36 
6-(Acetamidomethyl)-1-(4-cyanophenyl)pyridazin-1-ium trifluoroacetate (11’') selectiv NOE (CD 3 CN, $600 \mathrm{MHz}, 4.55 \mathrm{ppm})$

$\underbrace{1}$
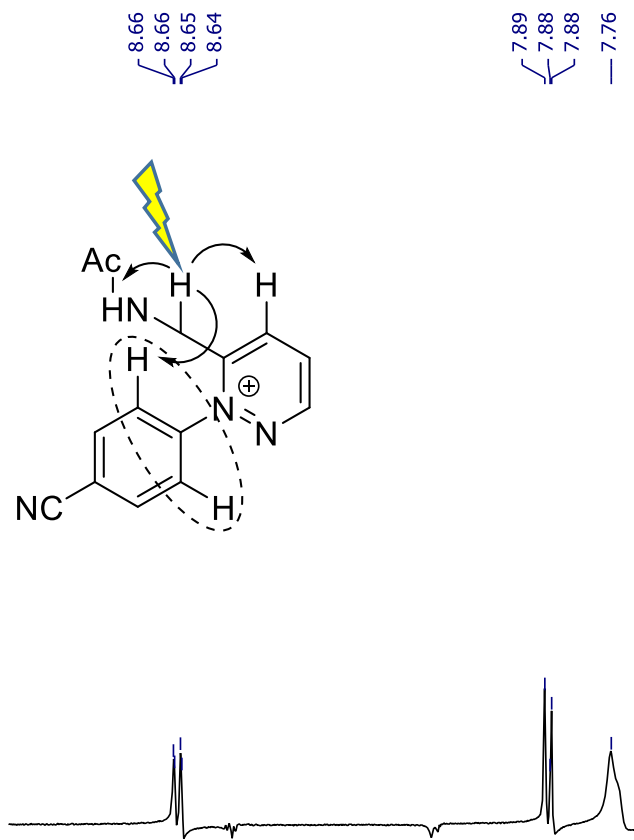
3,6-dimethyl-1-phenylpyridazin-1-ium trifluoroacetate (1 m'") ${ }^{1} \mathrm{H}-\mathrm{NMR}\left(\mathrm{CD}_{3} \mathrm{CN}, 400 \mathrm{MHz}\right)$

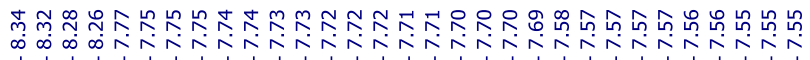

U1/

$\overbrace{\mathrm{CF}_{3} \mathrm{CO}_{2}^{\ominus}}^{\ominus}$
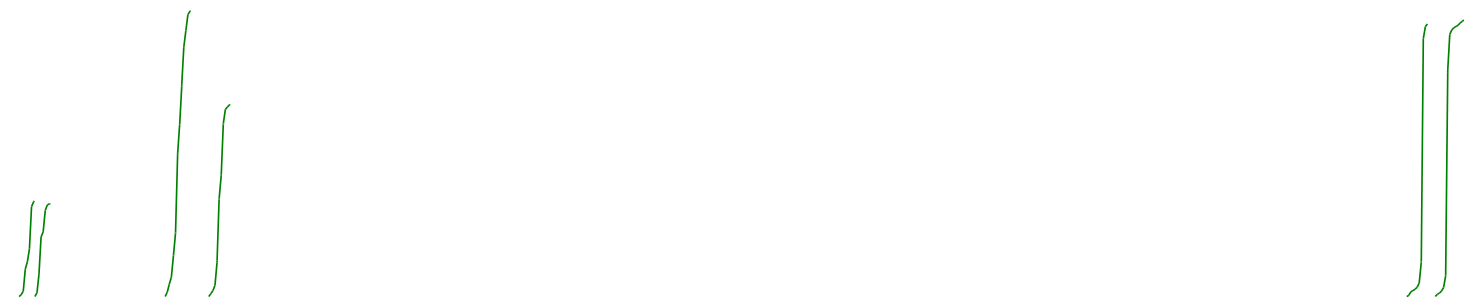

in

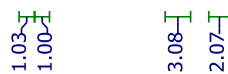

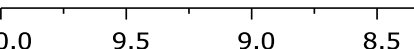

$8.0 \quad 7.5$

7.0

6.5

6.0

5.5
(ppm) 
3,6-dimethyl-1-phenylpyridazin-1-ium trifluoroacetate (1 m’') DEPTQ-NMR $\left(\mathrm{CD}_{3} \mathrm{CN}, 101 \mathrm{MHz}\right)$

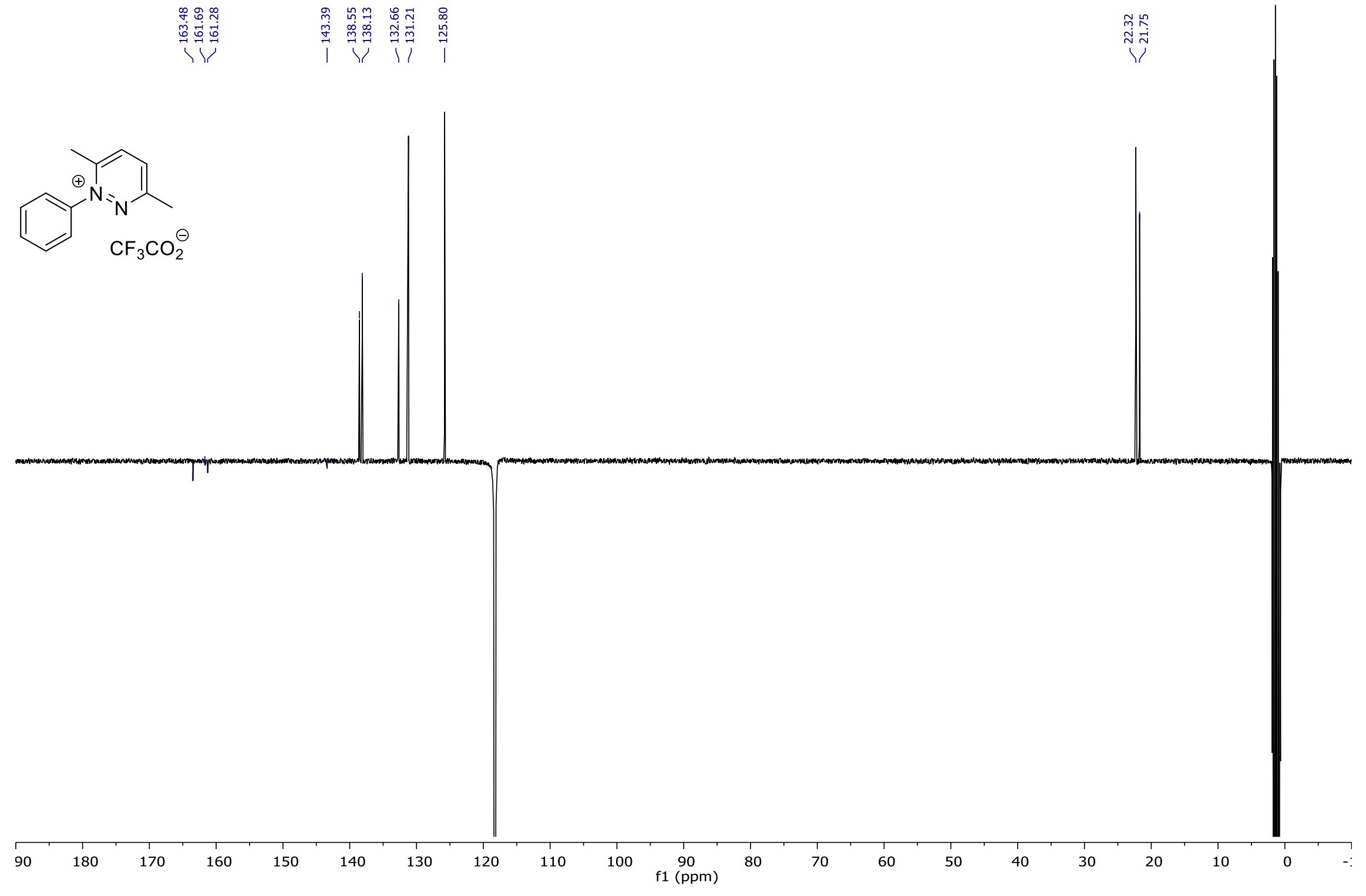


6-methyl-1-phenylpyridazin-1-ium trifluoroacetate (1n'”) ${ }^{1} \mathrm{H}-\mathrm{NMR}\left(\mathrm{CD}_{3} \mathrm{CN}, 400 \mathrm{MHz}\right)$

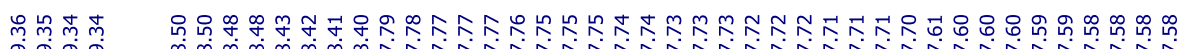

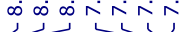

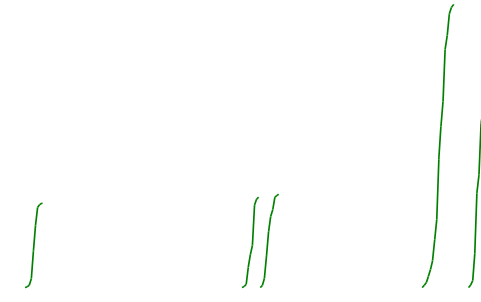

$\overbrace{\mathrm{CF}_{3} \mathrm{CO}_{2}}^{\ominus}$

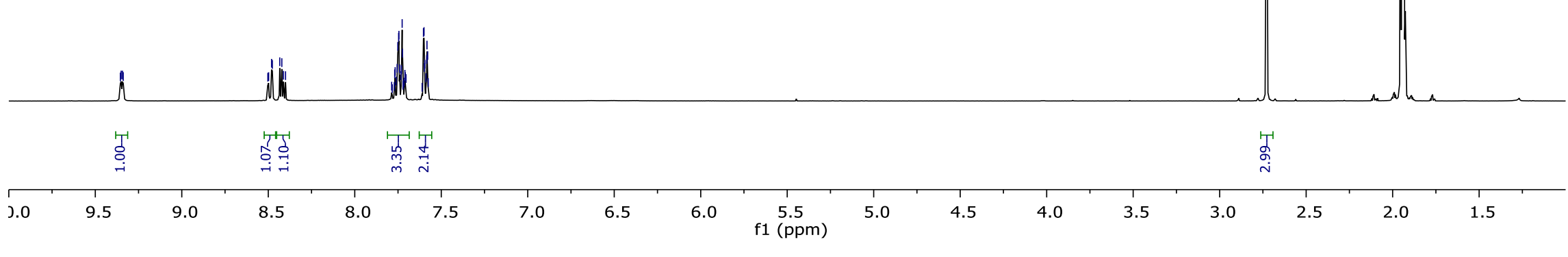


6-methyl-1-phenylpyridazin-1-ium trifluoroacetate (1n”) DEPTQ-NMR $\left(\mathrm{CD}_{3} \mathrm{CN}, 101 \mathrm{MHz}\right)$

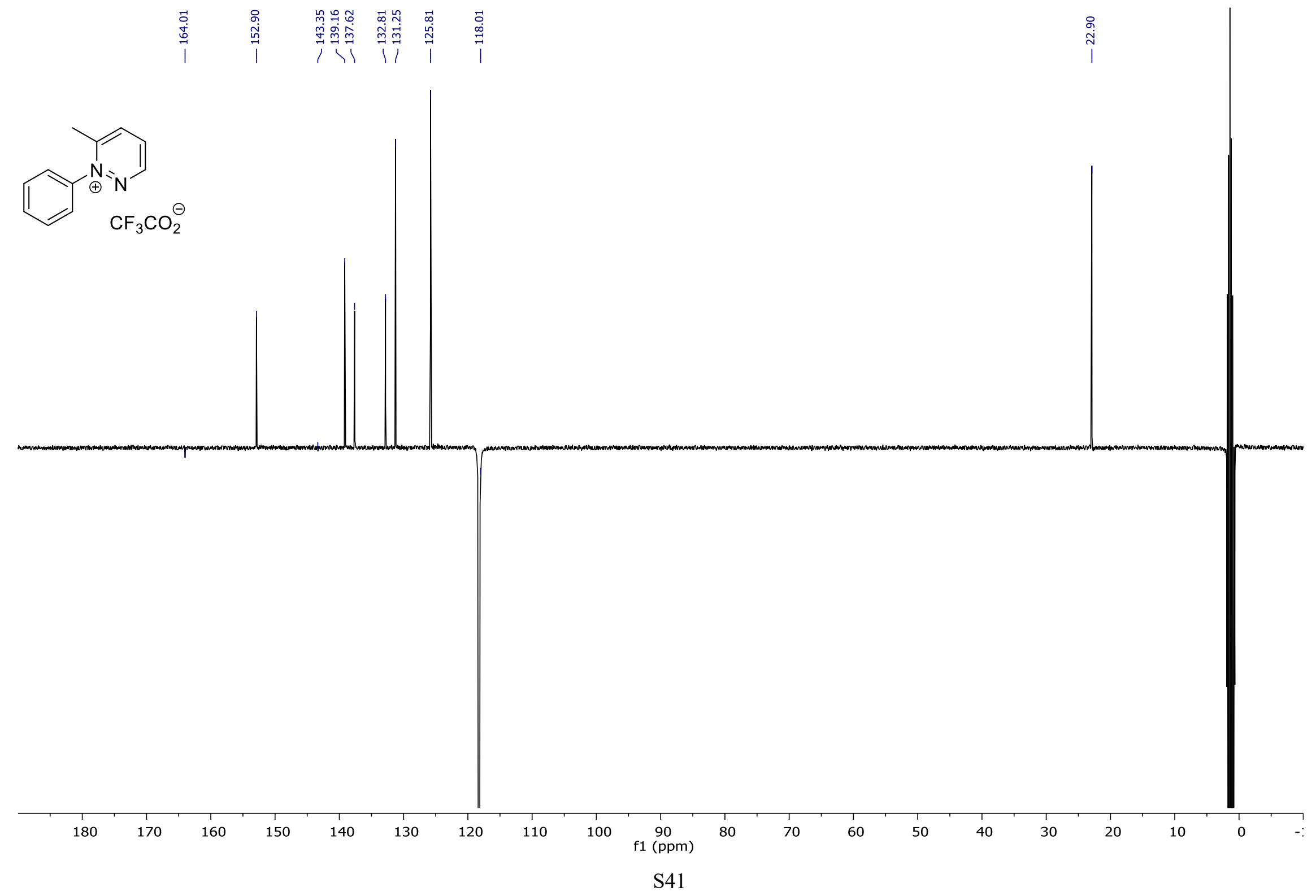


6-(acetamidomethyl)-1-phenylpyridazin-1-ium trifluoroacetate (10") ${ }^{1} \mathrm{H}-\mathrm{NMR}\left(\mathrm{CD}_{3} \mathrm{CN}, 400 \mathrm{MHz}\right)$

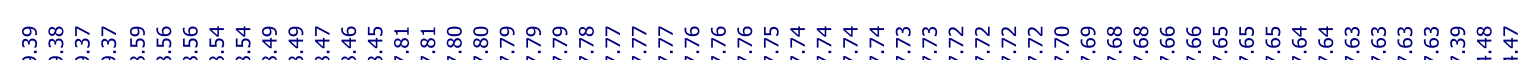
$V_{0} \underbrace{\infty}$
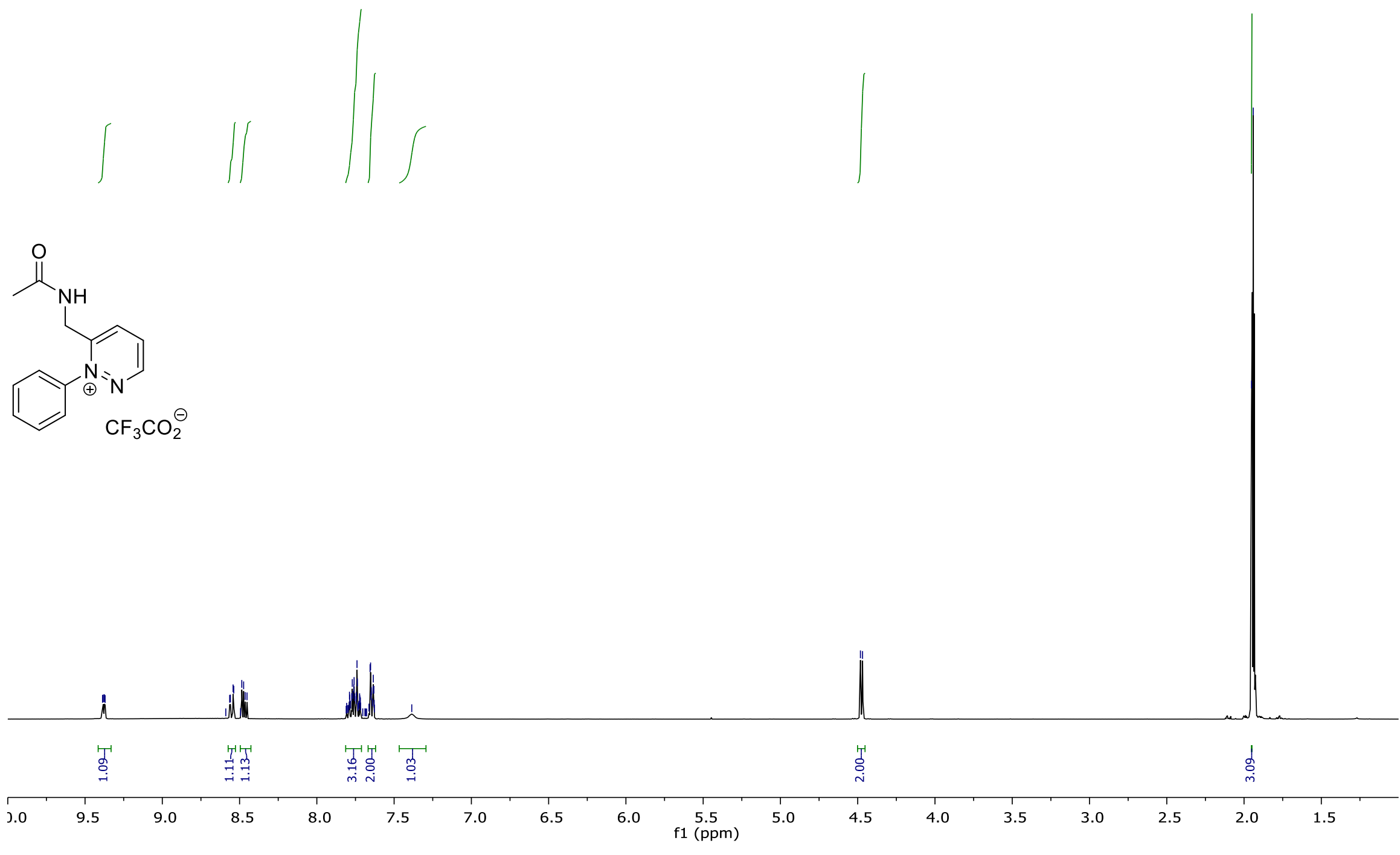
6-(acetamidomethyl)-1-phenylpyridazin-1-ium trifluoroacetate (1o'”) DEPTQ-NMR $\left(\mathrm{CD}_{3} \mathrm{CN}, 101 \mathrm{MHz}\right)$

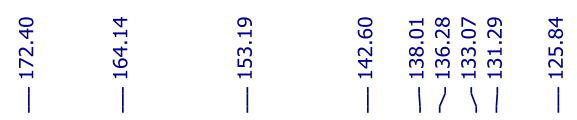
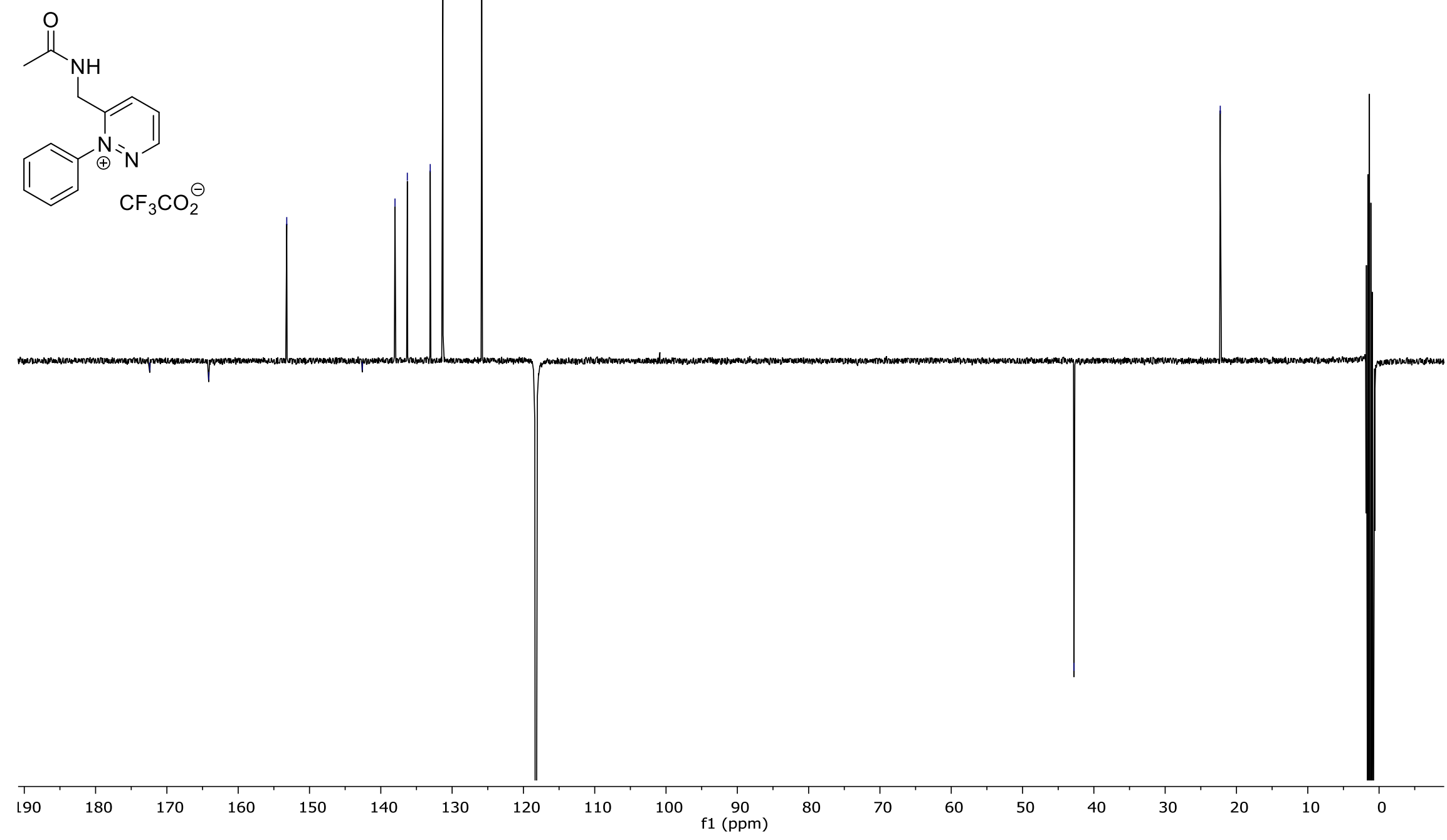


\section{Spectra of pyridazinium methanesulfonates (1a'-l')}

1-(4-Chlorophenyl)-3,6-dimethylpyridazin-1-ium methanesulfonate (1a') ${ }^{1} \mathrm{H}-\mathrm{NMR}$ (CD3CN, $\left.400 \mathrm{MHz}\right)$

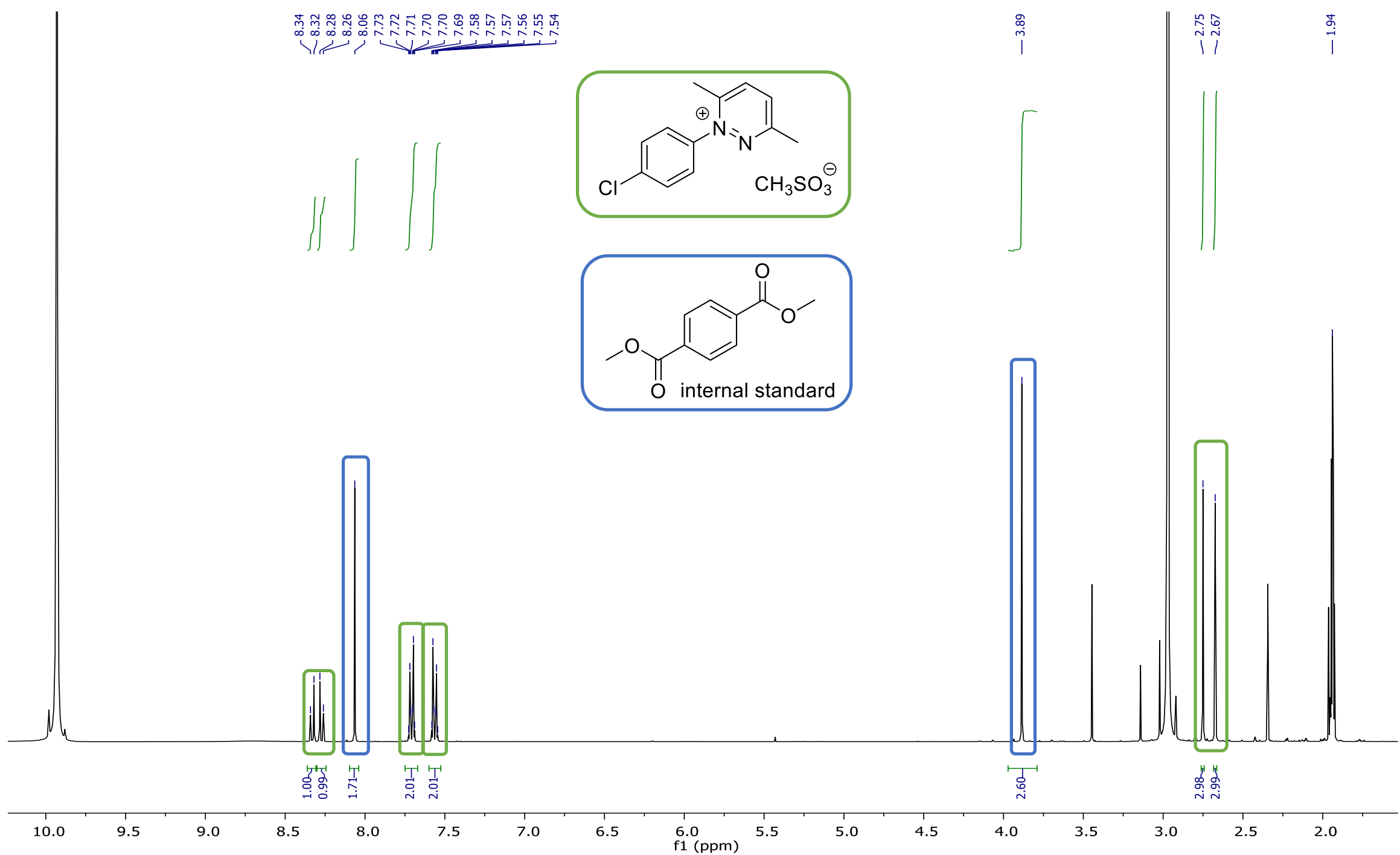


1-(4-Chlorophenyl)-6-methylpyridazin-1-ium methanesulfonate (1b') ${ }^{1} \mathrm{H}-\mathrm{NMR}\left(\mathrm{CD}_{\mathbf{3}} \mathrm{CN}, 400 \mathrm{MHz}\right)$

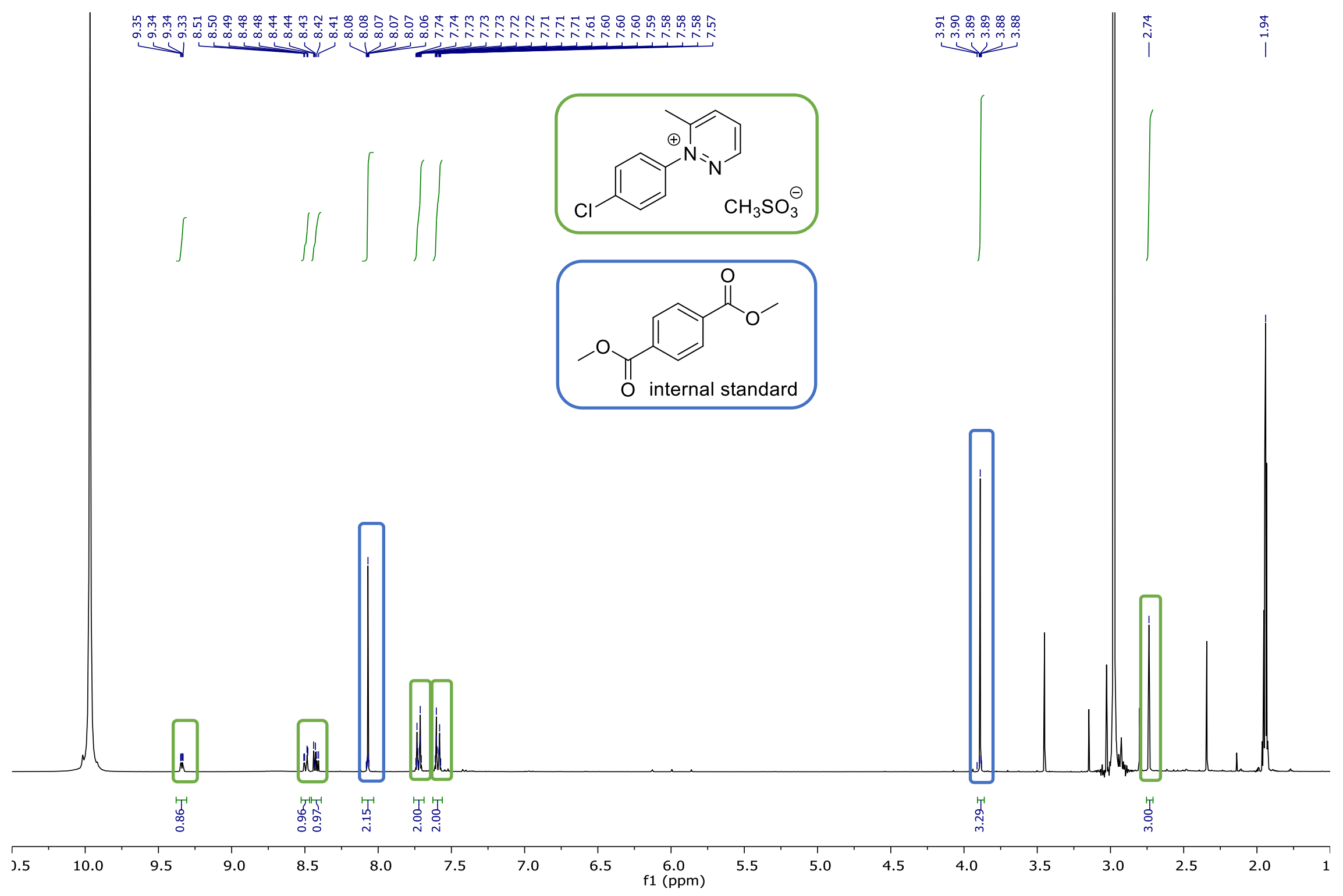


6-(Acetamidomethyl)-1-(4-chlorophenyl)pyridazin-1-ium methanesulfonate (1c') ${ }^{1} \mathrm{H}-\mathrm{NMR}\left(\mathrm{CD}_{3} \mathrm{CN}, 400 \mathrm{MHz}\right)$

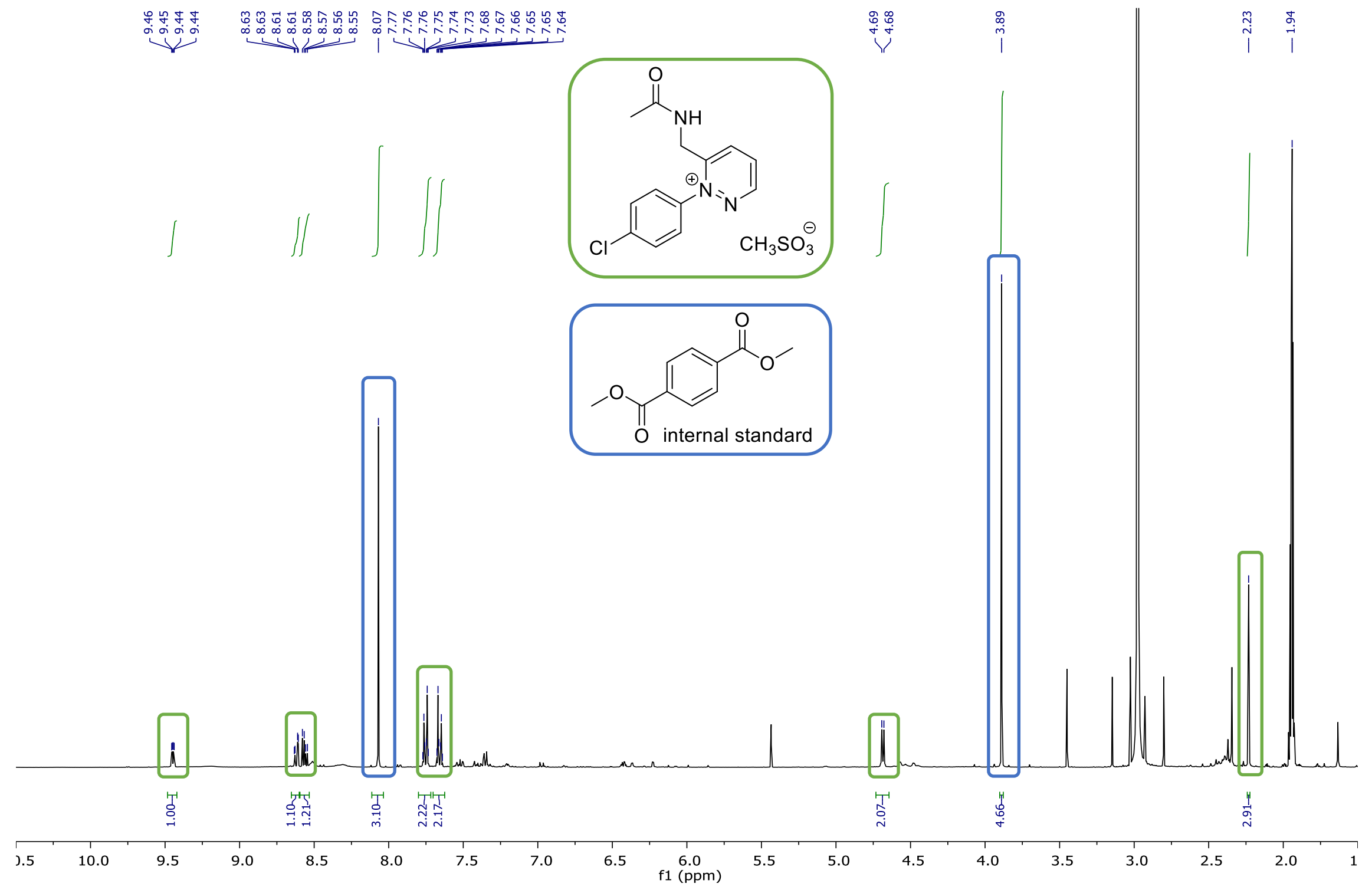


1-(4-Fluorophenyl)-3,6-dimethylpyridazin-1-ium methanesulfonate (1d') ${ }^{1} \mathrm{H}-\mathrm{NMR}\left(\mathrm{CD}_{3} \mathrm{CN}, 400 \mathrm{MHz}\right)$

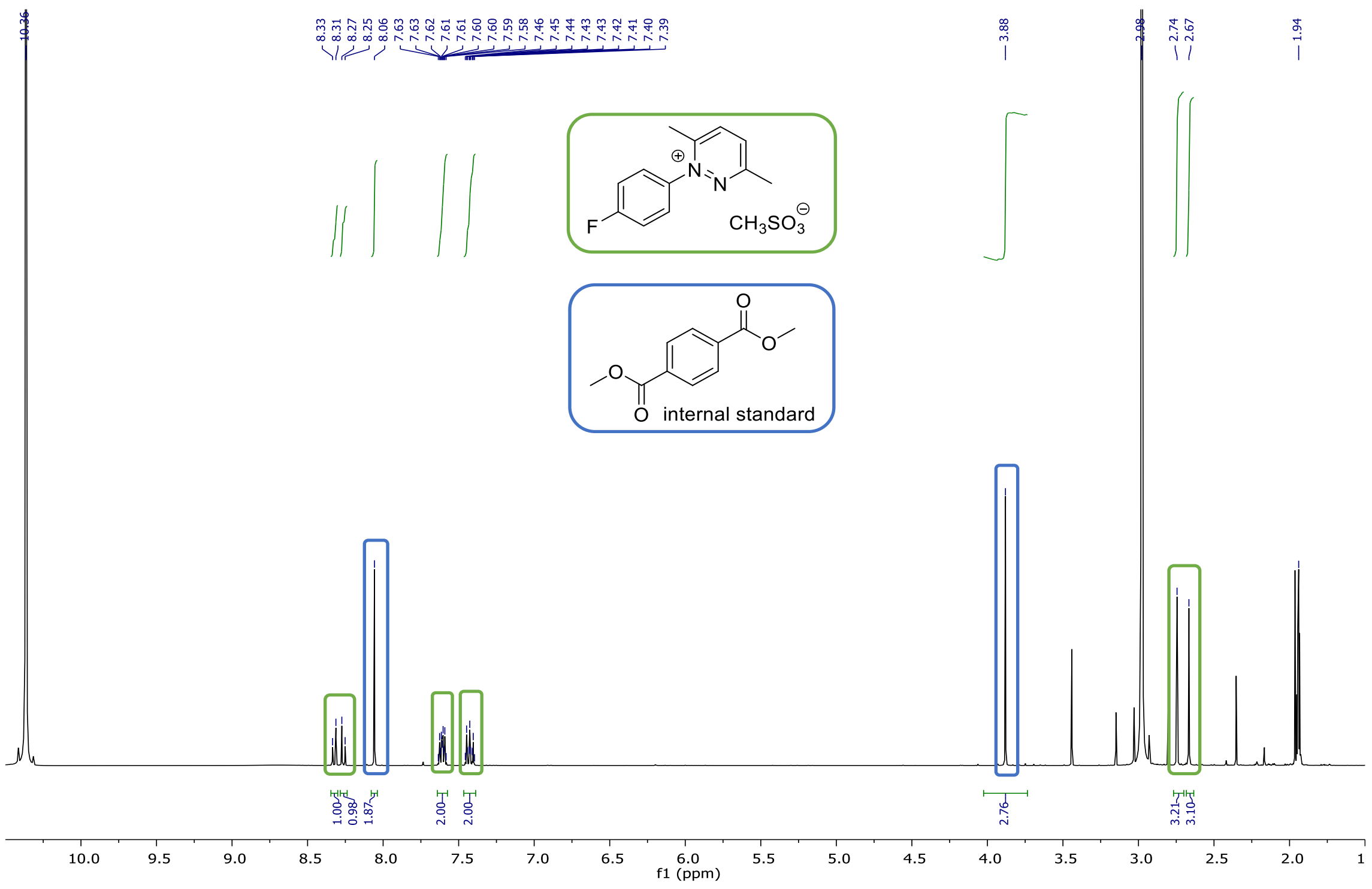


1-(4-Fluorophenyl)-6-methylpyridazin-1-ium methanesulfonate (1e') ${ }^{1} \mathrm{H}-\mathrm{NMR}\left(\mathrm{CD}_{3} \mathrm{CN}, 400 \mathrm{MHz}\right)$

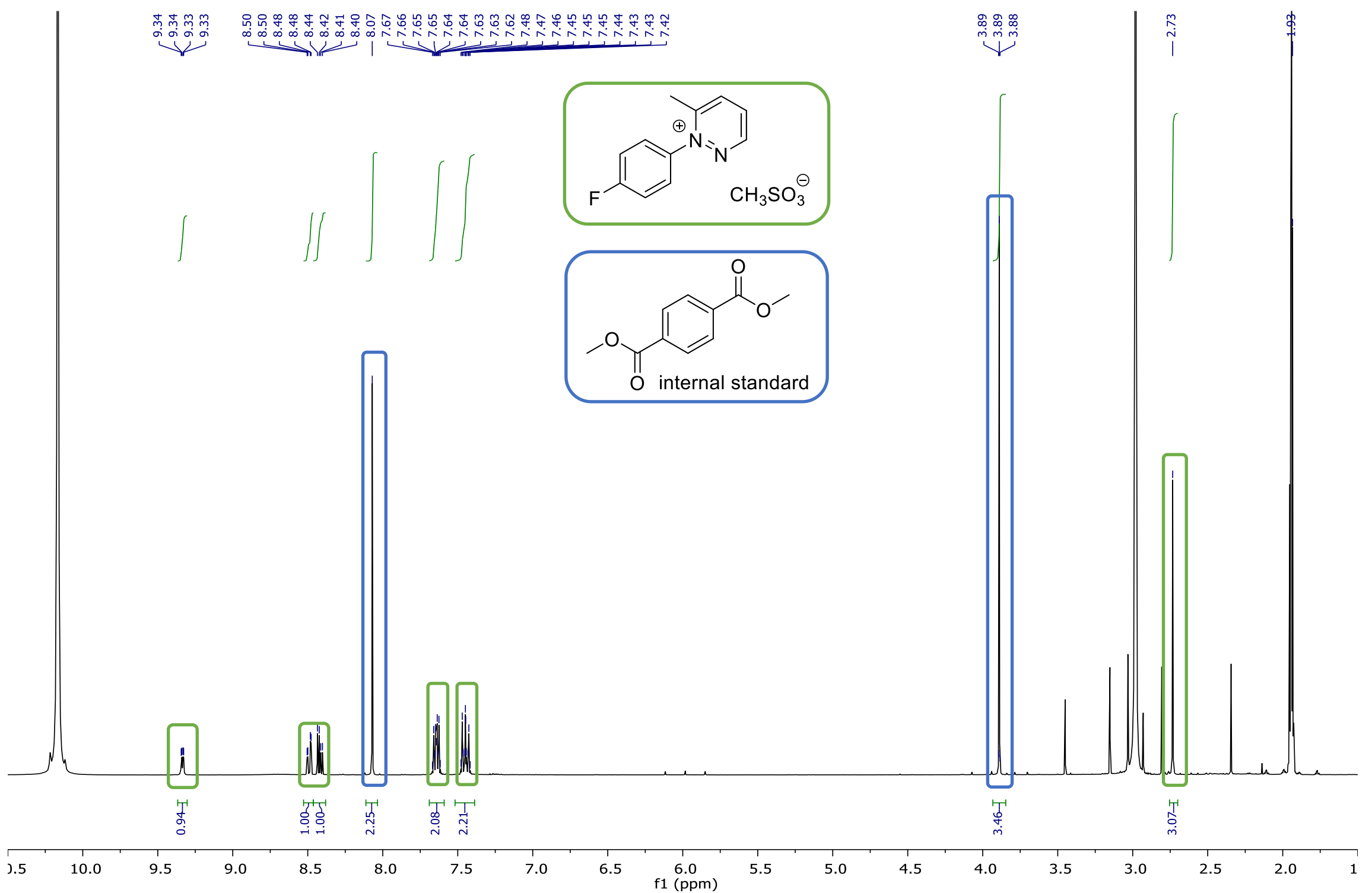


6-(Acetamidomethyl)-1-(4-fluorophenyl)pyridazin-1-ium methanesulfonate (1f') ${ }^{1} \mathrm{H}-\mathrm{NMR}$ (CD $\left.\mathrm{CN}, 400 \mathrm{MHz}\right)$

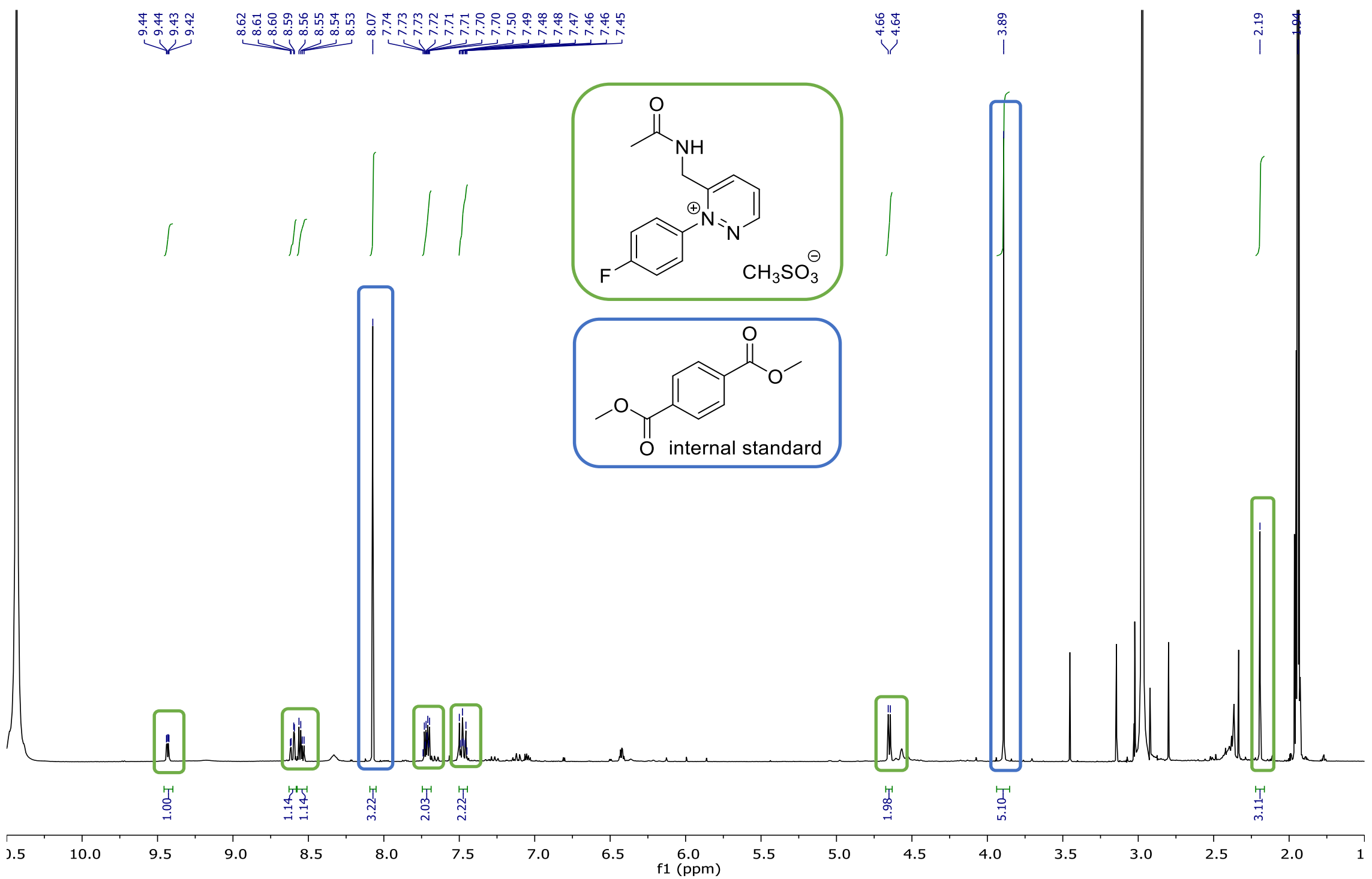


1-(4-Methoxyphenyl)-3,6-dimethylpyridazin-1-ium methanesulfonate (19') DEPTQ-NMR (CD $\left.{ }_{3} \mathrm{CN}, 100 \mathrm{MHz}\right)$

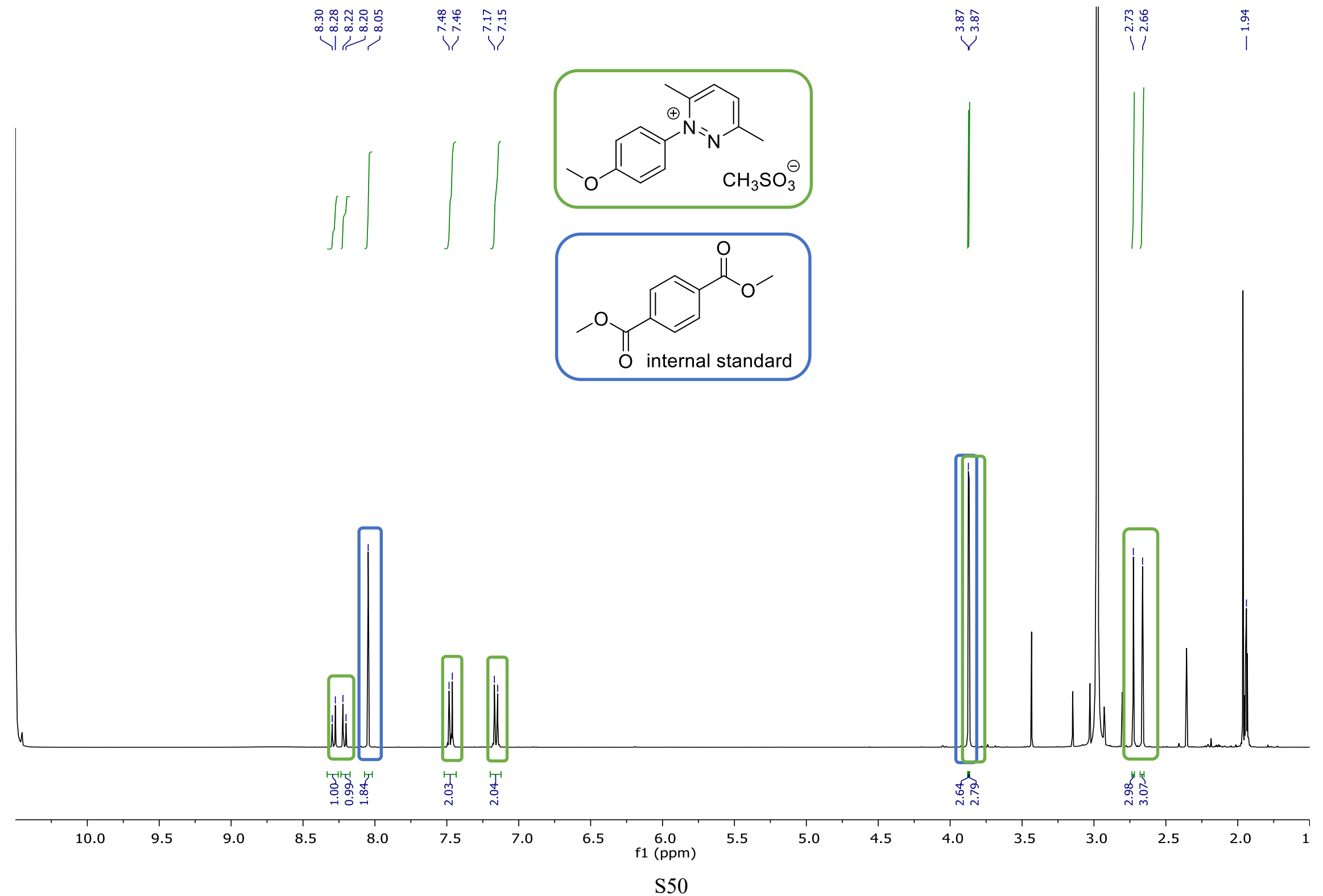


1-(4-Methoxyphenyl)-6-methylpyridazin-1-ium methanesulfonate (1 h') ${ }^{1} \mathrm{H}-\mathrm{NMR}$ (CD3CN, $\left.400 \mathrm{MHz}\right)$

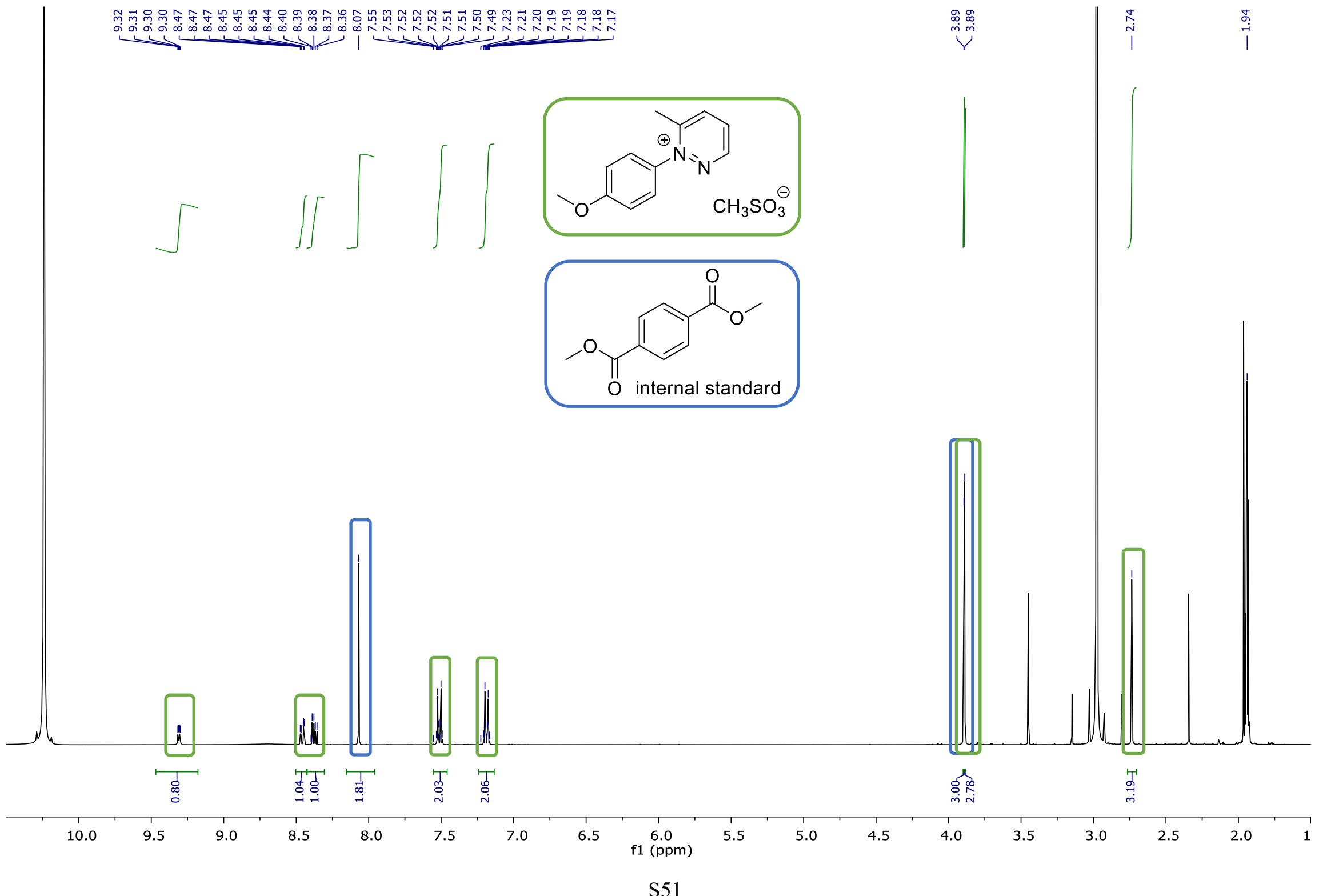


6-(Acetamidomethyl)-1-(4-methoxyphenyl)pyridazin-1-ium methanesulfonate (1i') ${ }^{1} \mathrm{H}-\mathrm{NMR}\left(\mathrm{CD}_{3} \mathrm{CN}, 400 \mathrm{MHz}\right)$

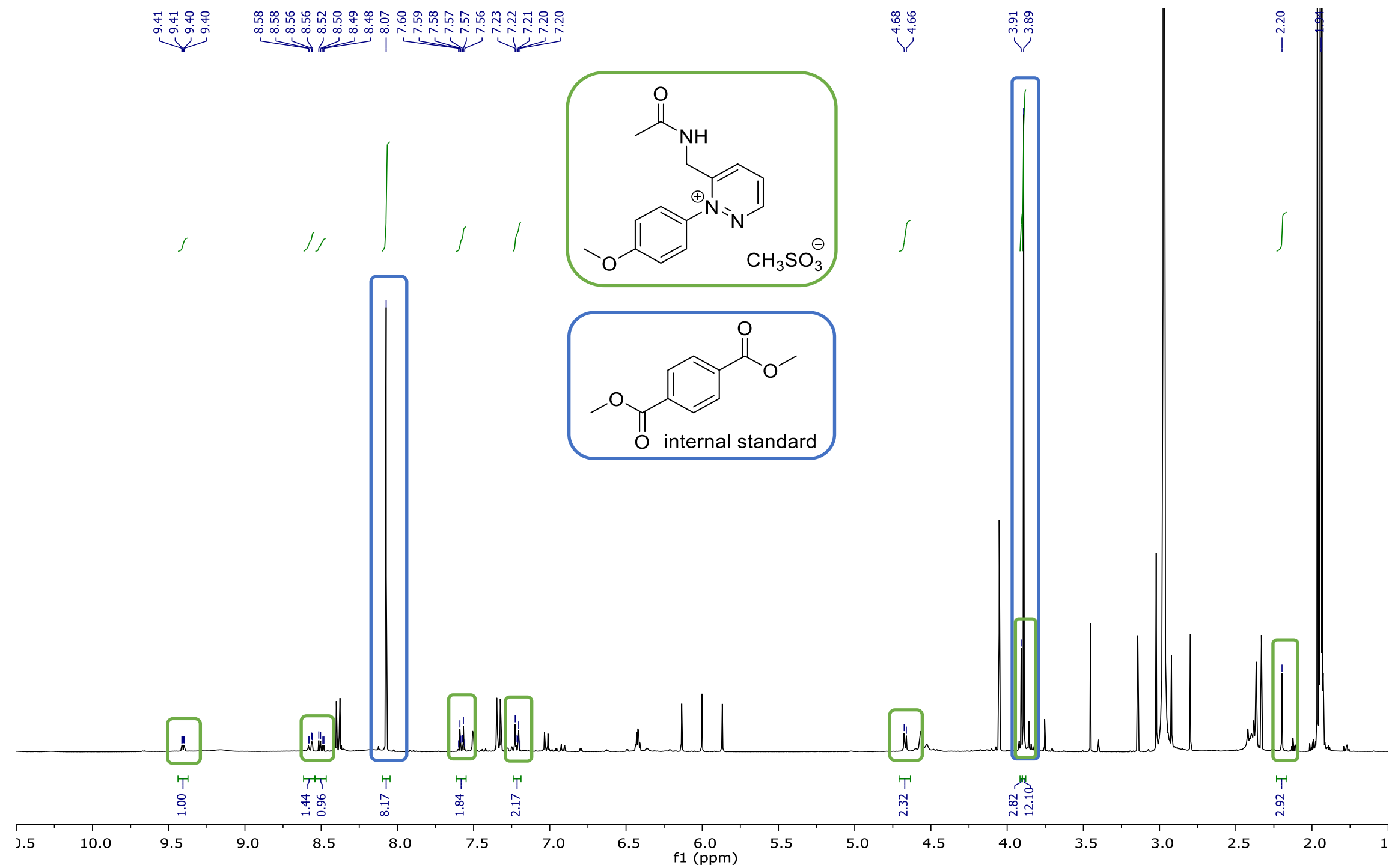


1-(4-Cyanophenyl)-3,6-dimethylpyridazin-1-ium methanesulfonate (1j') ${ }^{1} \mathrm{H}-\mathrm{NMR}\left(\mathrm{CD}_{3} \mathrm{CN}, 400 \mathrm{MHz}\right)$

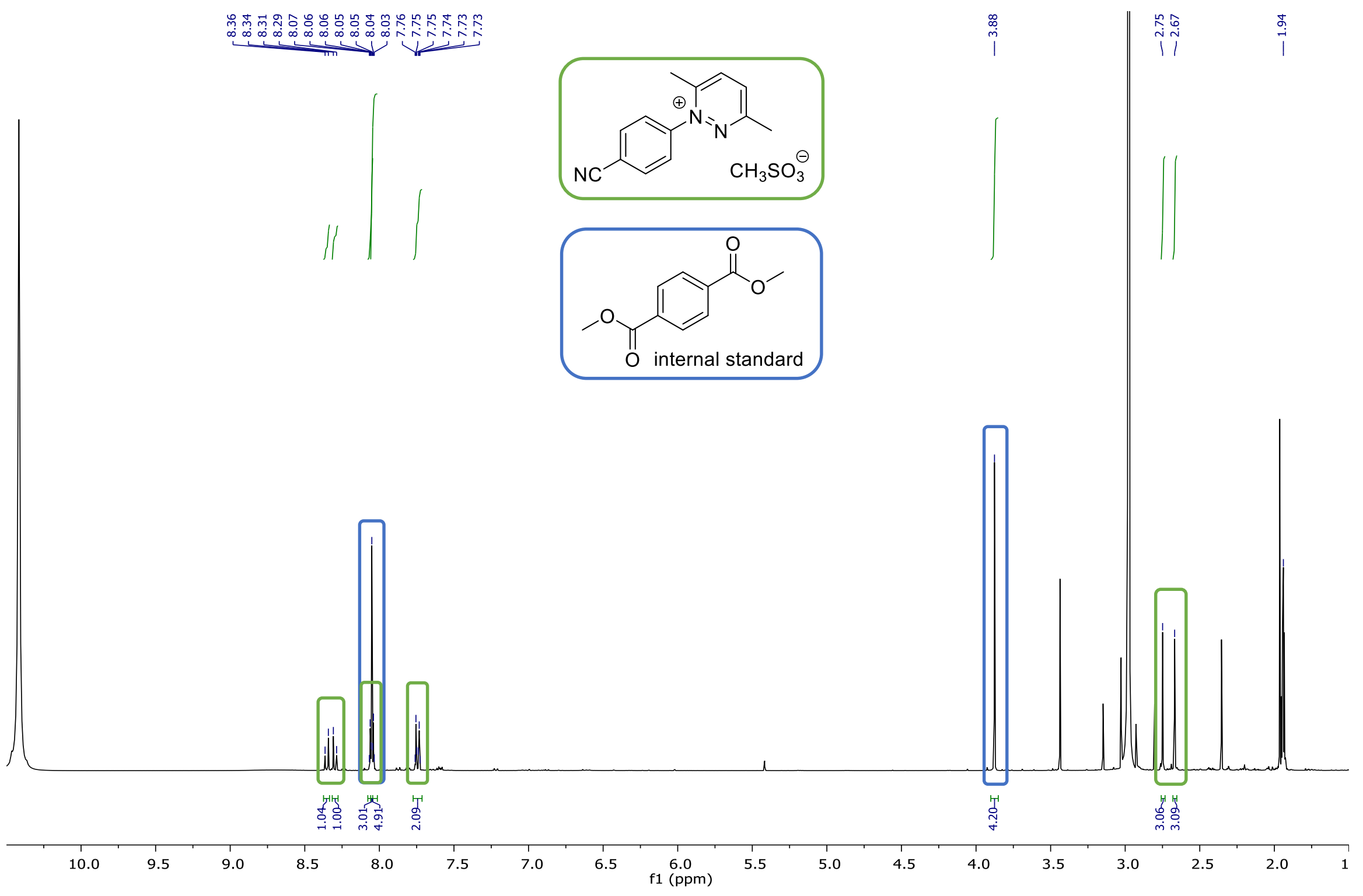




\section{1-(4-Cyanophenyl)-6-methylpyridazin-1-ium methanesulfonate (1k') ${ }^{1} \mathrm{H}-\mathrm{NMR}\left(\mathrm{CD}_{3} \mathrm{CN}, 400 \mathrm{MHz}\right)$}

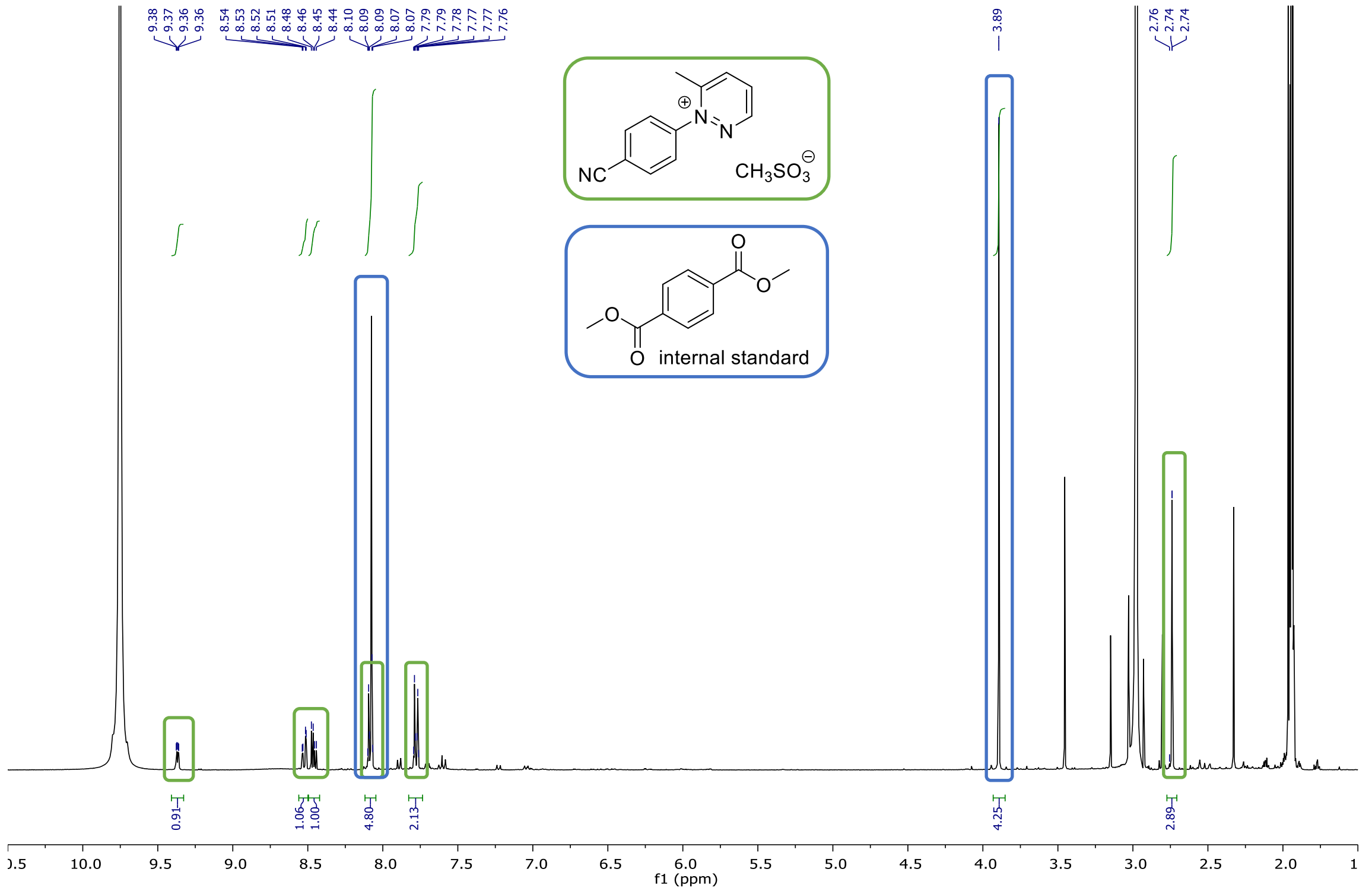


6-(Acetamidomethyl)-1-(4-methoxyphenyl)pyridazin-1-ium methanesulfonate (11') ${ }^{1} \mathrm{H}-\mathrm{NMR}\left(\mathrm{CD}_{3} \mathrm{CN}, 400 \mathrm{MHz}\right)$

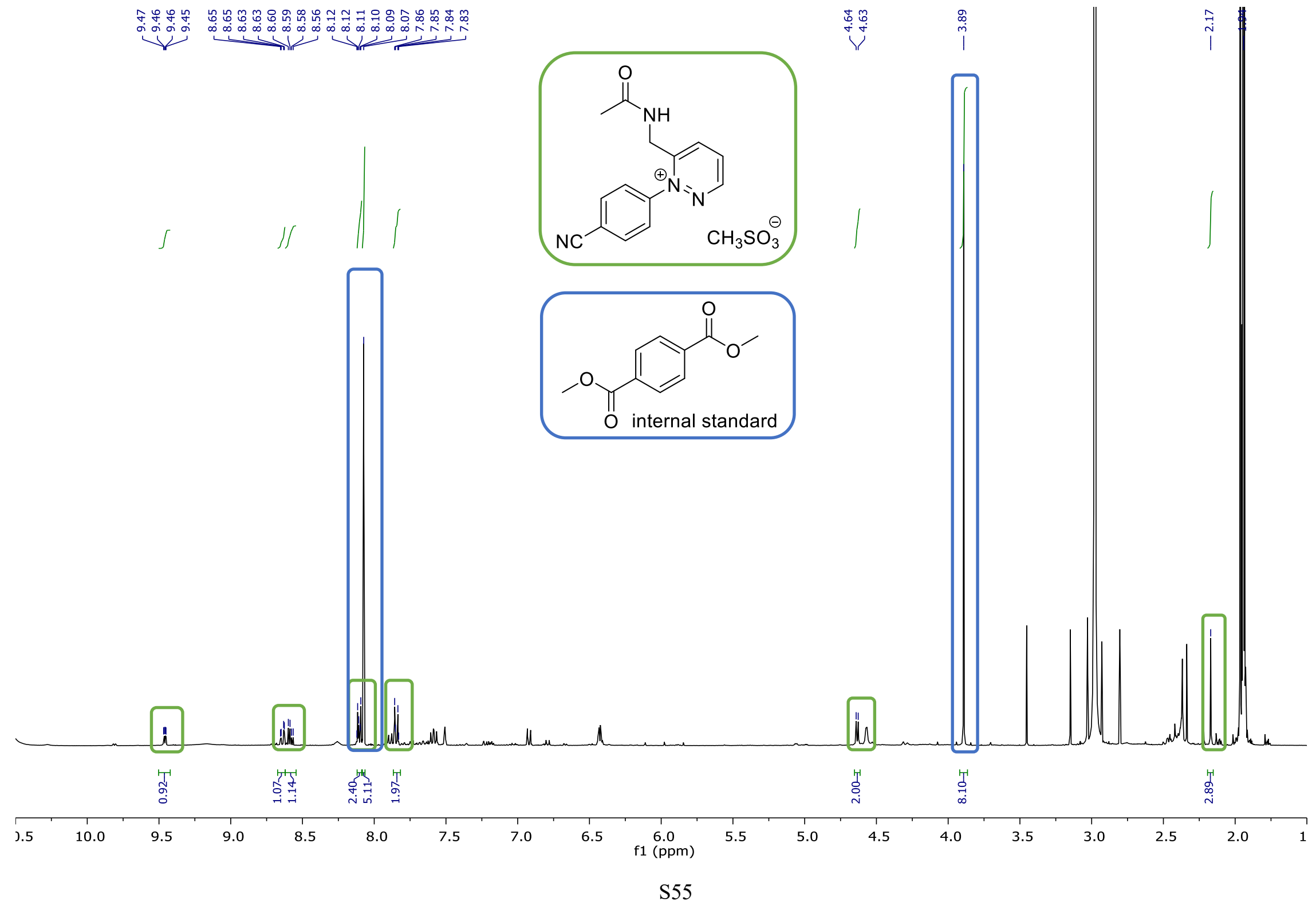




\section{Spectra of tetrahydropyridazines $(10 \mathrm{a}, \mathrm{d}, \mathrm{g})$}

1-(4-Chlorophenyl)-3,6-dimethyl-1,4,5,6-tetrahydropyridazine (10a) ${ }^{1} \mathrm{H}-\mathrm{NMR}\left(\mathrm{CDCl}_{3}, 400 \mathrm{MHz}\right)$

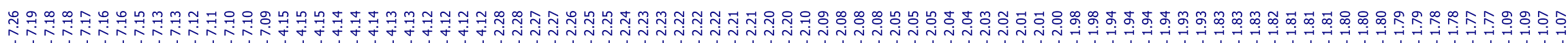
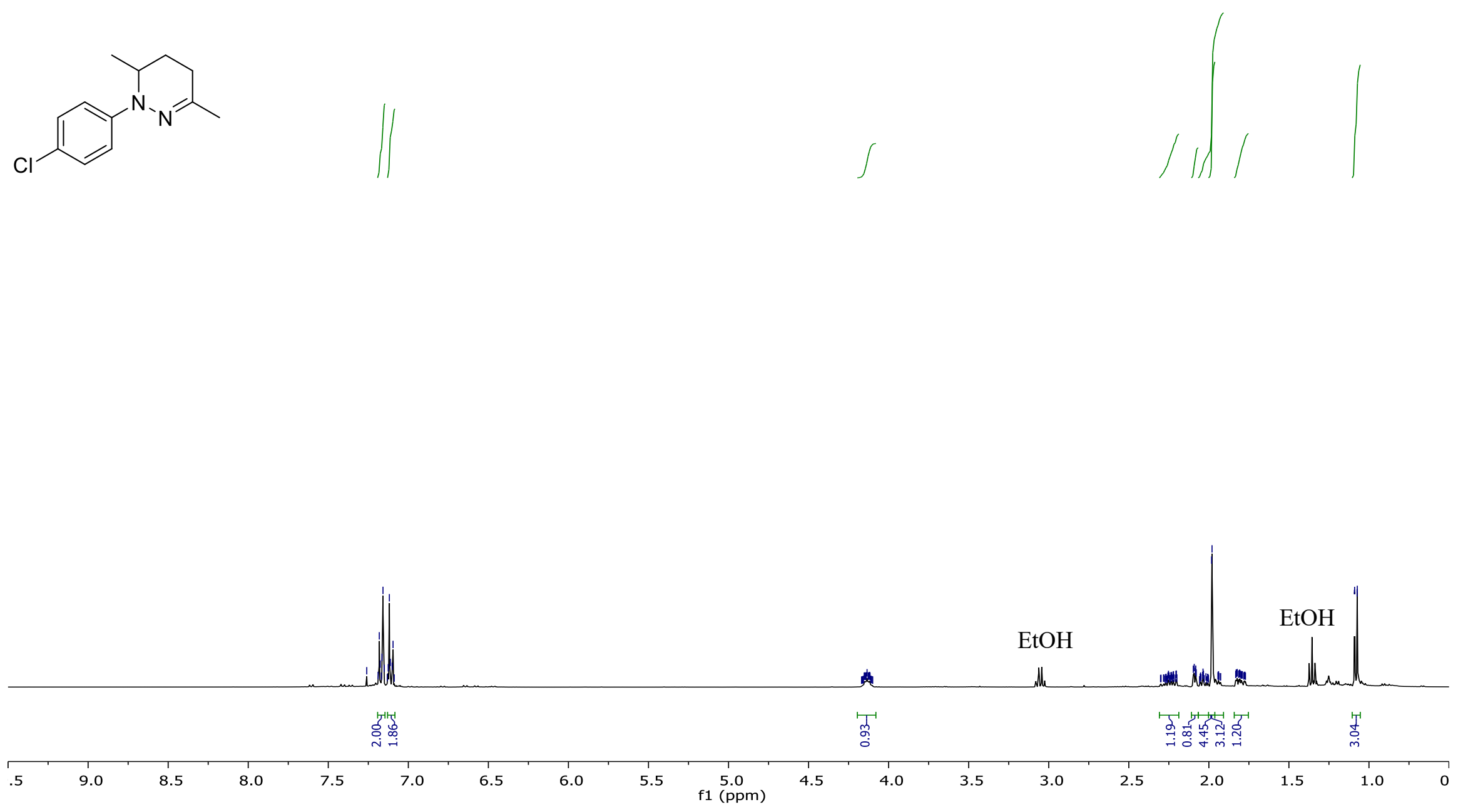
1-(4-Chlorophenyl)-3,6-dimethyl-1,4,5,6-tetrahydropyridazine (10a) DEPTQ-NMR $\left(\mathrm{CD}_{3} \mathrm{CN}, 100 \mathrm{MHz}\right)$
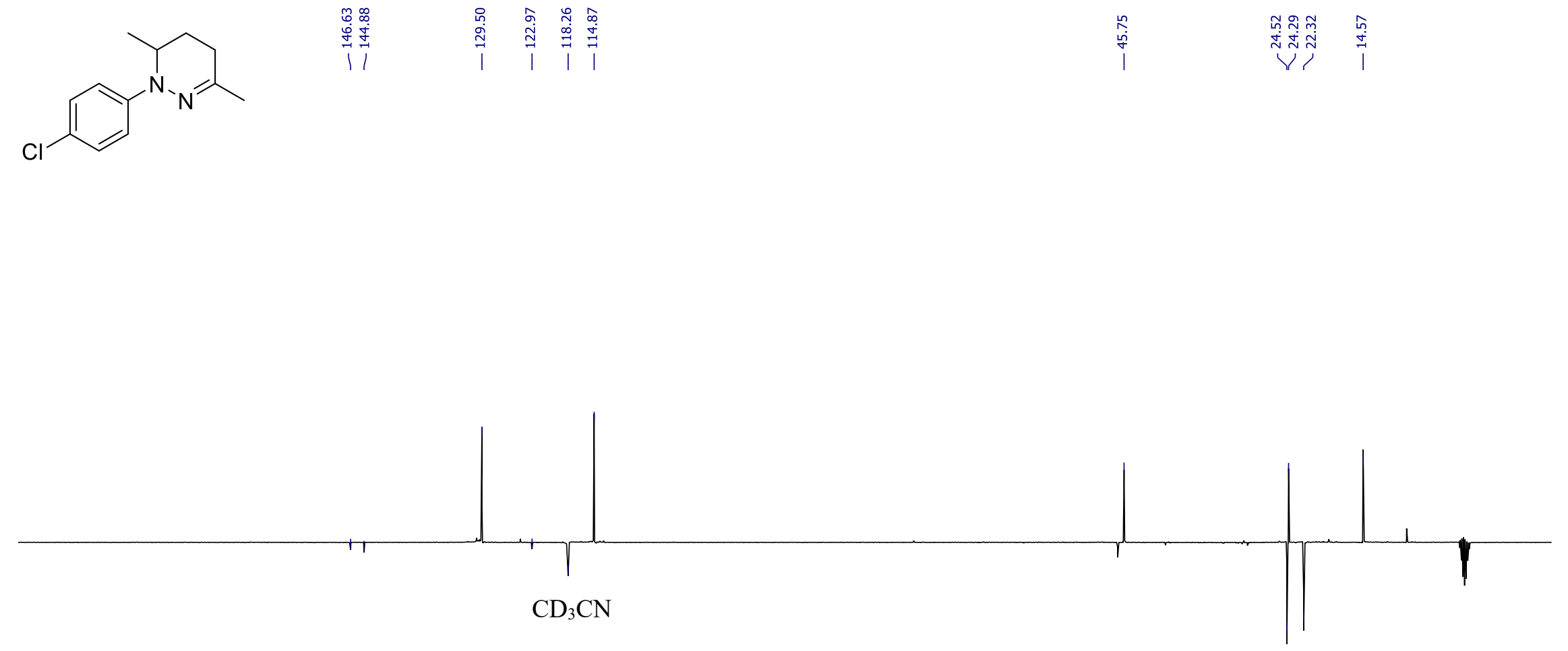

\begin{tabular}{|c|c|c|c|c|c|c|c|c|c|c|c|c|c|c|c|c|c|c|c|}
\hline 90 & 180 & 170 & 160 & 150 & 140 & 130 & 120 & 110 & 100 & $\begin{array}{c}90 \\
\mathrm{f} 1(\mathrm{ppm})\end{array}$ & 80 & 70 & 60 & 50 & 40 & 30 & 20 & 10 & 0 \\
\hline
\end{tabular}




\section{1-(4-Fluorophenyl)-3,6-dimethyl-1,4,5,6-tetrahydropyridazine (10d) ${ }^{1} \mathrm{H}-\mathrm{NMR}\left(\mathrm{CDCl}_{3}, 400 \mathrm{MHz}\right)$}

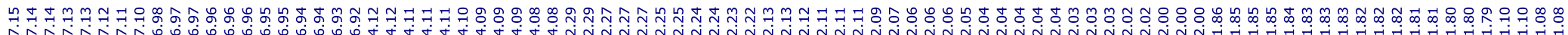
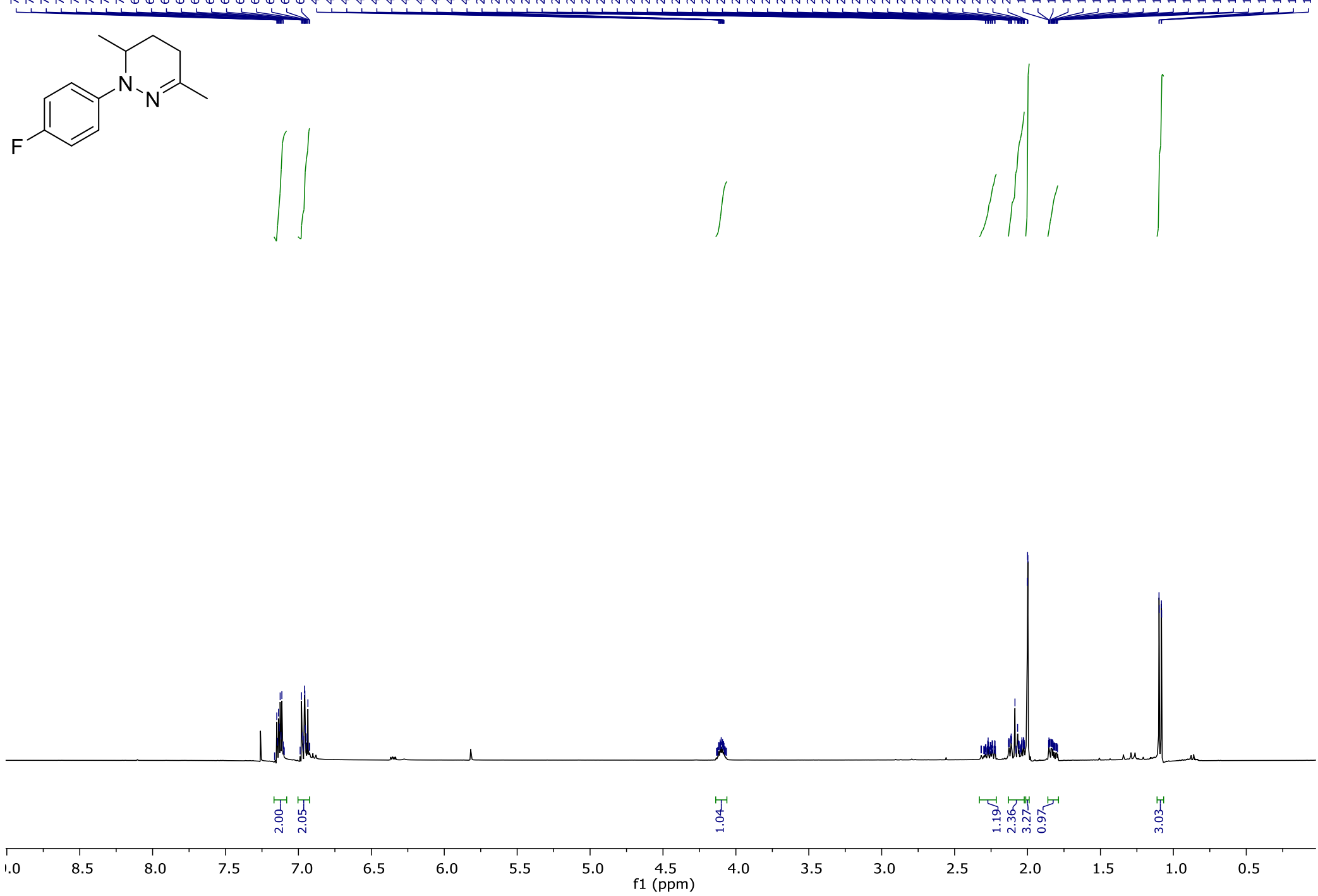
1-(4-Fluorophenyl)-3,6-dimethyl-1,4,5,6-tetrahydropyridazine (10d) DEPTQ-NMR $\left(\mathrm{CD}_{3} \mathrm{CN}, 100 \mathrm{MHz}\right)$

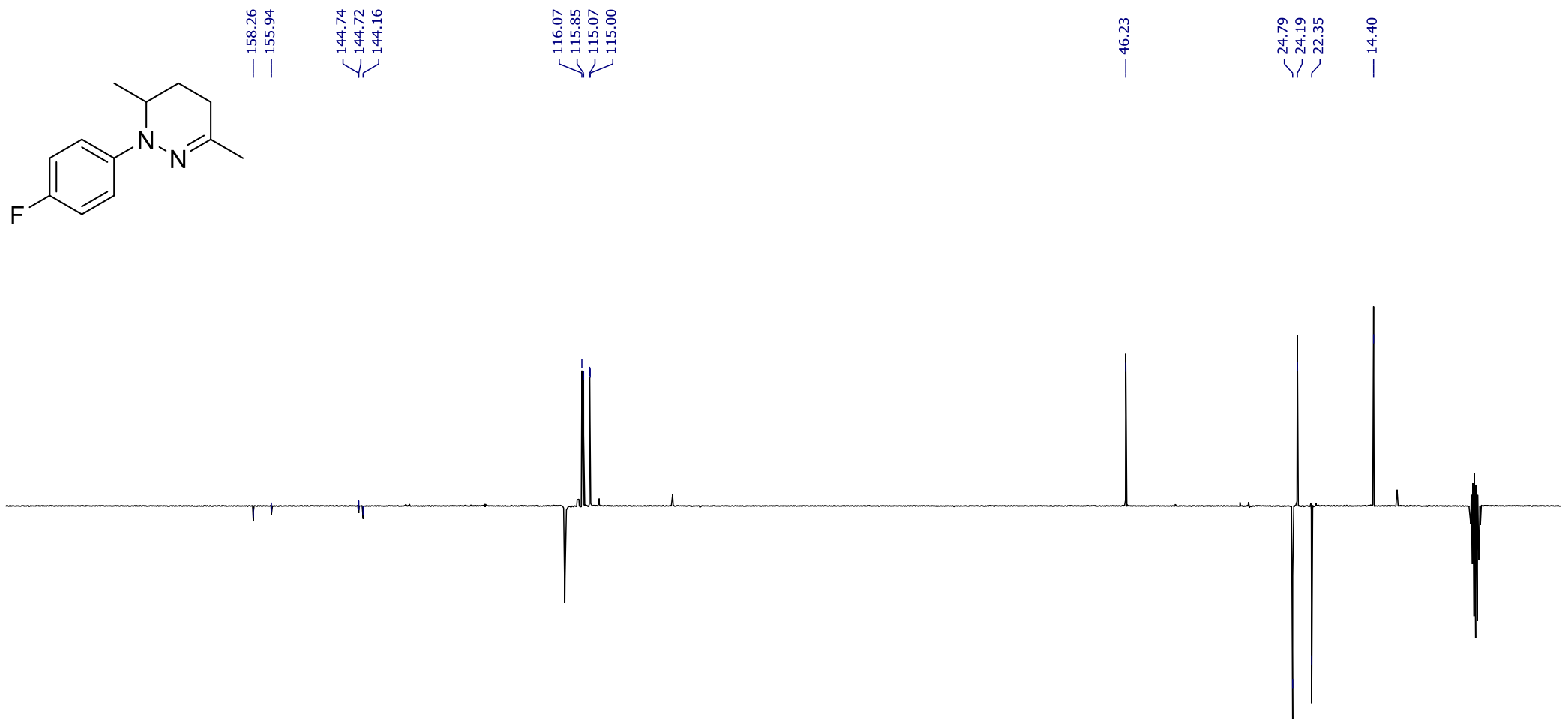


1-(4-Methoxyphenyl)-3,6-dimethyl-1,4,5,6-tetrahydropyridazine (10g) ${ }^{1} \mathrm{H}-\mathrm{NMR}\left(\mathrm{CDCl}_{3}, 400 \mathrm{MHz}\right)$

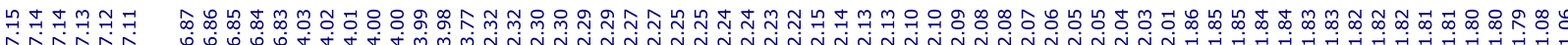<smiles>COc1ccc(N2N=C(C)CCC2C)cc1</smiles>
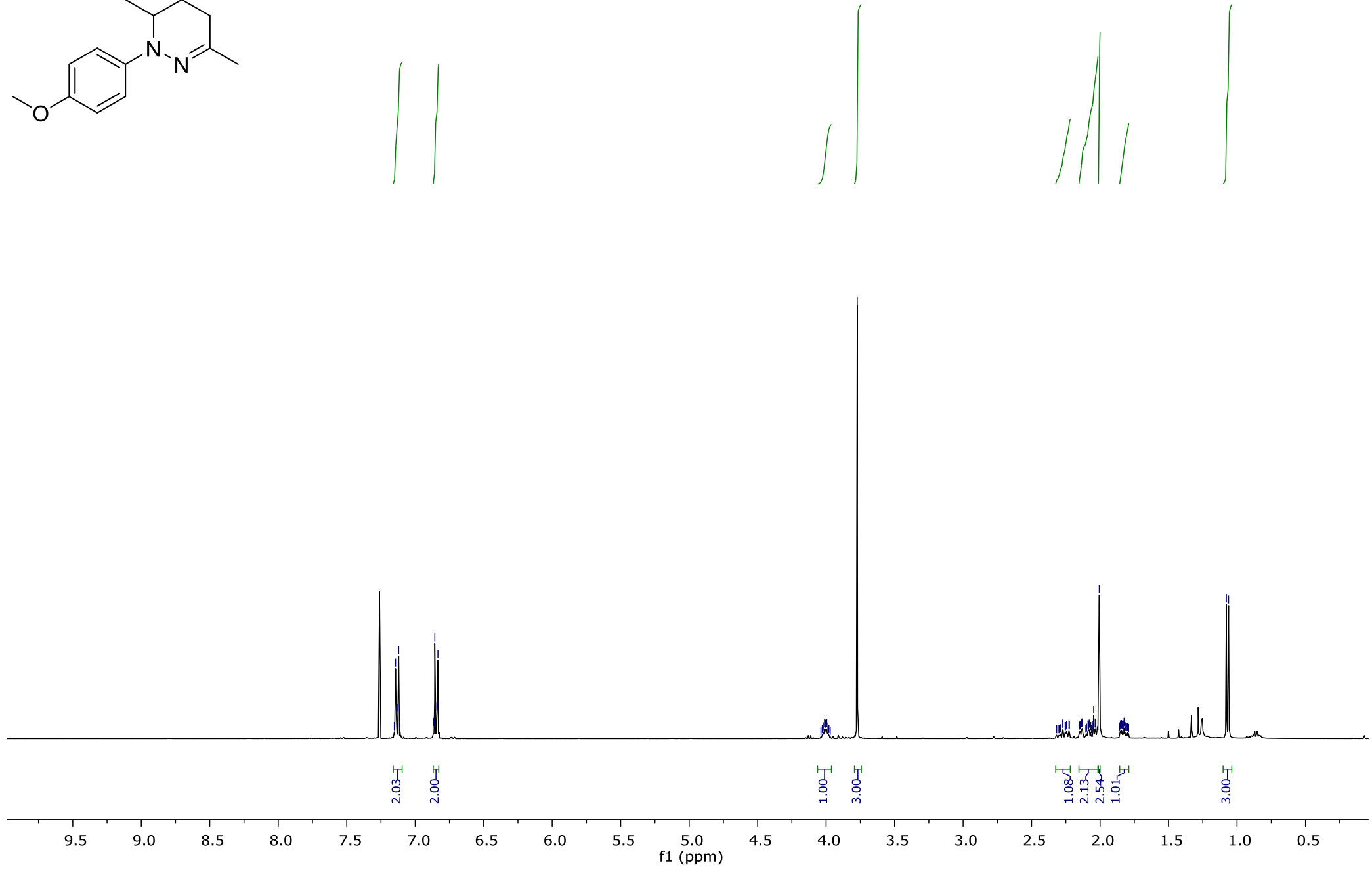
1-(4-Methoxyphenyl)-3,6-dimethyl-1,4,5,6-tetrahydropyridazine (10g) DEPTQ-NMR $\left(\mathrm{CD}_{3} \mathrm{CN}, 100 \mathrm{MHz}\right)$

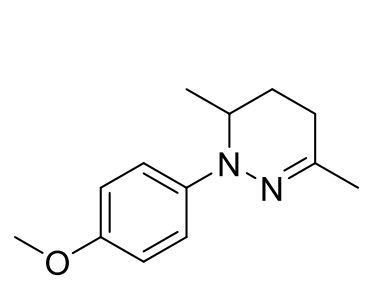

$$
\text { 要题 }
$$

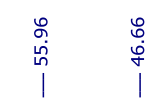

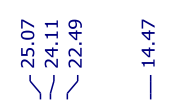

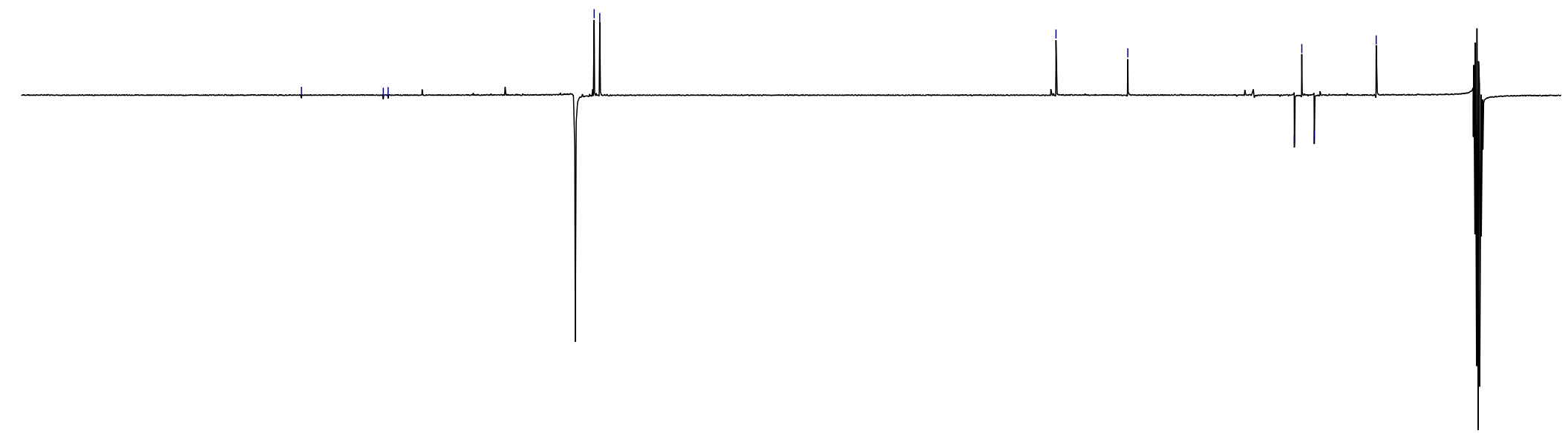


5. Spectra of azo compound (14)

1-(tert-butyl)-2-(4-chlorophenyl)diazene (14) ${ }^{1} \mathrm{H}-\mathrm{NMR}\left(\mathrm{CDCl}_{3}, 400 \mathrm{MHz}\right)$

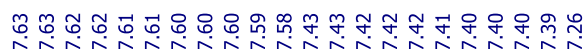

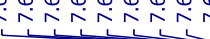

$\int$
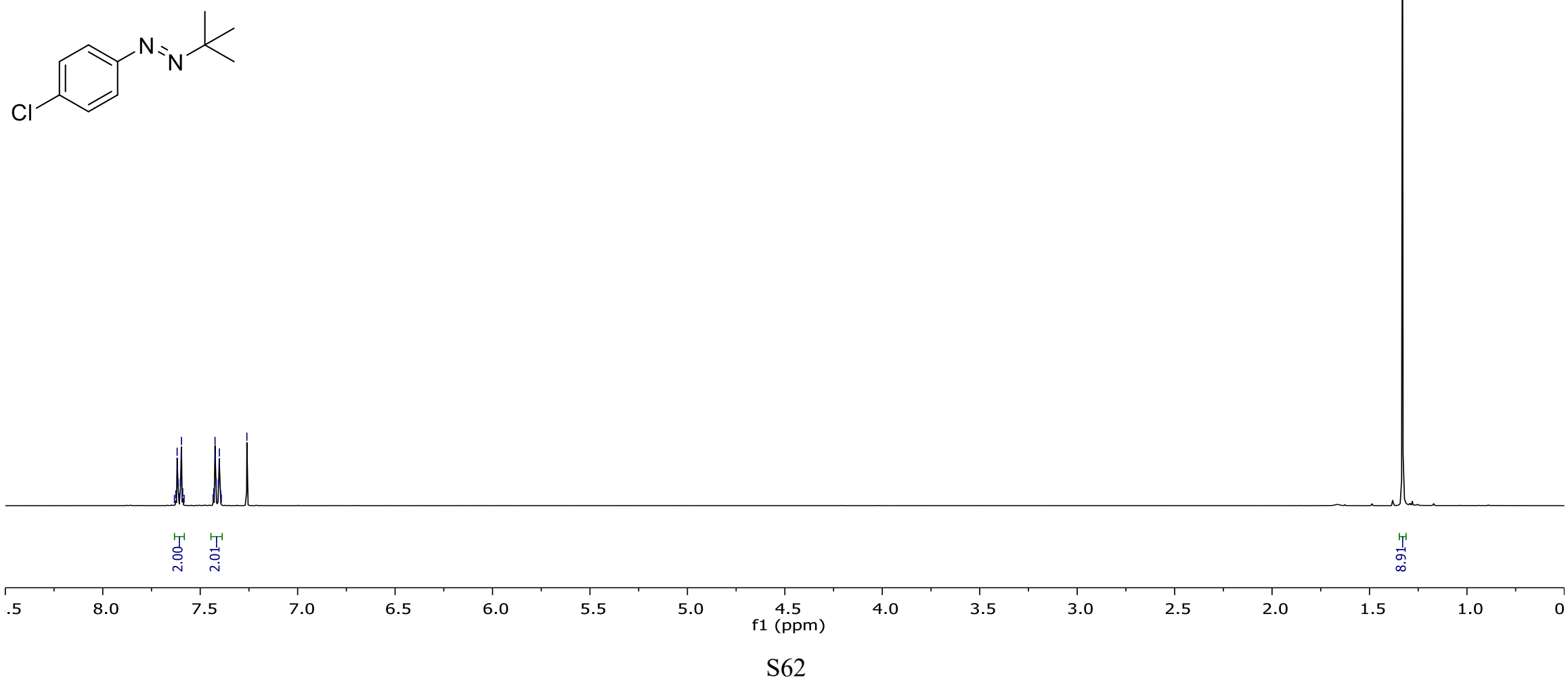
1-(tert-butyl)-2-(4-chlorophenyl)diazene (14) DEPTQ-NMR ( $\left.\mathrm{CDCl}_{3}, 100 \mathrm{MHz}\right)$

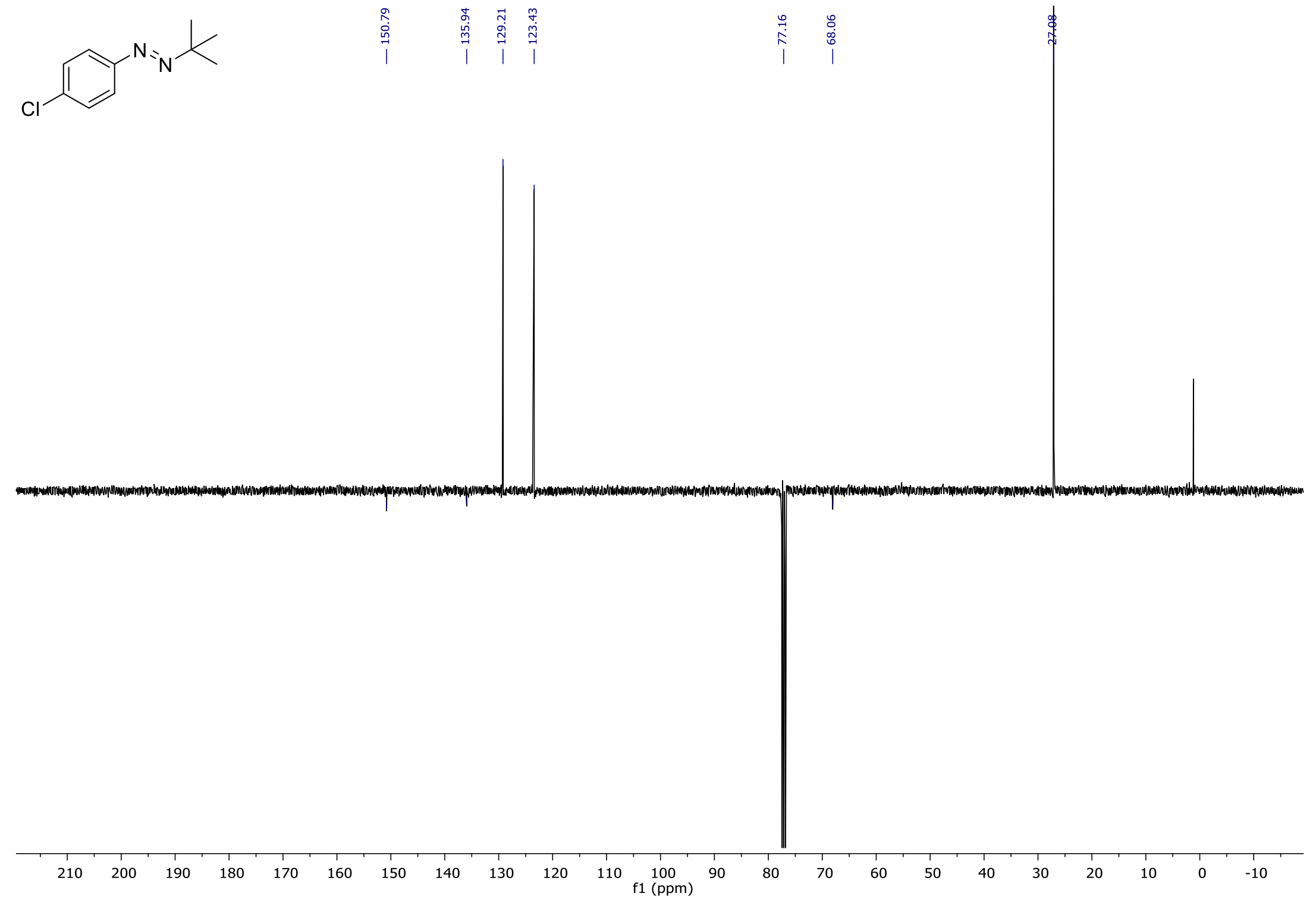

